\title{
Estudo de Métodos Estocásticos para Otimização Global de Problemas de Programação Não Linear
}

\author{
Érico Murilo Gozzi \\ DisSERTAÇÃO APRESENTADA \\ $\mathrm{AO}$ \\ InstituTo DE MATEMÁtica E Estatística \\ DA \\ Universidade DE SÃo Paulo \\ PARA \\ OBTENÇÃO DO TÍTULO DE MESTRE \\ EM \\ CIÊNCIAS \\ Área de Concentração: Ciência da Computação \\ Orientador: Prof. Dr. Ernesto G. Birgin
}

São Paulo, janeiro de 2007. 


\section{Estudo de Métodos Estocásticos para Otimização Global de Problemas de Programação Não Linear}

Este exemplar corresponde à redação final da dissertação de mestrado devidamente corrigida e defendida por Érico Murilo Gozzi e aprovada pela comissão julgadora.

Banca examinadora:

- Prof. Dr. Ernesto G. Birgin (Orientador) - IME-USP

- Prof. Dr. Marcelo Gomes de Queiroz - IME-USP

- Prof. Dr. Roberto Andreani - IMECC-UNICAMP 


\section{Sumário}

1 Introdução 4

2 O algoritmo Random Linkage 7

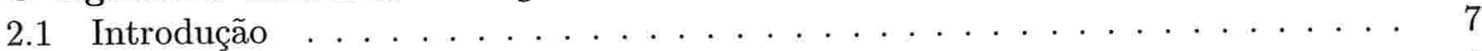

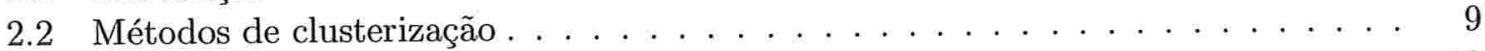

$2.3 \mathrm{O}$ algoritmo Random Linkage . . . . . . . . . . . . . . . . . . . . 13

3 Utilização da curva de Lissajous como estratégia de Tunneling 15

3.1 Introdução . . . . . . . . . . . . . . . . . . . . 15

3.2 A curva de Lissajous . . . . . . . . . . . . . . . . . . . . . . . 18

3.2.1 Exemplo da utilização da curva de Lissajous . . . . . . . . . . . . . . 20

4 O algoritmo GRASP 21

4.1 Introdução . . . . . . . . . . . . . . . . . . . . 21

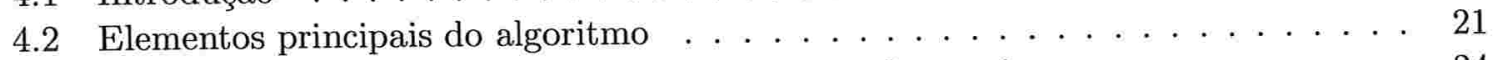

4.3 Aplicação do GRASP em problemas de otimização contínua . . . . . . . . . . . . . 24

4.3 .1 Fase de construção . . . . . . . . . . . . . . . . . . . . . . . . . . 26

4.3 .2 Fase de busca local . . . . . . . . . . . . . . . . . . . . . 27

4.4 GRASP Contínuo . . . . . . . . . . . . . . . . . . 28

5 Experimentos computacionais 32

5.1 Introdução . . . . . . . . . . . . . . . . . . . . . . . 32

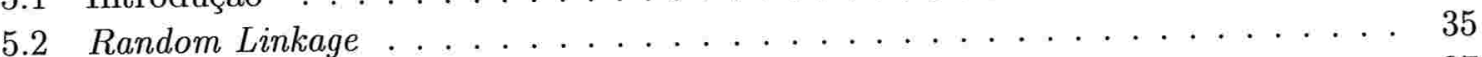

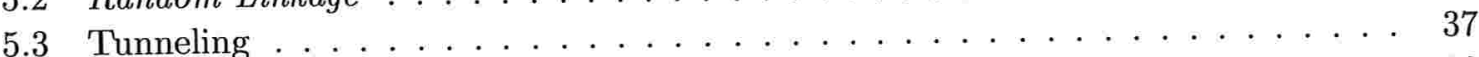

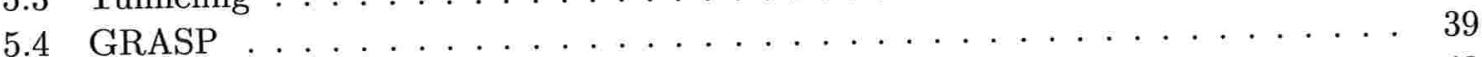

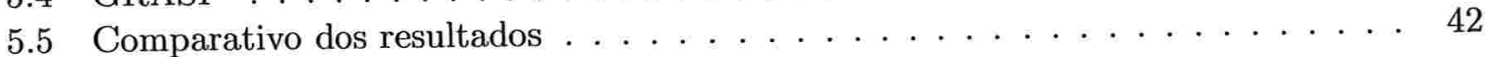

6 Experimentos com problemas de empacotamento 45

6.1 Introdução . . . . . . . . . . . . . . . . . . . . 45

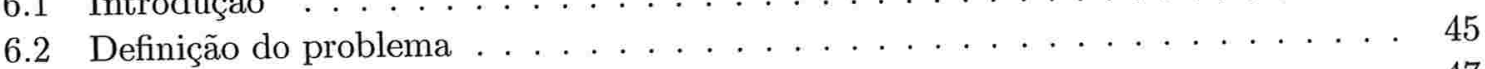

6.3 Multistart . . . . . . . . . . . . . . . . . . . . . . . . 47

6.4 Random Linkage . . . . . . . . . . . . . . . . . . . . . . . . . . . . . . . . . . . . .

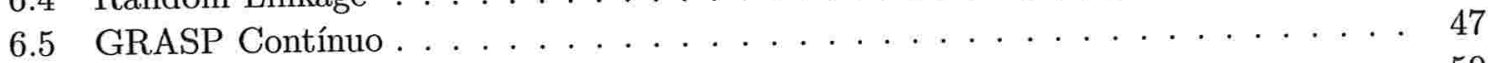

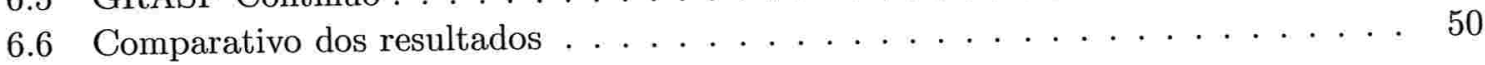


Apêndices $\quad 62$

A Funções utilizadas nos experimentos 162

B Fluxogramas dos algoritmos implementados $\quad 68$

B.1 Multistart . . . . . . . . . . . . . . . . . . . . . 68 68

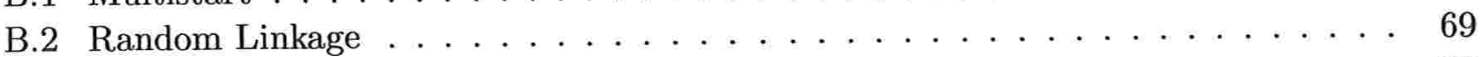

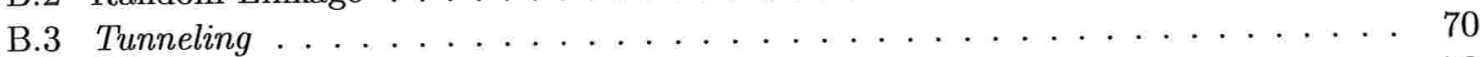

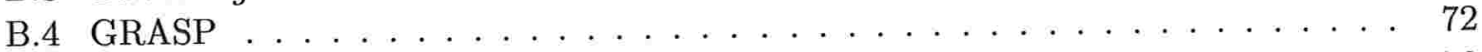

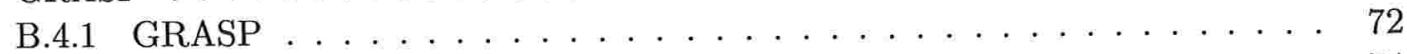

B.4.2 GRASP contínuo . . . . . . . . . . . . . . . 74

C Comparação alternativa dos testes realizados no Capítulo 5 77

C.1 Comparativo dos resultados . . . . . . . . . . . . . . . . . . 77

$\begin{array}{lr}\text { Bibliografia } & \mathbf{8 0}\end{array}$ 


\section{Resumo}

O estudo de técnicas de otimização global encontra aplicação em uma grande diversidade de problemas, tais como: economia de escala, finanças, análise de risco, estatística, redes e problemas de transporte, banco de dados, biologia molecular, genética. No entanto, apesar de mais de quatro décadas de pesquisas nesta área, as soluções conhecidas para problemas de otimização global são aplicáveis, de forma eficiente, apenas a um conjunto restrito de problemas.

Os algoritmos de otimização global podem ser divididos em dois grandes grupos: determinísticos e estocásticos. Os métodos determinísticos garantem alcançar uma boa aproximação de um minimizador global em um número finito de passos; para os métodos estocásticos a probabilidade de encontrar um minimizador global tende a 1 quando o número de iterações tende a infinito.

O objetivo deste trabalho foi estudar três algoritmos estocásticos de otimização global: Random Linkage, Tunneling utilizando a curva de Lissajous e o GRASP adaptado para problemas de programação não linear. Para isso, implementamos os três métodos e realizamos experimentações com problemas clássicos de programação não linear. Em seguida, adaptamos os métodos para utilizar o algoritmo GENCAN como método de minimização local. Desta forma foi possível estabelecer um comparativo da eficácia de cada um dos métodos em escapar de minimizadores locais. 


\section{Abstract}

The study of global optimization techniques finds application in a range sort of problems, such that: scale economy, finances, risk analysis, statistics, network and transportation problems, databases, molecular biology, genetics. However, in despite of more than four decades of research in this area, the known solutions for global optimization problems are applicable, in a efficient form, only to a few set of problems.

Global optimization algorithms can be divided in two major groups: deterministics and stochastics. The determistics methods guarantee to achieve a good approximation of the global optimizer in a finite number of steps; in the stochastics methods the probability of finding a good approximation of the global optimizer tends to 1 (one) when the number of steps tends to infinity.

The objective of this work was to study three stochastics global optimization algorithms: Random Linkage, Tunneling using the Lissajous curve and GRASP adapted for solving nonlinear optimization problems. We implemented the three methods and realized several computational experiments with classic nonlinear problems. Then, we adapted the methods to use GENCAN as the local minimization method. In such a way it was possible to compare the effectiveness of each method in scape of local minimizers. 


\section{Capítulo 1}

\section{Introdução}

O estudo de técnicas de otimização global encontra aplicação em uma grande diversidade de problemas, tais como: economia de escala, finanças, análise de risco, estatística, redes e problemas de transporte, banco de dados, biologia molecular, genética. No entanto, apesar de mais de quatro décadas de pesquisas nesta área, as soluções conhecidas para problemas de otimização global são aplicáveis, de forma eficiente, apenas a um conjunto restrito de problemas.

O problema considerado neste trabalho consiste na minimização de uma função $f: \Re^{n} \rightarrow \Re$ suave e com limitantes nas variáveis. O conjunto factível $\Omega$ é definido por $\Omega=\left\{x \in \Re^{n} \mid l \leq\right.$ $x \leq u\}$. Um ponto $x^{*}$ é uma solução global para o problema em questão, se e somente se, $f\left(x^{*}\right) \leq f(x) \forall x \in \Omega$. Este ponto é chamado de minimizador global de $f$, sendo $f\left(x^{*}\right)$ o mínimo global do problema.

Uma das primeiras estratégias sugeridas para a resolução de problemas de otimização global [9] consistiu em localizar e comparar o maior número possível de minimizadores locais do problema. Desta estratégia surgiu o método conhecido como Multistart onde, a partir de pontos iniciais aleatórios, eram utilizados métodos de minimização local visando descobrir o maior número possível de soluções locais. Com isto, este método atinge melhores aproximações do minimizador global a medida em que novas soluções locais são descobertas.

Uma outra estratégia contemporânea ao Multistart surgiu do princípio de que a aproximação do minimizador global não precisaria ser um minimizador local. Seguindo esta prerrogativa foi criado um método de "força bruta" conhecido como Busca em Malha (Grid search method) que consiste em mapear $\Omega$ em pontos equidistantes utilizando um passo fixo $h$. A partir deste mapeamento, o ponto com o menor valor de função é devolvido pelo algoritmo como sendo a aproximação da solução global do problema.

Destes dois exemplos é possível dividir os métodos de otimização global em dois grandes grupos $[9,21]$ :

- Estocásticos, cuja probabilidade de convergirem para uma solução global tende a 1 quando o número de passos do algoritmo tende a infinito, como no caso do Multistart.

- Determinísticos, que garantem alcançar uma $\epsilon$-vizinhança de uma solução global em um 
número finito de passos, como no caso da Busca em Malha.

A medida em que há um crescimento na complexidade e no porte dos problemas a serem resolvidos, o uso de algoritmos determinísticos torna-se proibitivo devido a quantidade de processamento necessário. Salvo casos de algoritmos determinísticos específicos, tais como os utilizados em programação côncava e métodos DC (desigualdade entre duas funções convexas), a melhor estratégia a ser seguida é a utilização de métodos estocásticos.

Os algoritmos estocásticos procuram, a cada passo, melhorar a sua aproximação da solução ótima de forma que, em um número infinito de passos, a probabilidade de encontrarem uma boa aproximação do ótimo global tende a 1.

Dentre as diversas estratégias utilizadas pelos Métodos Estocásticos, destacamos os Métodos de Duas Fases [7] que consistem em adaptar os métodos de otimização local para resolverem problemas de otimização global. Estes métodos são compostos por uma mistura de duas etapas:

1. Etapa de Exploração, ou Fase Global, cujo objetivo é encontrar pontos em diferentes regiões de $\Omega$ na tentativa de cobrir as diversas regiões de atração dos minimizadores locais do problema de otimização.

2. Etapa de Refinamento, ou Fase Local, onde é realizada uma exploração local em busca de uma boa aproximação do minimizador global do problema.

Após sucessivas execuções destas duas etapas, a melhor solução encontrada pela Etapa de Refinamento é considerada pelo algoritmo como a aproximação da solução ótima do problema proposto.

O grau de conhecimento dos elementos que compõem o problema a ser resolvido tem um impacto considerável nas estratégias seguidas pelos algoritmos. O objetivo deste trabalho é implementar e comparar três algoritmos estocásticos aplicados a problemas de programação não linear (PNL) com restrições de caixa. Os algoritmos apresentados nos próximos capítulos, trabalham com níveis diferentes de informação sobre a função objetivo e a região factível.

No Capítulo 2 descrevemos o método Random Linkage, apresentado em [19]. Esse método foi idealizado a partir de melhorias realizadas no algoritmo proposto em [24, 25], que utilizase da estratégia de clusterização da região factível com intuito de diminuir a quantidade de minimizações locais desnecessárias. Mostramos inicialmente o método original, proposto em [24, 25], e a seguir analisamos e implementamos as mudanças descritas em [19].

No Capítulo 3 detalhamos a técnica de Tunneling, descrita em [17, 16], e apresentamos uma implementação do algoritmo proposto em [23] que utiliza a curva de Lissajous na etapa de Tunneling. Descrevemos ainda a nossa implementação do algoritmo proposto em [23] e apresentamos a aplicação do mesmo em um problema de empacotamento de círculos.

No Capítulo 4 apresentamos o método GRASP aplicado a problemas de programação não linear com restrições de caixa. O GRASP, originalmente, proposto em [11, 12] para problemas de otimização combinatória, foi adaptado em [20] para problemas de otimização contínua e melhorado em [14, 15]. Para análise deste método, implementamos os algoritmos propostos em $[20]$ e $[14,15]$. 
No Capítulo 5 mostramos experimentos com os métodos descritos nos capítulos anteriores para um conjunto de 52 testes encontrados na literatura clássica de programação não linear. A partir dos resultados obtidos neste capítulo realizamos, no Capítulo 6, experimentos em problemas de empacotamento com os 3 métodos que obtiveram os melhores resultados nos testes gerais.

Concluímos este trabalho com uma análise geral do desempenho dos algoritmos considerando todos os experimentos realizados nos capítulos 5 e 6 . 


\section{Capítulo 2}

\section{O algoritmo Random Linkage}

\subsection{Introdução}

Os algoritmos estocásticos utilizados para a minimização de uma função $f$ qualquer, têm como característica comum encontrar uma boa aproximação do mínimo global com probabilidade 1 quando o número de iterações do método tende a infinito. Com o intuito de explorarmos melhor esta característica consideremos um procedimento cuja única ação seja gerar pontos aleatórios factíveis em $\Omega$ e que obedeçam a uma distribuição uniforme. Queremos que este procedimento encontre um ponto $y \in B_{\Omega}\left(\epsilon, x^{*}\right)$ tal que $B_{\Omega}\left(\epsilon, x^{*}\right)=\left\{y \in \Omega \mid\left\|y-x^{*}\right\| \leq \epsilon\right.$, para $\left.\epsilon>0\right\}$, onde $x^{*} \in \Omega$ é um minimizador global.

A probabilidade de que até a $K$-ésima iteração tenha sido encontrado pelo menos um ponto em $B_{\Omega}\left(\epsilon, x^{*}\right)$ é

$$
\begin{gathered}
\operatorname{Pr}\left(x_{k} \in B_{\Omega}\left(\epsilon, x^{*}\right) \text { para } 1 \leq k \leq K\right)= \\
1-\operatorname{Pr}\left(x_{1}, \ldots, x_{K} \notin B_{\Omega}\left(\epsilon, x^{*}\right)\right)=1-\left(1-\varphi\left(B_{\Omega}\left(\epsilon, x^{*}\right)\right)\right)^{K}
\end{gathered}
$$

onde $\varphi\left(B_{\Omega}\left(\epsilon, x^{*}\right)\right)$ corresponde à probabilidade do ponto ser sorteado nas regiões de $\Omega$. Como $\varphi\left(B_{\Omega}\left(\epsilon, x^{*}\right)\right)>0$, então a probabilidade acima tende a 1 quando $K$ tende a $\infty$. Portanto, o procedimento acima apresenta características de um Método Estocástico.

Deste exemplo, deriva o algoritmo de otimização global conhecido como Pure Random Search, que utiliza-se apenas da Etapa de Exploração para a geração das possíveis soluções do problema de otimização.
Algoritmo 2.1. Pure Random Search
Dado $K \in \mathbb{N}$
Passo 0. $f_{\min } \leftarrow \infty ; k \leftarrow 1$
Passo 1. Gere um ponto $x_{k} \in \Omega$ com distribuição uniforme
Se $f\left(x_{k}\right)<f_{\min }$ então 


$$
\begin{aligned}
& f_{\min } \leftarrow f\left(x_{k}\right) \\
& y \leftarrow x_{k}
\end{aligned}
$$

fim se

Passo 2. $k \leftarrow k+1$

Se $k<K$ volte ao Passo 1

O ponto $y$ é retornado como aproximação de um minimizador global de $f$.

No entanto, este primeiro algoritmo não é considerado um Método de Duas Fases. Isto porque é formado apenas pela Etapa de Construção, onde são gerados os pontos $x_{k}$. Visando aumentar a eficiência do algoritmo foi incluída uma Fase de Refinamento composta por um procedimento de minimização local $P$ que, a partir de um ponto inicial $x_{k}$, retorna um minimizador local $\tilde{x}_{k}$ (referenciaremos a execução deste procedimento utilizando a seguinte notação: $\tilde{x}_{k} \leftarrow P\left(x_{k}\right)$ ). Este algoritmo ${ }^{1}$, conhecido como Multistart, encontra-se descrito a seguir:

\section{Algoritmo 2.2. Multistart}

Dado $K \in \mathbb{N}$

Passo 0. $f_{\min } \leftarrow \infty ; k \leftarrow 1$

Passo 1. Gere um ponto $x_{k} \in \Omega$ com distribuição uniforme

Passo 2. $\tilde{x}_{k} \leftarrow P\left(x_{k}\right)$

$$
\begin{gathered}
\text { Se } f\left(\tilde{x}_{k}\right)<f_{\min } \text { então } \\
f_{\min } \leftarrow f\left(\tilde{x}_{k}\right) \\
y \leftarrow \tilde{x}_{k}
\end{gathered}
$$

fim se

Passo 3. $k \leftarrow k+1$

\section{Se $k<K$ volte ao Passo 1}

O ponto $y$ é retornado como aproximação de um minimizador global de $f$.

Apesar deste método mostrar-se mais atrativo que o Pure Random Search, ainda assim apresenta baixa eficiência. Isto deve-se, principalmente, ao fato de que o mesmo minimizador local pode ser localizado várias vezes. Definindo a região de atração de um minimizador local $\tilde{x}$ como $R(\tilde{x})=\{x \in \Omega \mid \tilde{x} \leftarrow P(x)\}$, o ideal é que $P$ seja executado apenas uma vez para cada região de atração existente em $\Omega$. Visando resolver este problema foram propostas adaptações ao Multistart com a utilização de estratégias de clusterização da região factível.

\footnotetext{
${ }^{1}$ Os fluxogramas de todos os algoritmos que foram implementados e testados encontram-se descritos no Apêndice $\mathrm{B}$
} 


\subsection{Métodos de clusterização}

A idéia básica dos algoritmos de clusterização é particionar o conjunto factível $\Omega$ em diversas regiões de atração e executar $P$ uma única vez em cada uma destas regiões. A Figura (2.1) mostra uma comparação do Multistart com uma possível estratégia de clustering aplicados a função $f(x, y)=\sin (x)+\sin \left(\frac{10 x}{3}\right)+\log (x)-0.84 x+\sin (y)+\sin \left(\frac{10 y}{3}\right)+\log (y)-0.84 y$. Em (i) observamos, através das curvas de nível da função, a existência dos minimizadores locais $\left\{(3.4,3.4)^{t},(3.4,2.0)^{t},(2.0,2.0)^{t},(2.0,3.4)^{t}\right\}$. Em (ii), mostramos a aplicação do método Multistart a 500 pontos iniciais distintos, onde cada cor refere-se aos pontos iniciais que fizeram o método de minimização local convergir ao mesmo minimizador local. Em (iii) mostramos a aplicação de uma estratégia de clustering a estes mesmos 500 pontos iniciais, indicando com círculos coloridos os clusters que foram formados e a qual minimizador local convergiram. É válido ainda mencionar que, enquanto o Multistart realizou 500 minimizações locais, o método de clustering realizou apenas 16, descartando os demais pontos iniciais (referenciados em amarelo).

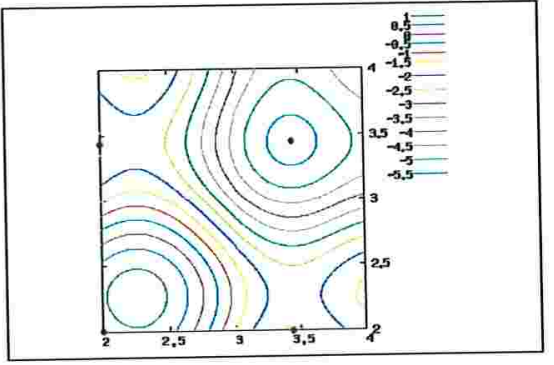

(i) Curvas de nivel

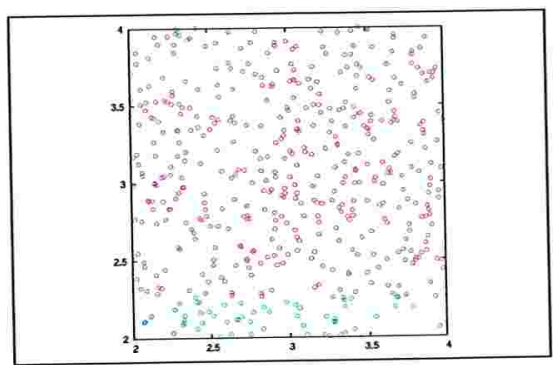

(ii) Multistart

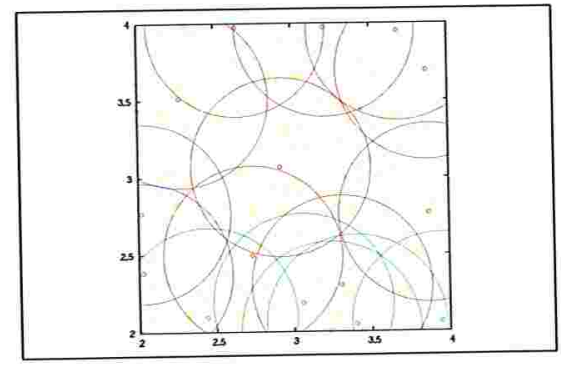

(iii) Estratégia de cluster

Figura 2.1: Comparação entre o Multistart e uma estratégia de clustering utilizando $f(x, y)=$ $\sin (x)+\sin \left(\frac{10 x}{3}\right)+\log (x)-0.84 x+\sin (y)+\sin \left(\frac{10 y}{3}\right)+\log (y)-0.84 y$ como função objetivo.

O algoritmo apresentado nesta seção, proposto em [24] com o nome de Density Clustering, restringe a execução do procedimento de minimização local $P$ para pontos muito próximos de um minimizador local.

O ponto de partida do método consiste em montar um conjunto $S$ de $N$ pontos aleatórios em $\Omega$, ordenados em ordem crescente de valor da função objetivo. Em seguida, para cada ponto $x_{i} \in S$, é verificado se existe no conjunto $\tilde{X}$ de minimizadores locais conhecidos um ponto $\tilde{x} \in \tilde{X}$ tal que a distância entre ambos é menor ou igual a uma distância crítica $r$. Caso não exista tal $\tilde{x}$, o procedimento $P$ é executado utilizando $x_{i}$ como ponto inicial.

Desta forma, a distância crítica $r$ é responsável pela definição de quais pontos se encontram na mesma região de atração $R(\tilde{x})$. Após todos os pontos de $S$ terem sido analisados, o algoritmo acrescenta $N$ novos pontos em $S$, reordenando $S$ em ordem crescente e repetindo todo o processo até que um número máximo de $K$ iterações seja atingido.

Visando proporcionar que os clusters alcancem, a cada nova iteração, um espaço maior da 
região factível, foi proposto em [24] a utilização do seguinte valor para a distância crítica $r$ em uma iteração $k$

$$
r_{k}=\pi^{-\frac{1}{2}}\left(k \Gamma\left(1+\frac{n}{2}\right) \mu(\Omega) \frac{\sigma \log k N}{k N}\right)^{\frac{1}{n}},
$$

onde $\Gamma(\cdot)$ corresponde à função $\operatorname{Gama}^{2}$ e $\mu(\cdot)$ a medida de Lebesgue.

O algoritmo descrito a seguir utiliza ainda uma constante $\gamma \in(0,1]$ para criar um subconjunto $S_{R} \subseteq S$. $S_{R}$ conterá os pontos em $S$ com menor valor da função objetivo.

\section{Algoritmo 2.3 Density Clustering}

Dados $K \in \mathbb{N}, N \in \mathbb{N}, \tilde{X}=\emptyset, S=\emptyset$ e $\gamma \in(0,1]$

Passo 0. $k \leftarrow 1$

Passo 1. Calcule $r_{k}$ de acordo com (2.1)

Passo 2. Inclua em $S N$ pontos aleatórios de $\Omega$ com distribuição uniforme. Ordene os elementos de $S$ em ordem crescente de valor da função objetivo

Determine um conjunto reduzido $S_{R}$ com os $\gamma k N$ primeiros elementos de $S$

Passo 3. Se $S_{R}=\emptyset$ vá para o Passo 6. Senão selecione $x_{i} \in S_{R}$ e faça $S_{R} \leftarrow S_{R}-\left\{x_{i}\right\}$

Passo 4. Se existe $\tilde{x} \in \tilde{X}$ tal que $\left\|x_{i}-\tilde{x}\right\| \leq r_{k}$ então volte para o Passo 3

Passo 5. $\tilde{x} \leftarrow P\left(x_{i}\right)$

$$
\tilde{X} \leftarrow \tilde{X} \cup\{\tilde{x}\}
$$

Passo 6. $k \leftarrow k+1$

Se $k<K$ volte ao Passo 1

O ponto $y=\operatorname{argmin}\left\{f(\tilde{x}) \mid \tilde{x} \in X^{*}\right\}$ é retornado como aproximação de um minimizador global de $f$.

Este algoritmo, embora teoricamente mais eficaz que o Multistart, apresenta desempenho irregular para problemas com funções objetivo de grau muito maior que 2 ou que não sejam polinomiais. O principal motivo para esta ineficiência deve-se ao fato do algoritmo formar os clusters apenas a partir dos minimizadores locais. Com isto, os clusters formados pelo algoritmo

\footnotetext{
${ }^{2} \mathrm{~A}$ função Gama é definida como [13]:$$
\Gamma(\alpha)=\int_{0}^{\infty} e^{-t} t^{\alpha-1} d t
$$ 
têm sempre o formato de um hipercírculo o que nem sempre é verificado para as regiões de atração dos minimizadores locais de $f$.

Com o intuito de resolver este problema, foi proposto o algoritmo Single Linkage. Este algoritmo foi criado como uma melhoria do Density Clustering. A principal alteração está relacionada a forma em que clusters são criados e atualizados. Para decidir se um dado ponto $x_{k}$ pertence a algum cluster, é verificado se existe algum ponto $x \in S \cup \tilde{X}$ tal que $\left\|x_{k}-x\right\| \leq r_{k}$. Caso exista tal ponto, $x_{k}$ é incluído no mesmo cluster de $x$; do contrário um novo cluster é criado sendo incluído nele $x_{k}$ junto com o minimizador local $P\left(x_{k}\right)$.

Para o cálculo da distância crítica, desejamos reduzir as chances de que $y \in B_{\epsilon}(\Omega)$ não seja encontrado pelo algoritmo. Para isto, queremos que a probabilidade $\alpha$ de que, em uma iteração $k$, $y$ ainda não tenha sido encontrado diminua a cada iteração. Supondo que um minimizador global $x^{*}$ esteja dentro de um cluster hipotético $C$, a probabilidade de que, dados $k N$ pontos iniciais, nenhum deles pertença a $C$ é

$$
\alpha=\left(1-\frac{\mu(C)}{\mu(\Omega)}\right)^{k N},
$$

onde $\mu(C)$ e $\mu(\Omega)$ são as medidas de Lebesgue para o cluster $C$ e $\Omega$, respectivamente.

Visando evitar que o algoritmo termine incorretamente, ou seja que $y$ não tenha sido encontrado, definiremos um valor para $\mu(C)$, e conseqüentemente $r$, de tal forma que $\alpha$ diminua a cada iteração e que o valor de $\mu(C)$ seja o menor possível. Desta forma, para um certo $\sigma>0$, escolhemos $\mu(C)=\frac{\mu(\Omega) \sigma \log (k N)}{k N}$, conforme definido em [24].

Antes de avançarmos na definição de $r$, precisamos definir o cluster $C$ corretamente. Para isto, considere que a região de atração de um minimizador local $\tilde{x}$ é dada por um subconjunto $C$ do conjunto de nível

$$
L(r)=\{x \mid x \in \Omega, f(x) \leq r\},
$$

para algum $r \geq f(\tilde{x})$. Se considerarmos uma aproximação quadrática de $f(x)$ ao redor de $\tilde{x}$ podemos definir $C$ como

$$
C=\left\{x \in \Omega \mid f(\tilde{x})+\frac{1}{2}(x-\tilde{x})^{T} B(x-\tilde{x}) \leq r\right\},
$$

onde $B$ é uma aproximação de $\nabla^{2} f(\tilde{x})$.

Como $C$ é um hipercírculo, então o sua medida de Lebesgue corresponde a $\frac{\pi^{\frac{n}{2}} r^{n}}{\Gamma\left(1+\frac{n}{2}\right)|B|^{\frac{1}{2}}}$.

Temos então que

$$
\mu(C)=\frac{\mu(\Omega) \sigma \log (k N)}{k N}=\frac{\pi^{\frac{n}{2}} r^{n}}{\Gamma\left(1+\frac{n}{2}\right)|B|^{\frac{1}{2}}}
$$

implica

$$
r=\frac{\left[\Gamma\left(1+\frac{n}{2}\right)|B|^{\frac{1}{2}} \mu(\Omega) \sigma \frac{\log (k N)}{k N}\right]^{\frac{1}{n}}}{\sqrt{\pi}} .
$$


Tomando $B=I$ temos

$$
r=\frac{1}{\sqrt{\pi}}\left[\Gamma\left(1+\frac{n}{2}\right) \mu(\Omega) \sigma \frac{\log (k N)}{k N}\right]^{\frac{1}{n}} .
$$

Portanto, o valor da distância crítica utilizada pelo Single Linkage é

$$
r_{k}=\pi^{-\frac{1}{2}}\left(\Gamma\left(1+\frac{n}{2}\right) \mu(\Omega) \sigma \frac{\log k N}{k N} \cdot\right)^{\frac{1}{n}},
$$

onde $\mu(\cdot)$ representa a medida de Lebesgue, $\Gamma(\cdot)$ a função Gama e $\sigma>0$.

O algoritmo descrito a seguir utiliza ainda uma constante $\gamma \in(0,1]$ para criar um subconjunto $S_{R}$ de $S$. Com isto, é possível restringir em $S_{R}$ os pontos que apresentam os menores valores da função objetivo.

\section{Algoritmo 2.4. Single Linkage}

$\operatorname{Dados} K \in \mathbb{N}, N \in \mathbb{N}, X=\emptyset, S=\emptyset$ e $\gamma \in(0,1]$

Passo 0. $k \leftarrow 1$

Passo 1. Calcule $r_{k}$ de acordo com (2.2)

Passo 2. Inclua em $S N$ pontos aleatórios de $\Omega$ com distribuição uniforme. Ordene os elementos de $S$ em ordem crescente de valor da função objetivo

Determine um conjunto reduzido $S_{R}$ com os $\gamma k N$ primeiros elementos de $S$

Passo 3. Se $S_{R}=\emptyset$ então vá para o Passo 6. Senão selecione $x_{i} \in S_{R}$ e faça $S_{R} \leftarrow S_{R}-\left\{x_{i}\right\}$

Passo 4. Se existe $x_{j} \in S_{R} \cup \tilde{X}$ tal que $\left\|x_{i}-x_{j}\right\| \leq r_{k}$ e $f\left(x_{i}\right)>f\left(x_{j}\right)$ então volte para o Passo 3

Passo 5. $\tilde{x} \leftarrow P\left(x_{i}\right)$

$$
\tilde{X} \leftarrow \tilde{X} \cup\{\tilde{x}\}
$$

Passo 6. $k \leftarrow k+1$

Se $k<K$ volte ao Passo 1

O ponto $y=\operatorname{argmin}\left\{f(\tilde{x}) \mid \tilde{x} \in X^{*}\right\}$ é retornado pelo algoritmo como aproximação de um minimizador global de $f$.

A Figura (2.2) mostra um exemplo da aplicação do Density Clustering e do Single Linkage utilizando a função $f(x, y)=\sin (x)+\sin \left(\frac{10 x}{3}\right)+\log (x)-0.84 x+\sin (y)+\sin \left(\frac{10 y}{3}\right)+\log (y)-0.84 y$. Neste exemplo, após a geração de 500 pontos, o Density Clustering identificou 4 clusters distintos, cada qual referente a uma região de atração. Para o mesmo número de pontos, o Single Linkage encontrou 16 clusters diferentes. Os pontos pretos dentro dos círculos coloridos representam os pontos gerados e que foram descartados, ou seja, que não foram utilizados como pontos iniciais pelo procedimento de minimização local. A partir desta figura é possível notar que a quantidade de pontos descartados no Single Linkage é bem maior que a do Density Clustering. 


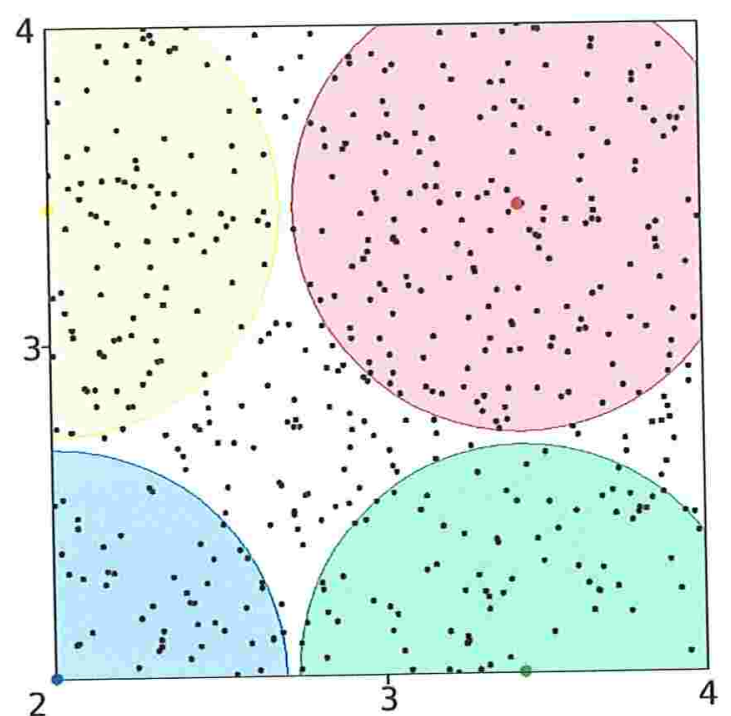

(i) Density Clustering

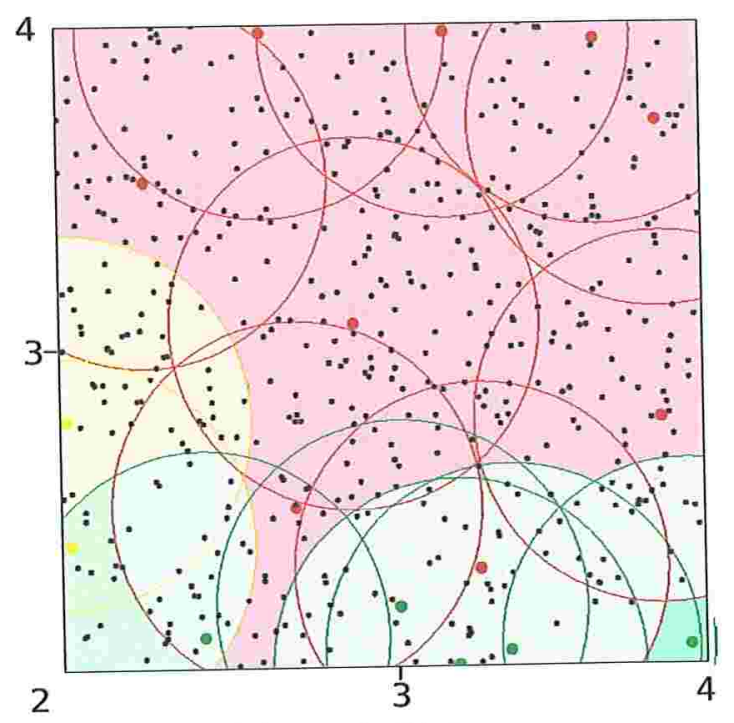

(ii) Single Linkage

Figura 2.2: Comparação entre a abordagem de clusterização utilizada pelo Density Clustering e a utilizada pelo Single Linkage.

\subsection{O algoritmo Random Linkage}

Embora mais eficaz que o Multistart e o Density Clustering, o algoritmo Single Linkage ainda apresenta algumas deficiências, tais como a necessidade de armazenamento dos minimizadores encontrados na Etapa de Refinamento (conjunto $\tilde{X}$ ) e dos pontos aleatórios gerados no início de cada iteração (conjunto $S$ ).

Visando resolver a primeira situação, foram propostas em [19] algumas melhorias para o Single Linkage. Este algoritmo, chamado Random Linkage, substitui o conjunto $S$ por uma função $\varphi$, que calcula a probabilidade da execução do procedimento de busca local a partir de um dado ponto $x_{k+1}$. Esta função é definida como:

$$
\varphi= \begin{cases}1 & \text { se } \delta_{k}>r_{k+1 ; 1 ; \sigma} \\ 0 & \text { caso contrário. }\end{cases}
$$

onde

$$
r_{k+1 ; 1 ; \sigma}=\pi^{-\frac{1}{2}}\left(\Gamma\left(1+\frac{n}{2}\right) \mu(\Omega) \sigma \frac{\log k}{k}\right)^{\frac{1}{n}}
$$

$\mathrm{e}$

$$
\delta_{k}(x)=\min _{j=1, \ldots, k}\left\{\left\|x_{k+1}-x_{j}\right\| \mid f\left(x_{j}\right)<f\left(x_{k+1}\right)\right\} .
$$

Caso não exista $j$ tal que $f\left(x_{j}\right)<f\left(x_{k+1}\right)$ então fazemos $\delta_{k}(x)=\infty$.

Observe que quando $\Omega=\left\{x \in \Re^{n} \mid l \leq x \leq u\right\}, \quad \mu(\Omega)=\prod_{i=1}^{n}\left(u_{i}-l_{i}\right)$ (veja [4]). 


\section{Algoritmo 2.5. Random Linkage}

Dado $K \in \mathbb{N}$

Passo 0. Faça $k \leftarrow 0$

Passo 1. Gere um ponto $x_{k+1}$ de uma distribuição uniforme sobre $\Omega$

Passo 2. Inicie uma busca local em $x_{k+1}$ com probabilidade $\varphi_{k}\left(\delta_{k}\left(x_{k+1}\right)\right)$

Passo 3. $k \leftarrow k+1$

Se $k<K$ volte ao Passo 1

O algoritmo retorna $y$ que corresponde ao ponto encontrado no Passo 2 com menor valor da função objetivo. 


\section{Capítulo 3}

\section{Utilização da curva de Lissajous como estratégia de Tunneling}

\subsection{Introdução}

O método de Tunneling utiliza uma abordagem diferente dos métodos de clusterização na busca por uma boa aproximação de uma solução global. Sua estratégia consiste em minimizar uma função auxiliar $T$ que, dado um minimizador local $\tilde{x}$, nos permita escapar de sua área de atração. Para isto, tomando como ponto inicial $x=\tilde{x}+p$, para $p \in \Re^{n}$ e $\|p\| \ll 1$, e utilizando $T$ como função objetivo, é executado um procedimento de minimização local $P$ em busca de um ponto $x^{u} \in \Omega$ tal que $f\left(x^{u}\right) \leq f(\tilde{x})$ e $x^{u} \neq \tilde{x}$. Para evitar que o procedimento de minimização local seja atraído por $\tilde{x}$, é criado um pólo positivo neste ponto de forma que $\lim _{x \rightarrow \tilde{x}} T(x)=+\infty$. Para a criação deste pólo considere a seguinte função $T$, conforme indicado em $[17,16]$,

$$
T(x)=\frac{f(x)-f(\tilde{x})}{\|x-\tilde{x}\|^{\lambda}} \quad, \lambda \geq 0 .
$$

Supondo $x=\tilde{x}+p$ e, utilizando a aproximação de $2^{a}$ ordem de Taylor, temos

$$
T(x) \cong \frac{p^{t} \nabla f(\tilde{x})+p^{t} \nabla^{2} f(\tilde{x}) p}{\|p\|^{\lambda}} .
$$

Para que se forme o pólo em $\tilde{x}$ queremos que, para pontos muito próximos de $\tilde{x}$, o denominador de $T$ tenda a zero mais rapidamente que seu numerador, ou seja, para $\lim _{\|p\| \rightarrow 0} T(x)$ queremos que

$$
\|p\|^{\lambda}<p^{t} \nabla f(\tilde{x})+p^{t} \nabla^{2} f(\tilde{x}) p .
$$

Aplicando $\ln$ nos dois lados da função temos

$$
\lambda \ln \|p\|<\ln \left[p^{t} \nabla f(\tilde{x})+p^{t} \nabla^{2} f(\tilde{x}) p\right]
$$

que implica

$$
\lambda>\frac{\ln \left[p^{t} \nabla f(\tilde{x})+p^{t} \nabla^{2} f(\tilde{x}) p\right]}{\ln \|p\|} .
$$


Desta forma, para $\lambda$ suficientemente grande, teremos a formação de um pólo positivo em $\tilde{x}$. Na Figura 3.1 observamos um exemplo da função $T$ aplicada a um minimizador local da função $f(x)=\operatorname{sen}(x)+\operatorname{sen}\left(\frac{10 x}{3}\right)+\log (x)-0.84 x$.

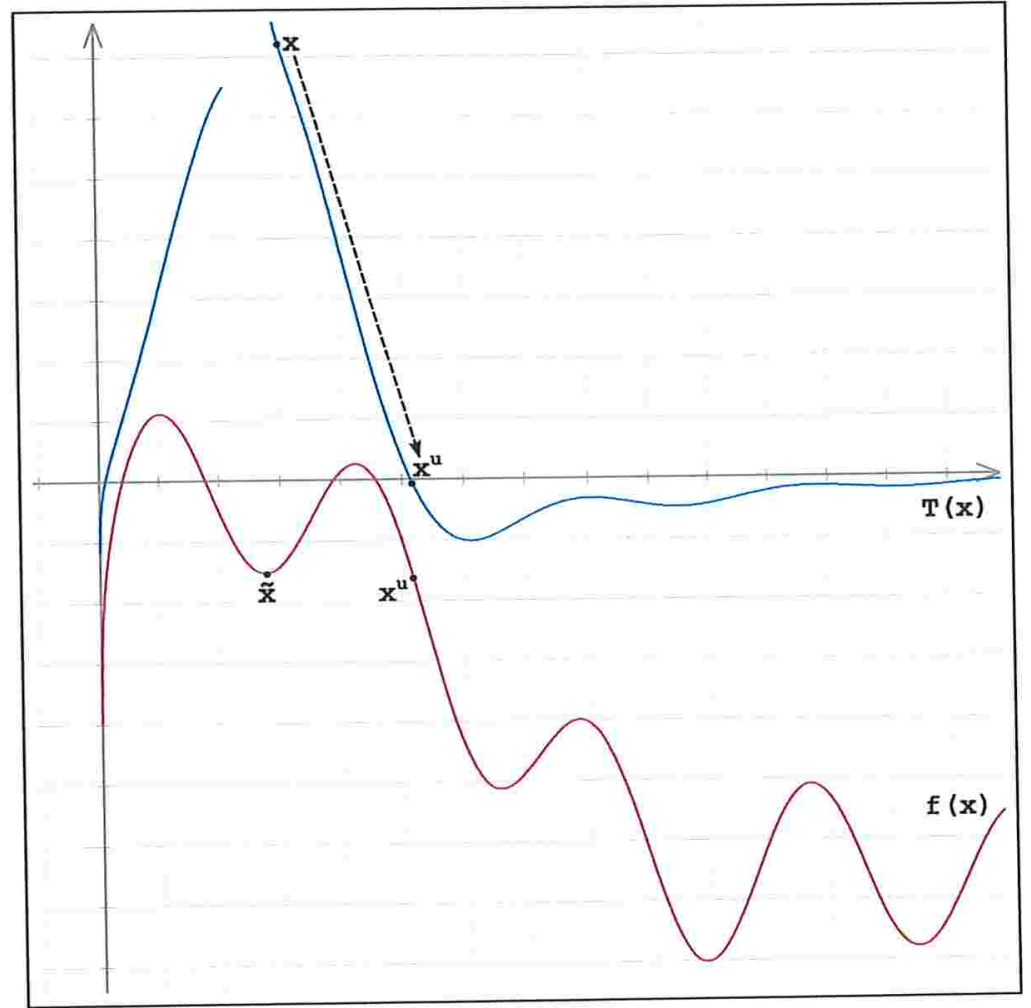

Figura 3.1: Gráfico da função $f(x)=\operatorname{sen}(x)+\operatorname{sen}\left(\frac{10 x}{3}\right)+\log (x)-0.84 x$ e um exemplo da função de tunneling $T(x)$ com um pólo em $\tilde{x}$.

Partindo de um ponto $x$ próximo a $\tilde{x}$, é executado um procedimento de minimização local $P$ onde tenta-se encontrar um ponto $x^{u} \in \Omega$ tal que $T\left(x^{u}\right) \leq 0$. O pólo em $\tilde{x}$ garante que $x^{u} \neq \tilde{x}$. Se $x^{u}$ é encontrado, o processo de minimização da função objetivo é retomado utilizando $x^{u}$ como ponto inicial. Caso contrário, o ponto $\tilde{x}$ é considerado pelo método como sendo um minimizador global da função objetivo. Tomando este exemplo como referência, podemos dividir o método de Tunneling em duas etapas principais:

Fase de Minimização: Sua finalidade é diminuir o valor da função objetivo através da procura de um minimizador local. Dado um ponto inicial $x^{0} \in \Omega$, é utilizado um algoritmo de minimização local $P$ para encontrar um minimizador local $\tilde{x} \in \Omega$.

Fase de Tunneling: Seu propósito é obter um bom ponto inicial para a próxima fase de minimização. Para isto, é utilizada uma função de Tunneling $T(x)$ onde, a partir do minimizador local $\tilde{x}$, é procurado um ponto $x^{u} \in \Omega$ que satisfaça a condição $T\left(x^{u}\right) \leq 0$ para $x^{u} \neq \tilde{x}$. 
Para problemas com vários minimizadores em um mesmo nível, ou seja, um outro minimizador local $x^{u}$ tal que $x^{u} \neq \tilde{x}$ e $f\left(x^{u}\right)=f(\tilde{x})$, pode ocorrer que a saída da fase de Tunneling seja $T\left(x^{u}\right)=0$. Para que o algoritmo não fique alternando entre estes minimizadores locais, é inserido um novo pólo em $x^{u}$ na próxima iteração do algoritmo. Com isto, a função $T(x)$ resultante é definida como:

$$
T(x)=\frac{f(x)-f\left(x^{u}\right)}{\left(\left\|x-x^{u}\right\|^{\lambda_{u}}\right)\left\{\prod_{i=1}^{l-1}\left\|x-\tilde{x}_{i}\right\|^{\lambda_{i}}\right\}},
$$

onde $l$ corresponde ao número de minimizadores locais de mesmo valor encontrados pelo método de Tunneling. Quando a saída da fase de Tunneling não for um minimizador local da função objetivo, $l$ deve voltar ao seu valor inicial $(l=1)$.

É possível que a função de Tunneling seja atraída por um ponto irrelevante ao problema, por exemplo um ponto estacionário $x^{m}$. Para que $T(x)$ saia da região de atração de $x^{m}$, é adicionado um novo termo em $T(x)$ tal que exista um pólo em $x^{m}$ :

$$
T(x)=\frac{f(x)-f\left(\tilde{x}_{l}\right)}{\left\{\prod_{i=1}^{l}\left\|x-x^{i}\right\|^{\lambda_{i}}\right\}\left(\left\|x-x^{m}\right\|^{\lambda_{m}}\right)}
$$

Em alusão ao método descrito até este ponto, o conceito de Tunneling foi generalizado como uma forma de escapar de um minimizador local $\tilde{x}$ através de um novo ponto $x^{u}$ tal que $f\left(x^{u}\right) \leq f(\tilde{x})$. Mais ainda, caso a fase de Tunneling não seja bem sucedida, é possível recomeçar o algoritmo a partir de um novo ponto inicial. O procedimento completo é descrito a seguir:

\section{Algoritmo 3.1. Método de Tunneling}

Dados $J \in \mathbb{N}$ e $K \in \mathbb{N}$

Passo 0. $f_{\min } \leftarrow \infty ; k \leftarrow 1$

Passo 1. Gere um ponto $x_{k} \in \Omega$ com distribuição uniforme

Passo 2. Utilizando $x_{k}$ como ponto inicial, execute $P$ para encontrar um minimizador local $\tilde{x}_{k}$

Passo 3. Utilizando $\tilde{x}_{k}$ como ponto inicial, execute o procedimento de Tunneling na tentativa de encontrar $x_{k}^{u}$ tal que $f\left(x_{k}^{u}\right)<f\left(\tilde{x}_{k}\right)$. Se $x_{k}^{u}$ for encontrado em até $J$ tentativas então faça $x_{k} \leftarrow x_{k}^{u}$ e volte ao Passo 2

Passo 4. Se $f\left(\tilde{x}_{k}\right)<f_{\min }$ então

$$
\begin{aligned}
& f_{\min } \leftarrow f\left(\tilde{x}_{k}\right) \\
& y \leftarrow \tilde{x}_{k}
\end{aligned}
$$

fim se

Passo 5. $k \leftarrow k+1$ 
Se $k<K$ volte ao Passo 1

O ponto $y$ é retornado como aproximação de um minimizador global de $f$.

\section{$3.2 \quad$ A curva de Lissajous}

A curva de Lissajous consiste na trajetória definida para cada solução da função lis : $\Re \longrightarrow \Re^{n}$ dada por

$$
l i s_{i}(t)=a_{i} \cos \left(\theta_{i} t+\varphi_{i}\right), \quad i=1, \ldots, n
$$

onde $a_{i}$ é a amplitude do movimento da curva no componente $i, \theta_{i}$ sua velocidade angular, $\varphi_{i}$ sua posição inicial e $t$ refere-se ao instante atual da curva. Para que a curva de Lissajous não forme ciclos entre seus componentes, é necessário que eles sejam dois a dois independentes, ou seja, a expressão $\theta_{i} / \theta_{j}$ não pode resultar em um número racional. Para evitar esta ocorrência foi criado o conjunto $M$, composto pelos $n$ primeiros números primos tal que $\theta_{i}$ é a raiz quadrada do $i$-ésimo elemento de $M$.

Em [23] foi provado que a curva descrita em (3.5) é suave e densa na região factível $\Omega$ quando as componentes da curva são linearmente independentes. Em particular, foi mostrado que para $t$ tendendo a infinito, a curva de Lisssajous cobrirá todas as regiões de $\Omega$.

Implementamos o Algoritmo 3.1 utilizando o procedimento GENCAN, descrito em [6], para a etapa de minimização local e, no lugar da função (3.3), o procedimento LissajousTunneling na fase de Tunneling. A estratégia utilizada pelo procedimento LissajousTunneling é realizar um movimento por uma curva de Lissajous que passa pelo minimizador local $\tilde{x}$ encontrado pelo GENCAN.

Através da expressão (3.5) é possível construir uma curva densa em $\Omega$ tal que $L(0)=\tilde{x}$. A função $L(\cdot)$ é definida da seguinte forma:

$$
L_{i}(t)=\frac{\left[l_{i}+u_{i}+\left(u_{i}-l_{i}\right) l i s_{i}(t)\right]}{2}, i=1, \ldots, n,
$$

onde $a_{i}=1, i=1, \ldots, n$ e $\varphi_{i}, i=1, \ldots, n$, é escolhido de tal forma que $L(0)=\tilde{x}$.

A Figura 3.2 mostra o gráfico de duas curvas geradas pela função descrita em (3.5).

O movimento ao longo de $L$ é realizado testando-se os candidatos:

$$
t=\frac{\alpha}{(1-|\alpha|)}, \operatorname{com} \alpha=\frac{1}{2},-\frac{1}{2}, \frac{1}{3}, \frac{2}{3},-\frac{1}{3},-\frac{2}{3}, \frac{1}{5}, \frac{2}{5}, \frac{3}{5}, \frac{4}{5}, \ldots
$$

O algoritmo a seguir descreve o procedimento LissajousTunneling:

\section{Algoritmo 3.2. LissajousTunneling}

Dados $\tilde{x}, l, u, n, M$ e $K \in \mathbb{N}$ 


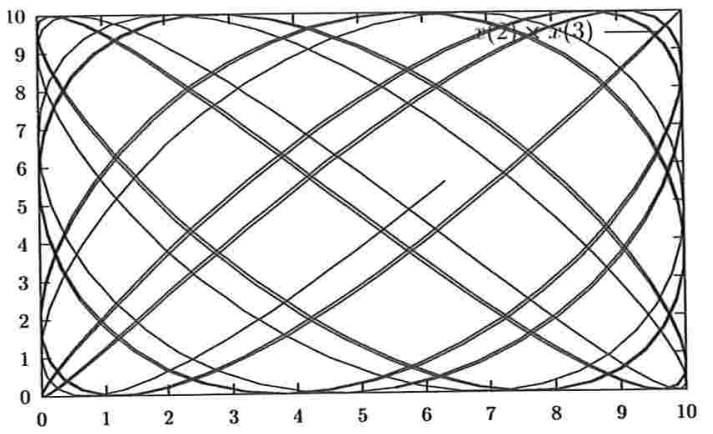

(a)

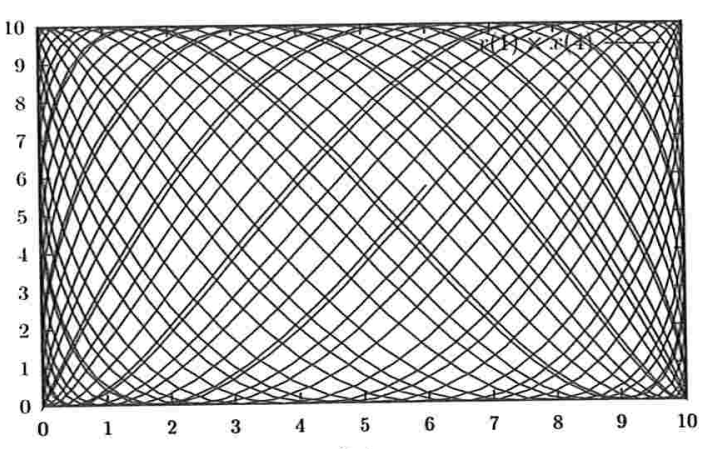

(b)

Figura 3.2: (a) Gráfico de uma curva de Lissajous após percorrer 100 pontos; (b) Gráfico de uma curva de Lissajous após percorrer 1000 pontos.

Passo 0. $x_{\min } \leftarrow \tilde{x} ; k \leftarrow 1$

Passo 1. Para $i \leftarrow 1$ até $n$ faça

$$
\begin{aligned}
& \varphi_{i} \leftarrow \arccos \left(\frac{2 \tilde{x}_{i}-l_{i}-u_{i}}{u_{i}-l_{i}}\right) \\
& \theta_{i} \leftarrow \sqrt{M_{i}}
\end{aligned}
$$

fim para

Passo 2. Calcule o próximo valor de $t$ conforme indicado em (3.6)

Passo 3. $x_{k} \leftarrow \frac{(l+u)+(u-l) \cos (\theta t+\varphi)}{2}$

Passo 4. Se $f\left(x_{\min }\right)>f\left(x_{k}\right)$ então

$$
x_{\min } \leftarrow x_{k}
$$

senão

$$
\begin{aligned}
& k \leftarrow k+1 \\
& \text { se } k<K \text { volte ao Passo } 2
\end{aligned}
$$

fim se

\section{Passo 5. Retorne $x_{\min }$.}

Ao término do Algoritmo 3.2 , se $x_{\min }=\tilde{x}$ então é gerado um novo ponto inicial para a etapa de minimização, do contrário o ponto $x_{\min }$ será utilizado como ponto inicial (Passo 3 do Algoritmo 3.1). 


\subsubsection{Exemplo da utilização da curva de Lissajous}

Para ilustrar uma aplicação prática da curva de Lissajous como estratégia de Tunneling, considere o problema de empacotar 32 círculos de raio 8 em uma caixa de dimensões $100 \times 80$ sem que haja sobreposição entre estes círculos, ou seja, que a maior interseção entre quaisquer dois círculos da caixa seja de no máximo um ponto (para maiores detalhes sobre a formalização deste problema de empacotamento, vide o Capítulo 6). Na Figura (3.3) observamos a execução da $6^{a}$ iteração do algoritmo: em (i) temos a aproximação de um minimizador local encontrado pela Etapa de Refinamento; em (ii), após movimentar o círculo 31, a Fase de Tunneling consegue escapar da região de atração do minimizador local devolvendo um novo ponto para a Etapa de Refinamento; em (iii), com a execução do procedimento de minimização local, o algoritmo devolve uma possível solução para o problema.
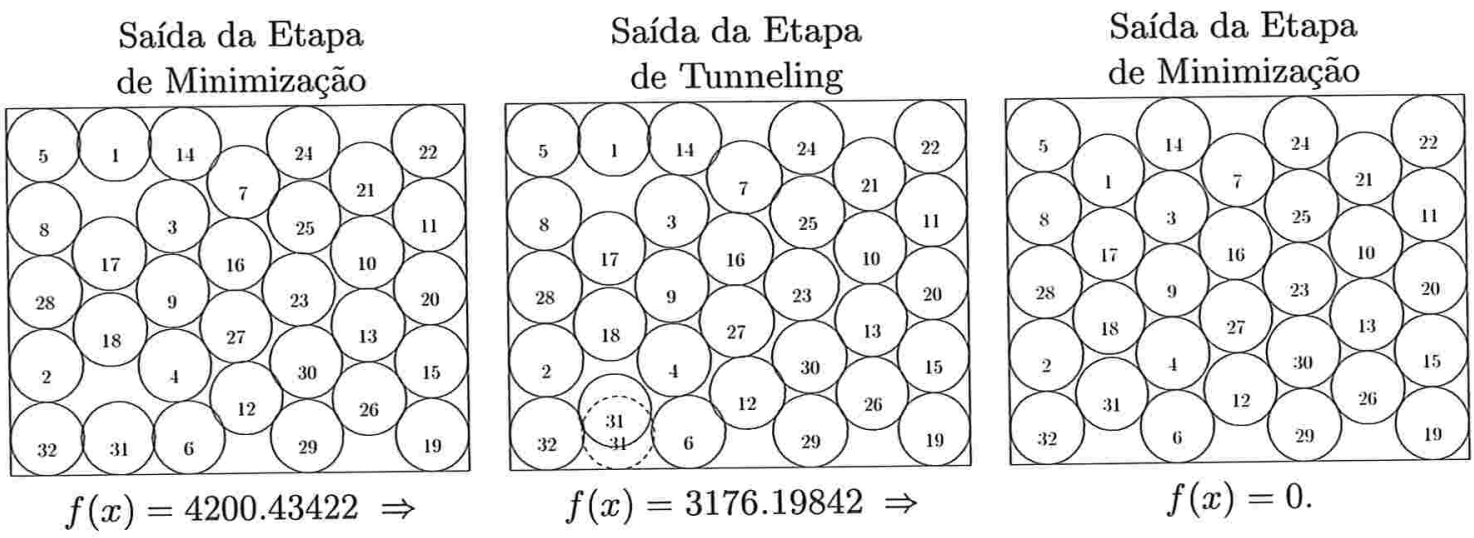

Figura 3.3: Exemplo da execução do algoritmo de Tunneling utilizando a curva de Lissajous para um problema de empacotamento. 


\section{Capítulo 4}

\section{O algoritmo GRASP}

\subsection{Introdução}

Um algoritmo guloso obtém uma solução ótima para um problema de otimização pela resolução de vários subproblemas que, possivelmente, mantêm um grau de dependência entre si. Partindo do problema geral o algoritmo age através de uma sequiência de decisões em que, para cada uma delas, escolhe a opção que julga ser a melhor no momento. A cada escolha o algoritmo avança na resolução dos subproblemas até que o resultado final resolva a questão originalmente proposta.

Dentre os vários algoritmos que utilizam esta técnica destaca-se o GRASP (Greedy Randomized Adaptative Search Procedure), proposto inicialmente em $[11,12]$ para a resolução de problemas de otimização combinatória e, posteriormente, adaptado em $[20,14,15]$ para problemas de otimização contínua.

O GRASP é um algoritmo iterativo composto por duas fases, uma fase de construção e uma fase de busca local. A fase de construção é responsável por calcular, componente a componente, uma nova solução factível $x$. A cada iteração desta fase é escolhida uma componente $[x]_{i}$ de $x$ a partir de uma lista de soluções candidatas, geradas por um procedimento guloso. A escolha da componente dentro do conjunto de soluções candidatas é realizada de forma aleatória, visando evitar que o algoritmo fique parado em um ponto estacionário. Durante a execução da fase de construção, cada componente é escolhida uma única vez, não sendo mais considerada nas iterações subseqüentes desta fase. A fase de busca local realiza um refinamento da solução $x$ encontrada na fase de construção, pela verificação dos valores da função objetivo em um conjunto finito de pontos da vizinhança de $x$. No final do algoritmo, o GRASP retorna como solução o ponto que obteve o menor valor da função objetivo dentre todos os calculados.

\subsection{Elementos principais do algoritmo}

O GRASP surgiu a partir do algoritmo Probalistic heuristic, proposto em [11] como uma variação estocástica da abordagem de Chvátal [8] para o problema de cobertura de conjuntos (set 
covering problem). Posteriormente, o GRASP foi adaptado em [12] para a resolução de problemas gerais de otimização combinatória. No Algoritmo 4.1 é possível observar a estrutura geral do GRASP para a resolução de problemas de combinatória. No Passo 2 é realizada a fase de construção, através da execução do procedimento ConstructGreedyRandomizedSolution. No Passo 3 é realizada a fase de busca local, onde a solução encontrada no passo anterior é refinada em busca de uma melhor aproximação do minimizador global do problema. Por último, é verificado no Passo 5 se o número máximo de iterações foi alcançado.

\section{Algoritmo 4.1. GRASP}

Dados $K \in \mathbb{N} ; \alpha \in(0,1]$

Passo 1. $f^{*} \leftarrow+\infty$

$$
k \leftarrow 1
$$

Passo 2. ConstructGreedyRandomizedSolution $(x, \alpha)$

Passo 3. $\tilde{x} \leftarrow$ LocalSearch $(x)$

Passo 4. Se $f^{*}>f(\tilde{x})$ então

$$
\begin{aligned}
& f^{*} \leftarrow f(\tilde{x}) \\
& x^{*} \leftarrow \tilde{x}
\end{aligned}
$$

fim se

Passo 5. $k \leftarrow k+1$

Se $k<K$ volte ao Passo 2

O ponto $x^{*}$ é retornado pelo algoritmo como aproximação da solução do problema.

No Algoritmo 4.2 explicaremos a Etapa de Construção do GRASP aplicado ao problema do caixeiro viajante (travelling salesman problem). Dadas $n$ cidades e $m$ rotas distintas entre pares de cidades o problema consiste em calcular o custo mínimo de passar por todas as cidades retornando ao ponto de partida. Seja $G$ o grafo tal que o seu conjunto de vértices $V(G)$ representa o conjunto de cidades e o seu conjunto de arestas $A(G)$ representa os possíveis caminhos entre pares de cidades. Suponhamos que as arestas tenham um custo associado as distâncias entre as cidades. Logo, dado um vértice $v$ qualquer o problema consiste em encontrar uma rota que comece e acabe em $v$ e passe por todos os vértices do grafo com custo mínimo. Em cada iteração da Etapa de Construção (Passos $3-7$ ) é incluída uma nova aresta na solução Solution. Este processo, é iniciado com a criação de uma lista de candidatos $S$, formada pelos componentes que podem ser adicionadas a solução parcial que está sendo construída (Passo 2). No Passo 3 são calculados, para todos os elementos de $S$, o valor da função objetivo caso o elemento em questão seja incorporado na solução parcial do problema. Em seguida, no Passo 4, é montado 
um conjunto restrito de soluções $R C L^{1}$ com a seleção dos elementos de $S$ que proporcionam os menores resultados da função objetivo. Esta escolha é baseada em um percentual $\alpha$ de tolerância em relação ao melhor resultado encontrado $\left(c_{\min }\right)$. No Passo 5 é escolhido, de forma aleatória, um elemento $s \in R C L$ para ser incorporado na solução parcial Solution (Passo 6). Este fluxo é repetido até que Solution seja uma solução completa (Passo 7).

\section{Algoritmo 4.2. ConstructGreedyRandomizedSolution}

Dados $x \in \Re^{n} ; \alpha \in(0,1]$

Passo 1. Solution $\leftarrow \emptyset$

Passo 2. Monte uma lista $S$ de componentes candidatos a entrar na solução

Passo 3. Calcule o custo $c(e)$ de incluir cada um destes elementos na solução do problema

Passo 4. $c_{\min } \leftarrow \min \{c(e) \mid e \in S\}$

$$
\begin{aligned}
& c_{\max } \leftarrow \max \{c(e) \mid e \in S\} \\
& R C L \leftarrow\left\{e \in S \mid c(e) \leq c_{\min }+\alpha\left(c_{\max }-c_{\min }\right)\right\}
\end{aligned}
$$

Passo 5. Escolha, aleatoriamente, um elemento $s \in R C L$

Passo 6. Solution $\leftarrow$ Solution $\cup\{s\}$

Passo 7. Se Solution não for uma solução completa volte ao Passo 2

Passo 8. $x \leftarrow$ Solution

Em todo o processo, o valor de $\alpha$ exerce um papel fundamental na qualidade das soluções encontradas. Em problemas de minimização, valores altos de $\alpha$ favorecem o aspecto aleatório do método enquanto valores mais baixos, a escolha gulosa, onde é dada a preferência para os elementos mais próximos de $c_{\text {min }}$. O estudo do comportamento do GRASP em diversos problemas de combinatória favoreceu a compilação de boas estratégias na escolha do conjunto $R C L$. Em [22] são enumeradas algumas, descritas a seguir:

- valor de $\alpha$ fixo, próximo ao caso extremo da escolha gulosa;

- escolha aleatória de $\alpha$ utilizando uma distribuição uniforme;

- escolha aleatória de $\alpha$ utilizando uma distribuição crescente não uniforme;

- escolha periódica de $\alpha$, a partir de um conjunto de valores finitos, de acordo com a qualidade das soluções obtidas (estratégia conhecida como GRASP Reativo).

\footnotetext{
${ }^{1}$ Do original, Restricted Candidates List
} 
Qualquer que seja a estratégia utilizada na escolha do conjunto $R C L$, a solução $x$, gerada pela fase de construção, não é necessariamente ótima, mesma que localmente. Desta forma, a partir da solução encontrada nesta primeira fase, é iniciada uma etapa de refinamento em busca de melhores soluções na vizinhança de $x$. Esta etapa é mostrada no Algoritmo 4.3, que em seu passo único realiza uma busca na vizinhança de $x$, representada por $N(x)$, a procura de um ponto $y$ que apresente menor valor da função objetivo. Em [22] são sugeridas duas formas distintas para a realização da busca em $N(x)$ : a primeira, conhecida como best improving, consiste em avaliar toda a vinhança de $x$ substituindo a solução atual pelo ponto analisado que obteve menor valor da função objetivo; a segunda, conhecida como first improving, consiste em retornar o primeiro ponto que obteve valor da função objetivo menor que a solução atual. Apesar de, na prática, ambas estratégias levarem a mesma solução final, a estratégia first improving foi, freqüentemente, mais eficiente em encontrá-la, conforme descrito em [22].

\section{Algoritmo 4.3. LocalSearch}

Dados $x \in \Re^{n}$

Passo 1. Enquanto $x$ não for localmente ótimo faça

$$
\begin{aligned}
& \text { Encontre } y \in N(x) \text { tal que } f(y)<f(x) \\
& x \leftarrow y
\end{aligned}
$$

fim enquanto

O ponto $x$ resultante é retornado pelo algoritmo como aproximação de um minimizador local do problema.

\subsection{Aplicação do GRASP em problemas de otimização contínua}

Com objetivo de extender a aplicação do GRASP a problemas de otimização contínua foi apresentado em [20] uma nova versão do método para resolução de problemas de programação não linear com restrições em caixa. Uma das características mais marcantes desta versão do GRASP é a não utilização de derivadas. Essa decisão visa dar uma menor complexidade nos cálculos do algoritmo ganhando, com isso, eficiência na resolução de alguns problemas de otimização. Como alternativa ao uso de derivadas para o cálculo de direção e passo que resolvem o problema de PNL são utilizados: uma constante $h>0$, responsável pela determinação do passo do algoritmo, e um conjunto de direções pré-definidas $D$, utilizadas na fase de busca local. O controle do tamanho de $h$ é realizado pela variável NumIterNoImprov de forma tal que, a cada MaxNumIterNoImprov iterações sem melhorar o valor de $f(x)$, o passo é diminuído em $\frac{h}{2}$. O conjunto de direções $D$ define-se como

$$
D(d)=\left\{d \in \Re^{n} \mid d_{i} \in\{-1,0,1\}\right\} .
$$


O número de direções geradas corresponde a $3^{n}-1$, inviabilizando a utilização desta regra para problemas com $n>4$. Nestes casos, a cada iteração do GRASP, é executada apenas a fase de construção. Após $K$ iterações do algoritmo, o ponto $y$ resultante é considerado a aproximação do minimizador global.

Em cada iteração $k$ do GRASP, é definido um novo ponto $x_{k}$. Caso $f\left(x_{k}\right)$ seja menor que a melhor solução encontrada até o momento $\left(f^{*}=f(y)\right.$ ), os valores de $f^{*}$ e $y$ são atualizados com $f\left(x_{k}\right)$ e $x_{k}$, respectivamente. Em linhas gerais, as ações realizadas pelo algoritmo podem ser divididas em três etapas:

- Fase de construção, realizada pelo procedimento guloso ConstructGreedyRandomizedSolution, responsável pelas escolhas que levarão à solução global;

- Fase de busca local, realizado pelo procedimento LocalSearch, aplicado a problemas de até 4 variáveis, responsável por acelerar a identificação da solução. Observamos que, em nossa implementação do GRASP, o procedimento LocalSearch foi substituído pelo GENCAN, conforme será explicado mais detalhadamente no Capítulo 5;

- Conclusão da iteração, onde são atualizados os valores de $f^{*}, y, h$ e NumIterNoImprov.

\section{Algoritmo 4.4. Bloco Principal do GRASP}

Dados $K \in \mathbb{N} ;$ MaxNumIterNoImprov $\in \mathbb{N} ; \alpha \in(0,1]$

Passo 0. $f^{*} \leftarrow+\infty$

$$
\begin{aligned}
& k \leftarrow 1 \\
& h \leftarrow 1 \\
& x_{k} \leftarrow \frac{1}{2}(l+u)
\end{aligned}
$$

Passo 1. Execute o procedimento ConstructGreedyRandomizedSolution $\left(x_{k}, n, h, l, u, \alpha\right)$

Passo 2. Se $n \leq 4$ então

$$
\begin{aligned}
& \text { execute o procedimento LocalSearch }\left(x_{k}, x_{\text {localopt }}, n, l, u, h\right) \\
& x_{k} \leftarrow x_{\text {localopt }}
\end{aligned}
$$

fim se

Passo 4. Se $f^{*}>f\left(x_{k}\right)$ então

$$
\begin{aligned}
& f^{*} \leftarrow f\left(x_{k}\right) \\
& y \leftarrow x_{k} \\
& \text { NumIterNoImprov } \leftarrow 0
\end{aligned}
$$


senão

$$
\text { NumIterNoImprov } \leftarrow \text { NumIterNoImprov }+1
$$

fim se

Passo 5. Se NumIterNoImprov $\geq$ MaxNumIterNoImprov então

$$
\begin{aligned}
& h \leftarrow \frac{h}{2} \\
& \text { NumIterNoImprov } \leftarrow 0
\end{aligned}
$$

fim se

Passo 6. $k \leftarrow k+1$

Se $k<K$ volte ao Passo 1

O ponto $y$ é retornado como aproximação de um minimizador global do problema.

\subsubsection{Fase de construção}

A primeira etapa do GRASP consiste em encontrar um ponto $x_{k}$ que seja uma boa aproximação do minimizador global. Isto é realizado através de sucessivas minimizações, uma para cada componente de $x_{k}$. O vetor $S$ guarda cada um dos índices de $x_{k}$ que ainda não foram minimizados. Para cada índice $i \in S$, são calculados

$$
\begin{aligned}
& {[z]_{i}=\operatorname{argmin}_{t=[l]_{i}+h \beta \leq[u]_{i}, \beta \in \mathbb{N}}\left\{F(t) \equiv f(y) \mid[y]_{i}=t \wedge[y]_{j}=\left[x_{k}\right]_{j} \forall j \neq i\right\},} \\
& {[g]_{i}=F\left([z]_{i}\right)=f\left(\left[x_{k}\right]_{1},\left[x_{k}\right]_{2}, \ldots,[z]_{i}, \ldots,\left[x_{k}\right]_{n}\right),}
\end{aligned}
$$

indicado com a execução do procedimento Minimize no Algoritmo 4.5. As variáveis $g_{\max }$ e $g_{\min }$ guardam, respectivamente, o maior e menor valor encontrados no vetor $g$. Após o cálculo de todos os índices de $S$, aqueles que obtiveram melhor resultado na minimização são colocados em um vetor de candidatos $R C L$ seguindo o seguinte critério: $[g]_{i} \leq g_{\min }+\alpha\left(g_{\max }-g_{\min }\right)$, onde $\alpha$ é uma constante pré-definida. Em seguida é escolhido aleatoriamente um índice $i \in R C L$ sendo $\left[x_{k}\right]_{i}$ atualizado com o valor de $[z]_{i}$. O objetivo desta escolha é evitar que o procedimento gere sempre o mesmo ponto, ficando preso em uma região onde não se encontra o minimizador global. Este índice é então retirado de $S(S=S \backslash\{i\})$. Todo o processo é refeito até que $S$ esteja vazio.

\section{Algoritmo 4.5. ConstructGreedyRandomizedSolution}

Dados $x_{k}, l, u \in \Re^{n}, h \in \Re, \alpha \in(0,1]$

Passo 0. $S \leftarrow\{1,2, \ldots, n\}$

Passo 1. $g_{\min } \leftarrow+\infty$ $g_{\max } \leftarrow-\infty$ 
Para cada elemento $i \in S$ faça

$$
\begin{aligned}
& {[g]_{i} \leftarrow \operatorname{Minimize}\left(x_{k}, h, i, n, l, u,[z]_{i}\right)} \\
& \text { se } g_{\min }>[g]_{i} \text { então } g_{\min } \leftarrow[g]_{i} \\
& \text { se } g_{\max }<[g]_{i} \text { então } g_{\max } \leftarrow[g]_{i}
\end{aligned}
$$

fim para

Passo 2. $R C L \leftarrow \emptyset$

para cada elemento $i \in S$ tal que $[g]_{i} \leq g_{\min }+\alpha\left(g_{\max }-g_{\min }\right)$ faça $R C L \leftarrow R C L \cup\{i\}$

escolha um elemento $j \in R C L$ de forma aleatória

$$
\left[x_{k}\right]_{j} \leftarrow[z]_{j}
$$

Passo 3. $S \leftarrow S \backslash\{j\}$. Enquanto $S$ não estiver vazio, volte ao Passo 1

Devolva o novo $x_{k}$.

\subsubsection{Fase de busca local}

Nesta etapa é realizado um refinamento da solução encontrada na fase de construção. Isto é realizado através da busca por melhores aproximações do minimizador global nas imediações do ponto $x_{k}$. Conforme mencionado em [20], pode ser utilizado para esta tarefa qualquer algoritmo de busca local. Em particular, foi utilizado um algoritmo simples que utiliza-se de direções constantes, indicadas no vetor $D$. Tomando $x_{\text {localopt }}$ como ponto inicial do procedimento, para cada direção $d \in D$ é verificado se $f\left(x_{\text {localopt }}+h d\right)<f\left(x_{\text {localopt }}\right)$. Em caso positivo, faze$\operatorname{mos} x_{\text {localopt }} \leftarrow x_{\text {localopt }}+h d$ e o processo é repetido para todas as direções de $D$. O algoritmo termina quando $\nexists d \in D$ tal que $f\left(x_{\text {localopt }}+h d\right)<f\left(x_{\text {localopt }}\right)$.

\section{Algoritmo 4.7. LocalSearch}

Dados $x_{k}, l, u \in \Re^{n}$ e $h \in \Re$

Passo 0. Gere $D$ conforme descrito na seção (4.1)

$$
\begin{aligned}
& D^{\prime} \leftarrow D \\
& x_{\text {localopt }} \leftarrow x_{k}
\end{aligned}
$$

Passo 1. Selecione uma direção $d \in D$

$$
\begin{aligned}
& D \leftarrow D \backslash\{d\} \\
& \bar{x} \leftarrow x_{\text {localopt }}+h d \\
& \text { se } \bar{x} \text { é factível e } f(\bar{x})<f\left(x_{\text {localopt }}\right) \text { então }
\end{aligned}
$$




$$
\begin{aligned}
& x_{\text {localopt }} \leftarrow \bar{x} \\
& D \leftarrow D^{\prime}
\end{aligned}
$$

fim se

Passo 2. Se $D$ não estiver vazio então vá para o Passo 1

Devolva o novo $x_{\text {localopt }}$.

É válido observar que para problemas de até 4 variáveis, o GRASP assemelha-se a algoritmos que utilizam-se da estratégia de Tunneling, no sentido em que a Etapa de Construção do GRASP tenta melhorar a solução encontrada por uma Etapa de Refinamento anterior.

\subsection{GRASP Contínuo}

Através do estudo do comportamento do GRASP em problemas de PNL foram identificadas algumas deficiências, que contribuiram para uma queda na eficiência do método. Baseado nestas evidências, foram propostas em $[14,15]$ um conjunto de melhorias que resultaram em uma versão alternativa do método denominada GRASP Contínuo (C-GRASP).

O bloco principal do C-GRASP encontra-se descrito no Algoritmo 4.8. Nesta versão, a variável MaxIterNoImprov foi substituída por uma faixa de valores definida pelas constantes $h_{s}$ e $h_{e}$, responsáveis por restringir o tamanho do passo $h$. Esta alteração foi realizada a partir da verificação de que valores muito pequenos de $h$ proporcionavam um aumento considerável no tempo de execução da fase de construção sem que, no entanto, a solução atual fosse melhorada. Além disso, a redução do passo $h$ passou a ser controlada pelos indicadores $\operatorname{Impr}_{c}$ e $\operatorname{Impr}_{l}$ responsáveis por sinalizar mudanças na aproximação do mínimo global $y$. Com isto, $h$ passou a ser reduzido em toda iteração em que não houve alteração de $y$, ao invés de ser alterado a cada MaxIter NoImprov iterações.

\section{Algoritmo 4.8. Bloco Principal do C-GRASP}

Dados $K \in \mathbb{N} ;$ MaxNumIterNoImprov $\in \mathbb{N} ; \alpha \in(0,1]$

Passo 0. $f^{*} \leftarrow+\infty$

$$
k \leftarrow 1
$$

Passo 1. Gere um ponto $x_{k} \in \Omega$ com distribuição uniforme

$$
h \leftarrow h_{s}
$$

Passo 2. $\operatorname{Impr}_{c} \leftarrow F A L S O ; \operatorname{Impr}_{l} \leftarrow F A L S O$

$$
\left[x_{k}, \operatorname{Impr}_{c}\right] \leftarrow \text { ConstructGreedyRandomizedSolution }\left(x_{k}, h, l, u, \alpha\right)
$$


Passo 3. $\left[x_{k}\right.$, Impr $\left._{l}\right] \leftarrow$ LocalSearch $\left(x_{k}, l, u, h, \rho_{l o}\right)$

Passo 4. Se $f^{*}>f\left(x_{k}\right)$ então

$$
\begin{aligned}
& f^{*} \leftarrow f\left(x_{k}\right) \\
& y \leftarrow x_{k}
\end{aligned}
$$

fim se

Passo 5. Se $\operatorname{Impr}_{c}=F A L S O$ e $\operatorname{Impr}_{l}=F A L S O$ então

$$
h \leftarrow \frac{h}{2}
$$

fim se

Passo 6. $k \leftarrow k+1$

Passo 7. Se $k<K$ então

Se $h \geq h_{e}$ volte ao Passo 2

senão volte ao Passo 1

fim se

fim se

O ponto $y$ é retornado como aproximação de um minimizador global de $f$.

A fase de construção encontra-se descrita no Algoritmo 4.9. A principal alteração desta fase foi a criação da variável ReUse com o intuito de sinalizar alterações de valores nas componentes do ponto $x_{k}$. No Passo 1, o procedimento Minimize somente será executado se houver alguma mudança em $x_{k}$. Com isto, evita-se a realização de buscas locais desnecessárias. No Passo 3 é verificado se houve alteração no posicionamento de $x_{k}$. Em caso positivo, ReUse é marcado como $V E R D A D E I R O$ para que $g$ e $z$ sejam atualizados a partir da nova posição de $x_{k}$.

\section{Algoritmo 4.9. ConstructGreedyRandomizedSolution}

Dados $x_{k}, l, u \in \Re^{n}, h \in \Re, \alpha \in(0,1]$

Passo 0. ReUse $\leftarrow$ FALSO

$$
S \leftarrow\{1,2, \ldots, n\}
$$

Passo 1. $g_{\min } \leftarrow+\infty$

$g_{\max } \leftarrow-\infty$

Para cada elemento $i \in S$ faça 
Se $R e U s e=F A L S O$ então

$$
[g]_{i} \leftarrow \operatorname{Minimize}\left(x_{k}, h, i, n, l, u,[z]_{i}\right)
$$

fim se

$$
\begin{aligned}
& \text { se } g_{\min }>[g]_{i} \text { então } g_{\min } \leftarrow[g]_{i} \\
& \text { se } g_{\max }<[g]_{i} \text { então } g_{\max } \leftarrow[g]_{i}
\end{aligned}
$$

fim para

Passo 2. $R C L \leftarrow \emptyset$

para cada elemento $i \in S$ tal que $[g]_{i} \leq g_{\min }+\alpha\left(g_{\max }-g_{\min }\right)$ faça $R C L \leftarrow R C L \cup\{i\}$

Passo 3. Escolha um elemento $j \in R C L$ de forma aleatória

Passo 4. Se $\left[x_{k}\right]_{j}=[z]_{j}$ então

$$
\text { ReUse } \leftarrow \text { VERDADEIRO }
$$

senão

$$
\begin{aligned}
& {\left[x_{k}\right]_{j} \leftarrow[z]_{j}} \\
& \text { ReUse } \leftarrow \text { FALSO } \\
& \text { Impr }_{c} \leftarrow \text { VERDADEIRO }
\end{aligned}
$$

fim se

Passo 5. $S \leftarrow S \backslash\{j\}$. Se $S$ não estiver vazio, volte ao Passo 1

Devolva $x_{k}$ e $I m p r_{c}$.

$\mathrm{Na}$ fase de busca local, a vizinhança de $x_{k}$ é analisada a procura de pontos com menores valores da função objetivo. Se um ponto é encontrado, então $x_{k}$ e $I m p r_{l}$ são atualizados, conforme descrito no Algoritmo 4.10. Para determinação dos pontos da vizinhança de $x_{k}$ que serão analisados, considere

$$
S\left(x_{k}\right)=\left\{\bar{x} \in \Omega \mid \bar{x}=x_{k}+h \tau, \tau \in \mathbb{Z}^{n}\right\},
$$

um subconjunto dos pontos de $\Omega$ que estão a uma quantidade inteira de passos (de tamanho $h$ ) distantes de $x_{k}$. Seja

$$
B_{h}\left(x_{k}\right)=\left\{z \in \Omega \mid z=x_{k}+h \frac{\left(\bar{x}-x_{k}\right)}{\left\|\bar{x}-x_{k}\right\|}, \bar{x} \in S\left(x_{k}\right) \backslash x_{k}\right\}
$$

a projeção dos pontos de $S\left(x_{k}\right) \backslash x_{k}$ em uma hiper-esfera com centro em $x_{k}$ e com raio $h$. 


\section{Algoritmo 4.10. LocalSearch}

Dados $x_{k}, l, u \in \Re^{n}, h \in \Re, \rho_{l o} \in(0,1]$

Passo 0. NumGridPoints $\leftarrow \prod_{i=1}^{n}\left\lceil\frac{\left(u_{i}-l_{i}\right)}{h}\right\rceil$

MaxPointsToExamine $\leftarrow\left\lceil\rho_{l o}\right.$ NumGridPoints $\rceil$

NumPointsExamined $\leftarrow 0$

Passo 1. Escolha aleatóriamente um elemento $z \in B_{h}\left(x_{k}\right)$

Passo 2. Se $z \in[l, u]$ e $f(z)<f\left(x_{k}\right)$ então

$$
\begin{aligned}
& x_{k} \leftarrow z \\
& \text { Impr }_{l} \leftarrow V E R D A D E I R O
\end{aligned}
$$

fim se

Passo 3. NumPointsExamined $\leftarrow$ NumPointsExamined +1

Se NumPointsExamined < MaxPointsToExamine volte ao Passo 1

Devolva $x_{k}$ e Impr .

Em comparação com o Algoritmo 4.7, os pontos analisados não pertencem necessariamente ao grid $x_{k}+h\{-1,0,1\}^{n}$. Espera-se com isto, obter melhores aproximações dos mínimos locais. Além disto, o número de pontos a serem analisados é uma função que utiliza-se do parâmetro definido pelo usuário $\rho_{l o}$ podendo ser adaptada de acordo com o problema a ser resolvido. 


\section{Capítulo 5}

\section{Experimentos computacionais}

\subsection{Introdução}

Para a realização dos experimentos, implementamos em Fortran 77 os métodos Multistart, Random Linkage, GRASP e Tunneling utilizando a curva de Lissajous. Para a Fase de Refinamento (procedimento de minimização local $P$ ) utilizamos o algoritmo GENCAN, proposto em [5]. GENCAN é um algoritmo de restrições ativas utilizado para a resolução de problemas com restrições de caixa que, dentro das faces utiliza o método de Newton truncado com buscas lineares enquanto que, para sair da face, utiliza uma iteração do método do gradiente espectral projetado.

O objetivo deste capítulo é avaliar o desempenho dos métodos mencionados, frente a um conjunto de problemas clássicos de minimização em caixa. Os experimentos foram realizados sobre um conjunto de 52 problemas, propostos em [18, 1], criados a partir de um conjunto de 41 funções (vide Apêndice A para maiores informações sobre as funções utilizadas). Para todos os testes foi considerado como único critério de parada a realização de 2000 iterações, ou seja, 2000 minimizações locais. A execução dos experimentos foi realizada em ambiente Linux de 32 bits (Ubuntu 6.06), em um computador AMD K8 1.8 GHZ com 1 GB de RAM, utilizando o compilador GNU Fortran (GCC) 3.4.6 com o parâmetros de compilação -04.

Para avaliação conjunta dos métodos, convencionamos como iteração a realização de uma Etapa de Refinamento, ou seja, uma execução do GENCAN. Além disso, os algoritmos analisados utilizaram um mesmo conjunto de pontos iniciais de forma a garantir que o $k$-ésimo ponto inicial seria o mesmo para cada um dos algoritmos.

Os resultados dos testes foram divididos em seções de acordo com o método testado. Para cada uma das seções, apresentamos os resultados do método em questão e os comparamos com o método Multistart. Por último, comparamos, de forma sintética, o desempenho dos algoritmos que utilizaram o GENCAN na Etapa de Refinamento. Os resultados foram apresentados em tabelas compostas pelas seguintes colunas:

Prob: problema testado (os problemas encontram-se descritos no Apêndice A);

$n$ : número de variáveis utilizado no teste; 
$f\left(x^{*}\right)$ : valor retornado pelo algoritmo como sendo a aproximação do mínimo global do problema testado;

$i t_{x^{*}}$ : número de iterações realizadas até encontrar $f\left(x^{*}\right)$;

$t_{x^{*}}$ : tempo, em segundos, até encontrar $f\left(x^{*}\right)$;

$f_{\text {eval }}$ : total de avaliações da função objetivo até encontrar o ponto $x^{*}$;

$g_{\text {eval }}$ : total de avaliações do vetor gradiente até encontrar o ponto $x^{*}$;

$\sharp x_{0}$ : total de pontos iniciais gerados até encontrar o ponto $x^{*}$.

A Tabela 5.1 mostra o desempenho do algoritmo Multistart. Como mencionado anteriormente, o método será avaliado nas seções seguintes comparando-o com os outros métodos. 


\begin{tabular}{|c|c|c|c|c|c|c|c|}
\hline \multirow{2}{*}{ Prob } & \multirow[b]{2}{*}{$n$} & \multicolumn{6}{|c|}{ Multistart } \\
\hline & & $f\left(x^{*}\right)$ & $i t_{x^{*}}$ & $t_{x^{*}}$ & $f_{\text {eval }}$ & $g_{\text {eval }}$ & $\sharp x_{0}$ \\
\hline A.1 & 2 & -1.142650 & 1 & 0.00 & $\overline{99}$ & 10 & 1 \\
\hline A. 2 & 2 & -377.608733 & 1 & 0.00 & 8 & 5 & 1 \\
\hline A.3 & 2 & -186.730909 & 31 & 0.01 & 244 & 209 & 31 \\
\hline A.4a & 2 & -186.730909 & 143 & 0.06 & 1486 & 1104 & 143 \\
\hline A.4b & 2 & -186.730909 & 125 & 0.06 & 1348 & 996 & 125 \\
\hline A.5 & 5 & 0.000000 & 27 & 0.02 & 566 & 419 & 27 \\
\hline A.5 & 8 & 0.000000 & 27 & 0.03 & 644 & 471 & 27 \\
\hline A.5 & 10 & 0.000000 & 4 & 0.01 & 148 & 106 & 4 \\
\hline A.5 & 20 & 0.000000 & 50 & 0.30 & 3483 & 2098 & 50 \\
\hline A.5 & 30 & 0.000000 & 6 & 0.16 & 1190 & 701 & 6 \\
\hline A.6 & 4 & -21.392482 & 214 & 0.28 & 7185 & 5438 & 214 \\
\hline A.7 & 2 & -24.062498 & 74 & 0.02 & 529 & 493 & 74 \\
\hline A. 8 & 7 & -274.163160 & 3 & 0.00 & 33 & 29 & 3 \\
\hline A.9 & 2 & -176.541793 & 1 & 0.00 & 8 & 7 & 1 \\
\hline A.10 & 2 & -3.306868 & 1426 & 0.57 & 14797 & 11908 & 1426 \\
\hline A.11 & 3 & -0.490260 & 2 & 0.00 & 21 & 17 & 2 \\
\hline A.12 & 2 & 0.007396 & 678 & 0.23 & 7456 & 4776 & 678 \\
\hline A.13 & 2 & 124.362182 & 1338 & 61.99 & 1024276 & 1025251 & 1338 \\
\hline A.14 & 4 & 0.000307 & 1223 & 24.46 & 503301 & 484418 & 1223 \\
\hline A.15 & 4 & 85822.201600 & 1 & 0.00 & 11 & 12 & 1 \\
\hline A.16 & 5 & 0.000000 & 26 & 0.01 & 285 & 219 & 26 \\
\hline A.17 & 2 & 0.000000 & 1 & 0.00 & 6 & 7 & 1 \\
\hline A.18 & 2 & 0.000000 & 1 & 0.00 & 5 & 6 & 1 \\
\hline A.19a & 4 & -10.153199 & 3 & 0.00 & 40 & 26 & 3 \\
\hline A.19b & 4 & -10.536409 & 3 & 0.00 & 51 & 29 & 3 \\
\hline A.20 & 4 & 0.000000 & 1 & 0.00 & 14 & 15 & 1 \\
\hline A.21 & 2 & -1.031628 & 2 & 0.00 & 19 & 16 & 2 \\
\hline A.22 & 2 & -186.730909 & 31 & 0.01 & 244 & 209 & 31 \\
\hline A.23 & 2 & -78.332331 & 3 & 0.00 & 33 & 31 & 3 \\
\hline A.23 & 3 & -117.498497 & 3 & 0.00 & 35 & 33 & 3 \\
\hline A.23 & 4 & -156.664663 & 40 & 0.02 & 404 & 417 & 40 \\
\hline A.24 & 2 & 0.000000 & 1 & 0.00 & 6 & 6 & 1 \\
\hline A.25 & 2 & -0.407461 & 1 & 0.00 & 13 & 14 & 1 \\
\hline A.26 & 2 & -18.058696 & 1 & 0.00 & 9 & 10 & 1 \\
\hline A.27 & 2 & -227.765750 & 1 & 0.00 & 14 & 15 & 1 \\
\hline A.28 & 2 & -2429.414770 & 1 & 0.00 & 11 & 12 & 1 \\
\hline A.29 & 2 & -2.000000 & 133 & 0.04 & 1272 & 982 & 133 \\
\hline A.30 & 2 & 0.397887 & 1 & 0.00 & 11 & 8 & 1 \\
\hline A.31 & 1 & -3.372897 & 5 & 0.00 & 38 & 30 & 5 \\
\hline A.32 & 2 & 1.000000 & 18 & 0.02 & 310 & 227 & 18 \\
\hline A.33 & 1 & 7.000000 & 1 & 0.00 & 3 & 3 & 1 \\
\hline A.34 & 4 & 0.000000 & 1 & 0.00 & 30 & 21 & 1 \\
\hline A.35 & 2 & 0.000000 & 2 & 0.00 & 24 & 21 & 2 \\
\hline A.36 & 4 & 0.000000 & 1 & 0.00 & 41 & 27 & 1 \\
\hline A.37 & 10 & 0.000000 & 1 & 0.00 & 3 & 4 & 1 \\
\hline A.37 & 20 & 0.000000 & 1 & 0.00 & 7 & 8 & 1 \\
\hline A.37 & 30 & 0.000000 & 1009 & 1.42 & 4778 & 5787 & 1009 \\
\hline A.37 & 40 & 0.000000 & 148 & 0.26 & 651 & 799 & 148 \\
\hline A.38 & 10 & 0.000000 & 1 & 0.00 & 2 & 3 & 1 \\
\hline A.39 & 10 & 2.500000 & 1 & 0.00 & 11 & 12 & 1 \\
\hline A.40 & 10 & 0.000000 & 288 & 0.19 & 5618 & 2893 & 288 \\
\hline A.41 & 1 & -5.534433 & 1 & 0.00 & 7 & 6 & 1 \\
\hline
\end{tabular}

Tabela 5.1: Resultados obtidos a partir da versão implementada do Multistart para um conjunto de problemas propostos em $[18,1]$. 


\subsection{Random Linkage}

Os testes do Random Linkage foram realizados considerando três valores para a constante $\sigma$, utilizada no cálculo da distância crítica $r$. Os valores utilizados, propostos em [18], foram $\sigma \in$ $\{1,2,4\}$. A Tabela 5.2 mostra os resultados.

As três versões do Random Linkage e do Multistart encontraram soluções equivalentes em 48 dos 52 problemas testados. Considerando apenas os 4 problemas restantes, o método Multistart encontrou soluções melhores ou iguais as encontradas por alguma das variantes do Random Linkage em 3 problemas. O Multistart encontrou a melhor solução em 51 dos 52 problemas enquanto que Random Linkage com $\sigma=1,2,4$ encontrou a melhor solução conhecida em 50, 50 e 49 problemas, respectivamente. Nesse sentido, podemos afirmar que o Multistart é levemente mais robusto.

Se consideramos agora os 48 problemas nos quais o Multistart e as três variantes do Random Linkage encontraram soluções de qualidade equivalente, podemos afirmar que as variantes do Random Linkage foram mais eficientes. Por exemplo, o algoritmo Random Linkage com $\sigma=1$ foi mais rápido que o Multistart em 11 problemas, mais lento em 4 problemas e utilizou o mesmo tempo em 33 problemas. A comparação de tempos das outras duas variantes do Random Linkage com o Multistart são similares.

Ao analisar os 11 problemas nos quais o Random Linkake com $\sigma=1$ foi mais rápido do que o Multistart, observamos que em 9 problemas os dois métodos acharam a solução partindo do mesmo ponto inicial. Logo, o ganho em eficiência do Random Linkage é uma consequência de ter descartado pontos iniciais que se encontravam em regiões de atração de minimizadores locais conhecidos. 


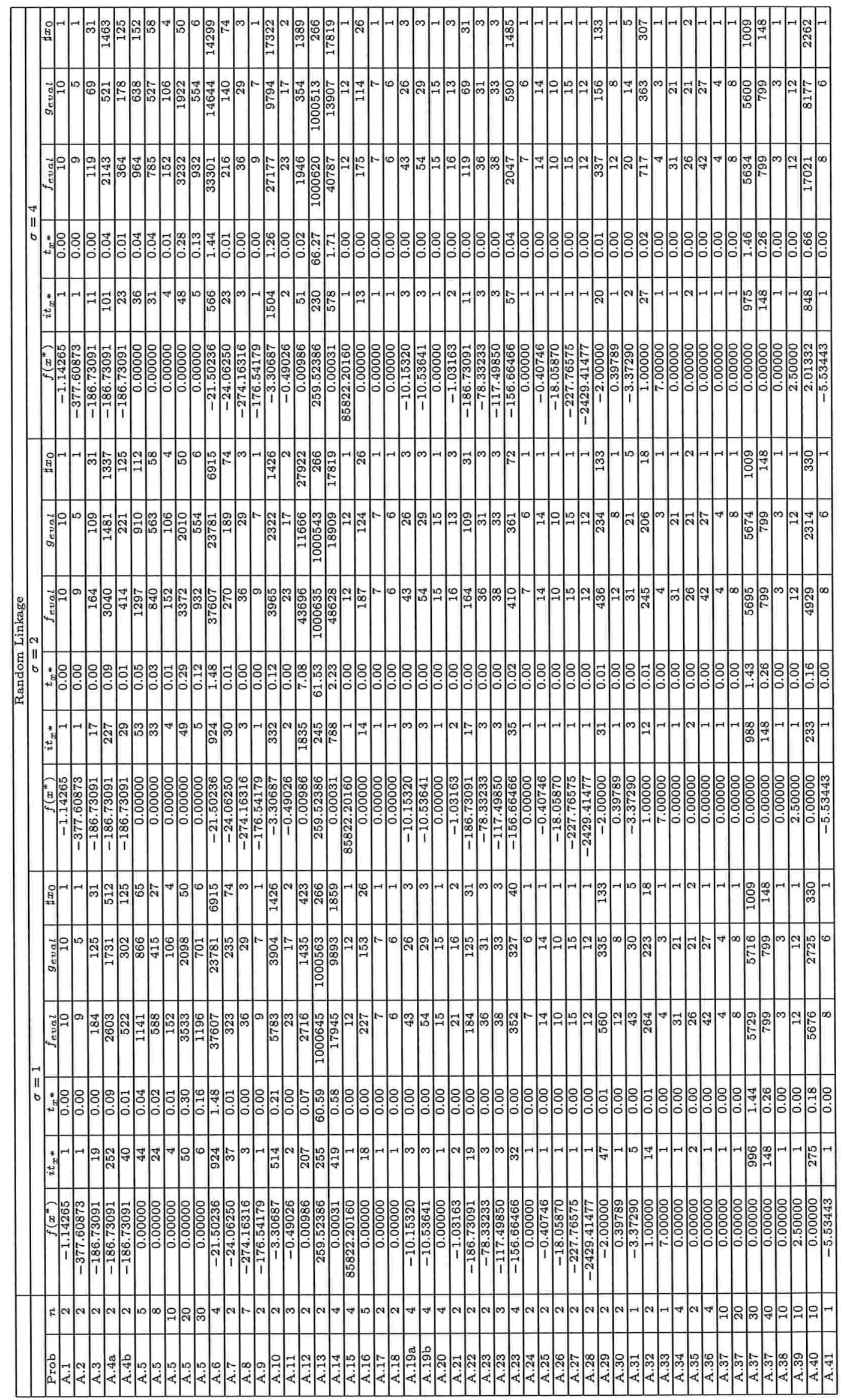

Tabela 5.2: Resultados obtidos a partir das implementações do Random Linkage para um conjunto de problemas propostos em $[18,1]$. 


\subsection{Tunneling}

Os testes com o método de Tunneling foram realizados considerando 2 formas distintas para determinar o valor de $t$ em (3.5), responsável pelo tamanho do passo na curva de Lissajous. A primeira forma, proposta em [23] e que referenciaremos como Lissajous [23], sugere a atualização de $t$ conforme descrito em (3.6). O segundo passo, que referenciaremos como Lissajous(seq), incrementa, a cada iteração do procedimento de Tunneling, o passo em 0.02 . A Tabela 5.3 mostra os resultados.

As duas versões do método de Tunneling e o Multistart encontraram soluções equivalentes em 50 dos 52 problemas testados. Nos 2 problemas restantes, o Lissajous(seq) encontrou soluções melhores que o Multistart. A versão Lissajous [23], por sua vez, saiu-se melhor que o Multistart em apenas um dos citados problemas. Nesse aspecto, podemos considerar o método de Tunneling mais robusto que o Multistart.

Analisando as duas variações testadas do método de Tunneling, o Lissajous(seq) encontrou mais rapidamente a solução esperada em 10 problemas contra 7 do Lissajous [23]. Nos problemas restantes o tempo utilizado pelas duas versões foi o mesmo.

Por último, observamos que o método de Tunneling foi particularmente eficiente para problemas pequenos (com até 5 variáveis) enquanto que, em problemas maiores, seu desempenho foi sensivelmente inferior ao Multistart. Como conseqüência deste fato, para os 50 problemas em que o método de Tunneling obteve soluções equivalentes ao Multistart, ambas as versões do método de Tunneling demoraram, em média, mais tempo para encontrar a solução que o Multistart. 


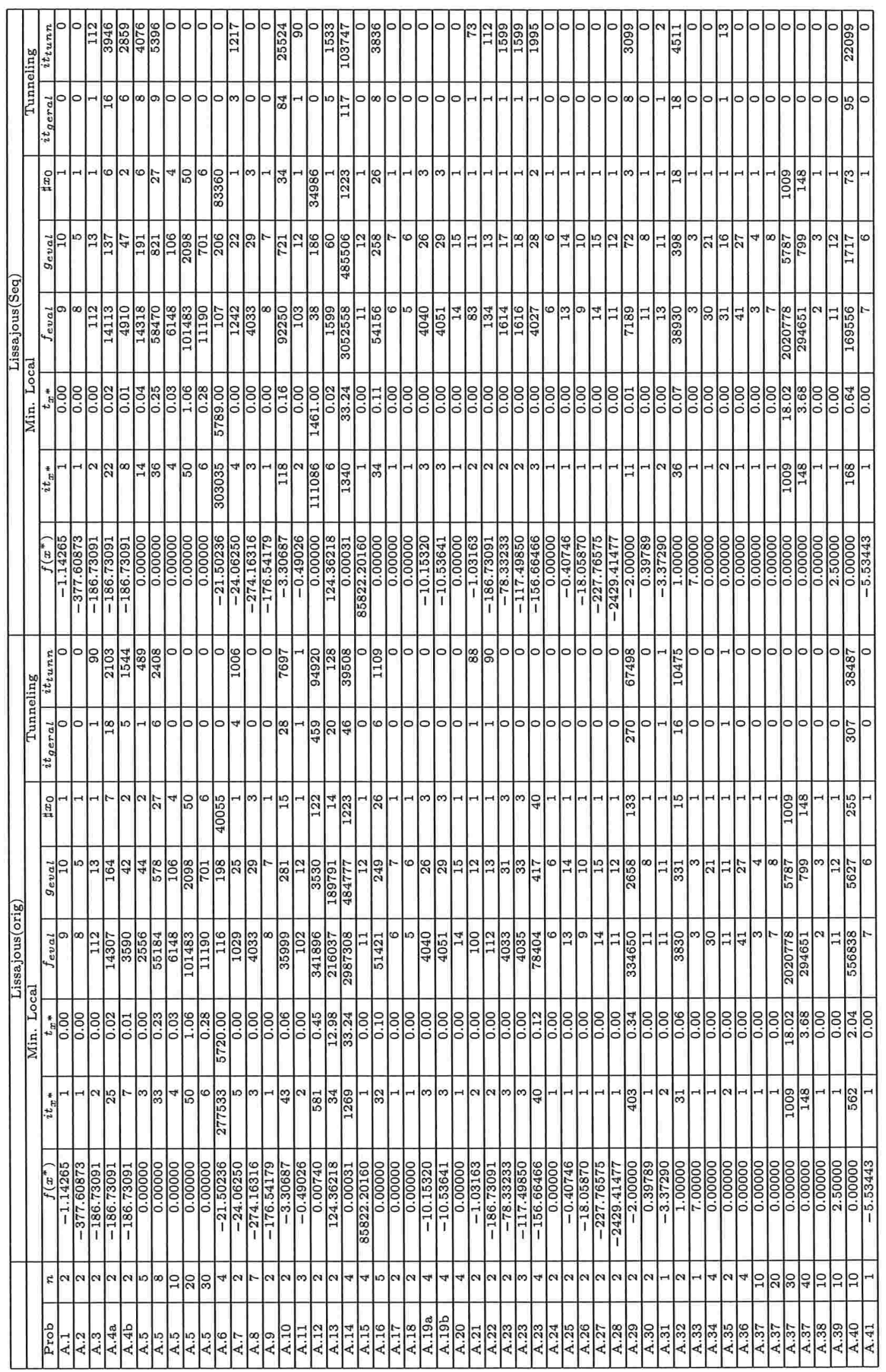

Tabela 5.3: Resultados obtidos a partir das versões implementadas do método de Tunneling, utilizando a curva de Lissajous, para um conjunto de problemas propostos em $[18,1]$. 


\subsection{GRASP}

Para a avaliação do GRASP foram utilizadas três versões distintas do método. A primeira, que referenciaremos apenas como GRASP, consistiu na implementação do algoritmo tal como descrito em [20]. Para a realização dos testes, definimos que o passo $h$, utilizado tanto na Etapa de Construção como de Refinamento do método fosse diminuido em sua metade a cada 20 iterações sem melhora na solução global calculada até aquele momento (parâmetro MaxNumIterNoImprov). Além disso, ao contrário dos demais métodos estudados, esta variação do GRASP utilizou como ponto inicial o ponto $\left(\left(l_{1}+u_{1}\right) / 2, \ldots,\left(l_{n}+u_{n}\right) / 2\right)$.

Na segunda, substituímos a Etapa Refinamento do GRASP pelo GENCAN. Esta versão, que referenciaremos como GRASP-GENCAN, utilizou, ao contrário do GRASP, pontos iniciais aleatórios. Além disso, foi criado o parâmetro MaxNoFunctionImprov, responsável por definir o número máximo de iterações, sem melhora no valor da solução global, antes que um novo ponto inicial seja adotado. Para realização dos testes, utilizamos como valores de MaxNumIterNoImprov, MaxNoFunctionImprov e $\alpha$ as constantes 2, 10 e 0.3, respectivamente.

A última versão, referenciada como C-GRASP-GENCAN, consistiu na implementação do algoritmo descrito em $[14,15]$, onde foram propostas melhorias no GRASP para a resolução de problemas de otimização contínua. Na versão implementada, utilizamos o GENCAN na Etapa de Refinamento do algoritmo e, assim como no GRASP-GENCAN, definimos o parâmetro $\alpha$, utilizado na Etapa de Construção, com o valor de 0.3.

Na Tabela 5.4 verificamos que o GRASP, sem a utilização do GENCAN na Etapa de Refinamento, encontrou soluções melhores que o Multistart em 4 problemas, contra 28 do Multistart. Se considerarmos o tempo que o algoritmo levou para encontrar sua melhor solução o GRASP foi mais rápido em 12 situações contra 23 do Multistart.

Analisando apenas as versões do GRASP que utilizam o GENCAN na Etapa de Refinamento temos que, para os 52 testes realizados, as duas variações do GRASP encontraram a mesma solução que o Multistart em 49 problemas. Nos 3 problemas restantes, ambas as variações do GRASP encontraram uma solução melhor que o Multistart. Neste sentido, podemos afirmar que o GRASP é mais robusto que o Multistart.

Se considerarmos os 49 problemas em que as variações do GRASP utilizando o GENCAN e o Multistart encontraram soluções equivalentes, o GRASP-GENCAN foi mais rápido em 17 problemas, contra 5 do Multistart; enquanto que o C-GRASP-GENCAN, foi mais rápido em 18 problemas contra 3 do Multistart.

Comparando as duas versões do GRASP que utilizam o GENCAN verificamos que o CGRASP-GENCAN foi mais eficiente que o GRASP-GENCAN em 12 problemas. Tal fato é explicado pelo sucesso do C-GRASP em identificar buscas lineares desnecessárias na Etapa de Construção e, com isto, reduzir o número de cálculos de funções. Isto mostra que as melhorias propostas para o C-GRASP-GENCAN aumentaram a eficiência do método. 


\begin{tabular}{|c|c|c|c|c|c|c|c|c|c|c|c|c|}
\hline \multirow[b]{2}{*}{ Prob } & \multirow[b]{2}{*}{$n$} & \multicolumn{6}{|c|}{ Multistart } & \multicolumn{5}{|c|}{ GRASP } \\
\hline & & $f\left(x^{*}\right)$ & $i t_{x^{*}}$ & $t_{x^{*}}$ & $f_{\text {eval }}$ & $g_{\text {eval }}$ & $\forall x_{0}$ & $f\left(x^{*}\right)$ & $i t_{x^{*}}$ & $t_{x *}$ & $f_{\text {eval }}$ & $\forall x_{0}$ \\
\hline A. 1 & 2 & -1.142650 & 1 & 0.00 & 9 & 10 & 1 & -1.142647 & 84 & 0.01 & 20796 & 1 \\
\hline A. 2 & 2 & -377.608733 & 1 & 0.00 & 8 & 5 & 1 & -377.584375 & 43 & 0.00 & 1578 & 1 \\
\hline A.3 & 2 & -186.730909 & 31 & 0.01 & 244 & 209 & 31 & -186.721815 & 129 & 0.07 & 86046 & 1 \\
\hline A.4a & 2 & -186.730909 & 143 & 0.06 & 1486 & 1104 & 143 & -186.338941 & 168 & 0.35 & 329474 & 1 \\
\hline A.4b & 2 & -186.730909 & 125 & 0.06 & 1348 & 996 & 125 & -186.729447 & 431 & 10.46 & 10182526 & 2 \\
\hline A. 5 & 5 & 0.000000 & 27 & 0.02 & 566 & 419 & 27 & 0.000000 & 3 & 0.00 & 948 & 1 \\
\hline A.5 & 8 & 0.000000 & 27 & 0.03 & 644 & 471 & 27 & 0.000000 & 3 & 0.00 & 2271 & 1 \\
\hline A.5 & 10 & 0.000000 & 4 & 0.01 & 148 & 106 & 4 & 0.000000 & 3 & 0.01 & 3468 & 1 \\
\hline A.5 & 20 & 0.000000 & 50 & 0.30 & 3483 & 2098 & 50 & 0.000000 & 5 & 0.14 & 22055 & 1 \\
\hline A.5 & 30 & 0.000000 & 6 & 0.16 & 1190 & 701 & 6 & 0.000000 & 5 & 0.45 & 48830 & 1 \\
\hline A.6 & 4 & -21.392482 & 214 & 0.28 & 7185 & 5438 & 214 & -21.500000 & 42 & 0.03 & 16956 & 1 \\
\hline A.7 & 2 & -24.062498 & 74 & 0.02 & 529 & 493 & 74 & -24.062302 & 148 & 0.15 & 173972 & 1 \\
\hline A. 8 & 7 & -274.16316 & 3 & 0.00 & 33 & 29 & 3 & -274.14845 & 109 & 0.25 & 148789 & 1 \\
\hline A.9 & 2 & -176.541793 & 1 & 0.00 & 8 & 7 & 1 & -176.525861 & 129 & 0.07 & 86049 & 1 \\
\hline A.10 & 2 & -3.306868 & 1426 & 0.57 & 14797 & 11908 & 1426 & -3.208125 & 233 & 0.24 & 270618 & 1 \\
\hline A.11 & 3 & -0.490260 & 2 & 0.00 & 21 & 17 & 2 & -0.490256 & 469 & 0.24 & 114652 & 3 \\
\hline A.12 & 2 & 0.007396 & 678 & 0.23 & 7456 & 4776 & 678 & 0.000000 & 1 & 0.00 & 3614 & 1 \\
\hline A.13 & 2 & 124.362182 & 1338 & 61.99 & 1024276 & 1025251 & 1338 & 124.362204 & 146 & 33.47 & 8353672 & 1 \\
\hline A.14 & 4 & 0.000307 & 1223 & 24.46 & 503301 & 484418 & 1223 & 0.001035 & 188 & 3399.86 & 1955334997 & 1 \\
\hline A.15 & 4 & 85822.2016 & 1 & 0.00 & 11 & 12 & 1 & 85826.7279 & 84 & 8.34 & 753037 & 1 \\
\hline A.16 & 5 & 0.000000 & 26 & 0.01 & 285 & 219 & 26 & 0.000000 & 1 & 0.00 & 316 & 1 \\
\hline A.17 & 2 & 0.000000 & 1 & 0.00 & 6 & 7 & 1 & 0.000000 & 2 & 0.00 & 110 & 1 \\
\hline A.18 & 2 & 0.000000 & 1 & 0.00 & 5 & 6 & 1 & 0.000000 & 1 & 0.00 & 29 & 1 \\
\hline A.19a & 4 & -10.153199 & 3 & 0.00 & 40 & 26 & 3 & -10.153195 & 1 & 0.00 & 194 & 1 \\
\hline A.19b & 4 & -10.536409 & 3 & 0.00 & 51 & 29 & 3 & $-10,536283$ & 1 & 0.00 & 280 & 1 \\
\hline A.20 & 4 & 0.000000 & 1 & 0.00 & 14 & 15 & 1 & 0.000000 & 1 & 0.00 & 153 & 1 \\
\hline A.21 & 2 & -1.031628 & 2 & 0.00 & 19 & 16 & 2 & -1.031551 & 147 & 0.02 & 34830 & 1 \\
\hline A.22 & 2 & -186.730909 & 31 & 0.01 & 244 & 209 & 31 & -186.721815 & 129 & 0.07 & 86046 & 1 \\
\hline A.23 & 2 & -78.332331 & 3 & 0.00 & 33 & 31 & 3 & -78.332076 & 103 & 0.04 & 80770 & 1 \\
\hline A.23 & 3 & -117.498497 & 3 & 0.00 & 35 & 33 & 3 & -117.498114 & 103 & 0.12 & 162319 & 1 \\
\hline A.23 & 4 & -156.664663 & 40 & 0.02 & 404 & 417 & 40 & -156.664152 & 103 & 0.28 & 274359 & 1 \\
\hline A.24 & 2 & 0.000000 & 1 & 0.00 & 6 & 6 & 1 & 0.000000 & 1 & 0.00 & 44 & 1 \\
\hline A.25 & 2 & -0.407461 & 1 & 0.00 & 13 & 14 & 1 & -0.407461 & 166 & 0.14 & 165374 & 1 \\
\hline A.26 & 2 & -18.058696 & 1 & 0.00 & 9 & 10 & 1 & -18.058686 & 125 & 0.03 & 42760 & 1 \\
\hline A.27 & 2 & -227.76575 & 1 & 0.00 & 14 & 15 & 1 & -227.765422 & 125 & 0.17 & 163390 & 1 \\
\hline A.28 & 2 & -2429.41477 & 1 & 0.00 & 11 & 12 & 1 & -2429.21468 & 63 & 0.02 & 19002 & 1 \\
\hline A.29 & 2 & -2.000000 & 133 & 0.04 & 1272 & 982 & 133 & -2.000000 & 1 & 0.00 & 44 & 1 \\
\hline A.30 & 2 & 0.397887 & 1 & 0.00 & 11 & 8 & 1 & 0.397922 & 128 & 0.09 & 168870 & 1 \\
\hline A.31 & 1 & -3.372897 & 5 & 0.00 & 38 & 30 & 5 & -3.372883 & 82 & 0.00 & 13172 & 1 \\
\hline A.32 & 2 & 1.000000 & 18 & 0.02 & 310 & 227 & 18 & 1.000000 & 32 & 0.00 & 32 & 1 \\
\hline A.33 & 1 & 7.000000 & 1 & 0.00 & 3 & 3 & 1 & 7.000000 & 1 & 0.00 & 12 & 1 \\
\hline A.34 & 4 & 0.000000 & 1 & 0.00 & 30 & 21 & 1 & 0.000000 & 1 & 0.00 & 316 & 1 \\
\hline A.35 & 2 & 0.000000 & 2 & 0.00 & 24 & 21 & 2 & 0.000000 & 22 & 0.00 & 668 & 1 \\
\hline A.36 & 4 & 0.000000 & 1 & 0.00 & 41 & 27 & 1 & 0.000000 & 1 & 0.00 & 133 & 1 \\
\hline A.37 & 10 & 0.000000 & 1 & 0.00 & 3 & 4 & 1 & 0.000000 & 4 & 0.00 & 6164 & 1 \\
\hline A.37 & 20 & 0.000000 & 1 & 0.00 & 7 & 8 & 1 & 0.000000 & 6 & 0.14 & 35286 & 1 \\
\hline A.37 & 30 & 0.000000 & 1009 & 1.42 & 4778 & 5787 & 1009 & 0.000000 & 5 & 0.47 & 65105 & 1 \\
\hline A.37 & 40 & 0.000000 & 148 & 0.26 & 651 & 799 & 148 & 0.000000 & 3 & 0.69 & 68883 & 1 \\
\hline A.38 & 10 & 0.000000 & 1 & 0.00 & 2 & 3 & 1 & 0.000000 & 3 & 0.00 & 2973 & 1 \\
\hline A.39 & 10 & 2.500000 & 1 & 0.00 & 11 & 12 & 1 & 2.500000 & 3 & 0.00 & 2973 & 1 \\
\hline A.40 & 10 & 0.000000 & 288 & 0.19 & 5618 & 2893 & 288 & 0.000000 & 3 & 0.00 & 5118 & 1 \\
\hline A.41 & 1 & -5.534433 & 1 & 0.00 & 7 & 6 & 1 & -5.534432 & 123 & 0.00 & 8527 & 1 \\
\hline
\end{tabular}

Tabela 5.4: Resultados obtidos a partir da versão implementada do GRASP para um conjunto de problemas propostos em $[18,1]$. 


\begin{tabular}{|c|c|c|c|c|c|c|c|c|c|c|c|c|c|}
\hline \multirow[b]{2}{*}{ Prob } & \multirow[b]{2}{*}{$n$} & \multicolumn{6}{|c|}{ GRASP-GENCAN } & \multicolumn{6}{|c|}{ C-GRASP-GENCAN } \\
\hline & & $f\left(x^{*}\right)$ & $i t_{x} *$ & $t_{x *}$ & $f_{\text {eval }}$ & $g_{\text {eval }}$ & $\sharp x_{0}$ & $f\left(x^{*}\right)$ & $i t_{x^{*}}$ & $t_{x^{*}}$ & $f_{\text {eval }}$ & $g_{\text {eval }}$ & $\forall x_{0}$ \\
\hline A.1 & 2 & -1.142650 & 1 & 0.00 & 69 & 7 & 1 & -1.142650 & 1 & 0.00 & 72 & 7 & 1 \\
\hline A.2 & 2 & -377.608733 & 1 & 0.00 & 23 & 7 & 1 & -377.608733 & 1 & 0.00 & 26 & 7 & 1 \\
\hline A.3 & 2 & -186.730909 & 1 & 0.00 & 69 & 6 & 1 & -186.730909 & 1 & 0.00 & 72 & 6 & 1 \\
\hline A.4a & 2 & -186.730909 & 22 & 0.02 & 16417 & 107 & 2 & -186.730909 & 10 & 0.00 & 1324 & 38 & 2 \\
\hline A.4b & 2 & -186.730909 & 8 & 0.00 & 907 & 48 & 1 & -186.730909 & 6 & 0.00 & 655 & 26 & 1 \\
\hline A.5 & 5 & 0.000000 & 1 & 0.00 & 356 & 27 & 1 & 0.000000 & 1 & 0.00 & 305 & 27 & 1 \\
\hline A.5 & 8 & 0.000000 & 1 & 0.00 & 780 & 20 & 1 & 0.000000 & 1 & 0.00 & 596 & 27 & 1 \\
\hline A.5 & 10 & 0.000000 & 1 & 0.00 & 1183 & 21 & 1 & 0.000000 & 1 & 0.00 & 666 & 21 & 1 \\
\hline A.5 & 20 & 0.000000 & 1 & 0.04 & 4479 & 45 & 1 & 0.000000 & 1 & 0.02 & 2885 & 45 & 1 \\
\hline A.5 & 30 & 0.000000 & 1 & 0.10 & 9835 & 50 & 1 & 0.000000 & 1 & 0.07 & 6846 & 50 & 1 \\
\hline A.6 & 4 & -21.502356 & 7 & 0.00 & 2483 & 20 & 1 & -21.502356 & 5 & 0.00 & 1193 & 16 & 1 \\
\hline A.7 & 2 & -24.062498 & 6 & 0.00 & 456 & 23 & 1 & -24.062498 & 5 & 0.00 & 381 & 17 & 1 \\
\hline A.8 & 7 & -274.163160 & 1 & 0.00 & 238 & 11 & 1 & -274.163160 & 1 & 0.00 & 211 & 11 & 1 \\
\hline A.9 & 2 & -176.541793 & 1 & 0.00 & 68 & 6 & 1 & -176.541793 & 1 & 0.00 & 71 & 6 & 1 \\
\hline A.10 & 2 & -3.306868 & 108 & 0.06 & 28984 & 559 & 10 & -3.306868 & 464 & 0.11 & 11092 & 1624 & 74 \\
\hline A.11 & 3 & -0.490260 & 32 & 0.02 & 1501 & 210 & 4 & -0.490260 & 6 & 0.00 & 116 & 25 & 2 \\
\hline A.12 & 2 & 0.000000 & 12 & 0.10 & 192643 & 40 & 2 & 0.000000 & 11 & 0.03 & 63057 & 33 & 2 \\
\hline A.13 & 2 & 124.362182 & 1 & 0.01 & 3014 & 8 & 1 & 124.362182 & 1 & 0.01 & 3017 & 8 & 1 \\
\hline A.14 & 4 & 0.001036 & 64 & 64.47 & 38994713 & 4115 & 4 & 0.000941 & 338 & 10.28 & 6293840 & 1552 & 39 \\
\hline A.15 & 4 & 85822.201600 & 1 & 0.02 & 2019 & 10 & 1 & 85822.201600 & 1 & 0.02 & 1827 & 10 & 1 \\
\hline A.16 & 5 & 0.000000 & 12 & 0.02 & 19402 & 32 & 2 & 0.000000 & 27 & 0.01 & 9686 & 72 & 6 \\
\hline A.17 & 2 & 0.000000 & 1 & 0.00 & 43 & 5 & 1 & 0.000000 & 1 & 0.00 & 46 & 5 & 1 \\
\hline A.18 & 2 & 0.000000 & 1 & 0.00 & 19 & 5 & 1 & 0.000000 & 1 & 0.00 & 22 & 5 & 1 \\
\hline A.19a & 4 & -10.153199 & 1 & 0.00 & 134 & 10 & 1 & -10.153199 & 1 & 0.00 & 132 & 10 & 1 \\
\hline A.19b & 4 & -10.536409 & 1 & 0.00 & 128 & 8 & 1 & -10.536409 & 1 & 0.00 & 126 & 8 & 1 \\
\hline A.20 & 4 & 0.000000 & 1 & 0.00 & 83 & 14 & 1 & 0.000000 & 1 & 0.00 & 85 & 14 & 1 \\
\hline A.21 & 2 & -1.031628 & 1 & 0.00 & 20 & 6 & 1 & -1.031628 & 1 & 0.00 & 23 & 6 & 1 \\
\hline A.22 & 2 & -186.730909 & 1 & 0.00 & 69 & 6 & 1 & -186.730909 & 1 & 0.00 & 72 & 6 & 1 \\
\hline A.23 & 2 & -78.332331 & 2 & 0.00 & 258 & 14 & 1 & -78.332331 & 2 & 0.00 & 264 & 14 & 1 \\
\hline A.23 & 3 & -117.498497 & 1 & 0.00 & 255 & 6 & 1 & -117.498497 & 1 & 0.00 & 261 & 6 & 1 \\
\hline A.23 & 4 & -156.664663 & 2 & 0.00 & 833 & 12 & 1 & -156.664663 & 2 & 0.00 & 685 & 12 & 1 \\
\hline A.24 & 2 & 0.000000 & 1 & 0.00 & 35 & 3 & 1 & 0.000000 & 1 & 0.00 & 38 & 3 & 1 \\
\hline A.25 & 2 & -0.407461 & 1 & 0.00 & 42 & 3 & 1 & -0.407461 & 1 & 0.00 & 45 & 3 & 1 \\
\hline A.26 & 2 & -18.058696 & 1 & 0.00 & 37 & 5 & 1 & -18.058696 & 1 & 0.00 & 40 & 5 & 1 \\
\hline A.27 & 2 & -227.765750 & 1 & 0.00 & 134 & 12 & 1 & -227.765750 & 1 & 0.00 & 137 & 12 & 1 \\
\hline A.28 & 2 & -2429.414770 & 1 & 0.00 & 130 & 8 & 1 & -2429.414770 & 1 & 0.00 & 133 & 8 & 1 \\
\hline A.29 & 2 & -2.000000 & 2 & 0.00 & 74 & 8 & 1 & -2.000000 & 2 & 0.00 & 80 & 8 & 1 \\
\hline A.30 & 2 & 0.397887 & 1 & 0.00 & 126 & 4 & 1 & 0.397887 & 1 & 0.00 & 129 & 4 & 1 \\
\hline A.31 & 1 & -3.372897 & 1 & 0.00 & 44 & 4 & 1 & -3.372897 & 1 & 0.00 & 45 & 4 & 1 \\
\hline A.32 & 2 & 1.000000 & 23 & 0.01 & 2466 & 130 & 3 & 1.000000 & 11 & 0.00 & 482 & 29 & 3 \\
\hline A.33 & 1 & 7.000000 & 1 & 0.00 & 8 & 2 & 1 & 7.000000 & 1 & 0.00 & 9 & 2 & 1 \\
\hline A.34 & 4 & 0.000000 & 1 & 0.00 & 88 & 13 & 1 & 0.000000 & 1 & 0.00 & 90 & 13 & 1 \\
\hline A.35 & 2 & 0.000000 & 1 & 0.00 & 28 & 10 & 1 & 0.000000 & 1 & 0.00 & 31 & 10 & 1 \\
\hline A.36 & 4 & 0.000000 & 1 & 0.00 & 71 & 17 & 1 & 0.000000 & 1 & 0.00 & 88 & 23 & 1 \\
\hline A.37 & 10 & 0.000000 & 1 & 0.00 & 1543 & 4 & 1 & 0.000000 & 1 & 0.00 & 1395 & 4 & 1 \\
\hline A.37 & 20 & 0.000000 & 3 & 0.06 & 17647 & 10 & 1 & 0.000000 & 3 & 0.05 & 13579 & 10 & 1 \\
\hline A.37 & 30 & 0.000000 & 2 & 0.15 & 26045 & 7 & 1 & 0.000000 & 2 & 0.13 & 21407 & 7 & 1 \\
\hline A. 37 & 40 & 0.000000 & 2 & 0.37 & 45925 & 7 & 1 & 0.000000 & 2 & 0.28 & 35356 & 7 & 1 \\
\hline A.38 & 10 & 0.000000 & 1 & 0.00 & 992 & 3 & 1 & 0.000000 & 1 & 0.00 & 781 & 3 & 1 \\
\hline A.39 & 10 & 2.500000 & 1 & 0.00 & 1000 & 11 & 1 & 2.500000 & 1 & 0.00 & 770 & 11 & 1 \\
\hline A.40 & 10 & 0.000000 & 3 & 0.01 & 5147 & 18 & 1 & 0.000000 & 3 & 0.00 & 3136 & 18 & 1 \\
\hline A.41 & 1 & -5.534433 & 1 & 0.00 & 9 & 4 & 1 & -5.534433 & 1 & 0.00 & 10 & 4 & 1 \\
\hline
\end{tabular}

Tabela 5.5: Resultados obtidos a partir das versões implementadas do GRASP, utilizando o GENCAN na Fase de Refinamento, para um conjunto de problemas propostos em $[18,1]$. 


\subsection{Comparativo dos resultados}

De um modo geral, as soluções encontradas pelos métodos foram muito próximas. Dos 52 problemas avaliados, os métodos encontraram soluções equivalentes em 47 problemas.

Para comparação do desempenho dos métodos utilizaremos gráficos de performance (performance profiles), propostos em [10]. Para isto, considere a seguinte formulação: seja o teste de $n$ problemas aplicados a $m$ métodos diferentes. Considere $t_{i, j}$ o tempo que o método $j$ gastou para encontrar uma aproximação do minimizador global para o problema $i$. Visando guardar os melhores resultados para cada problema, considere $\tilde{t}_{i}=\min \left\{t_{i, j} \mid j=1, \ldots, m\right\}$. Suponha $T_{k, j}=$ número de testes em que $\left.t_{i, j} \leq k \tilde{t}_{i} \mid i=1, \ldots, n\right\}$, o gráfico de performance de um método $j$ é representado da seguinte forma $g_{j}=\left\{\left(k, T_{k, j} / n\right) \mid k \geq 1\right\}$. Desta forma, por exemplo, o par ordenado $(5,0.8)$ de um certo método $j$ indica que o método conseguiu encontrar uma boa aproximação do minimizador global em $80 \%$ dos problemas se considerarmos como tempo máximo 5 vezes o melhor tempo encontrado para cada problema.

Utilizando esta formulação, os métodos foram comparados com relação ao número de iterações e o tempo necessário para encontrar uma boa aproximação de um minimizador global. Uma formulação alternativa, é apresentada no Apêndice C.

Na Figura (5.1) apresentamos o gráfico de desempenho dos métodos em relação ao número de iterações necessárias para a resolução dos problemas. Na Tabela 5.6 analisamos os dados do gráfico quanto a sua eficiência, ou seja, valores alcançados por cada método para $T_{1, j}$, e robustez, que verifica quantos problemas cada método conseguiu resolver independente do número de iterações. Os dados mostram um desempenho inicial muito superior do GRASP onde, em particular, o algoritmo C-GRA-GEN mostrou ser o algoritmo mais rápido em $88 \%$ dos problemas testados. Os demais métodos não chegaram a atingir a marca de $50 \%$. Com relação a robustez, o método de Tunneling, em sua versão Lis Seq, foi o único a conseguir resolver todos os problemas testados.

\begin{tabular}{|l|r|r|r|r|r|r|r|r|}
\hline & MST & RL(1) & RL(2) & RL(4) & LIS Orig & LIS Seq & GRA-GEN & C-GRA-GEN \\
\hline \hline Eficiência & 0.42 & 0.42 & 0.42 & 0.42 & 0.44 & 0.46 & 0.80 & 0.88 \\
Robustez & 0.94 & 0.96 & 0.96 & 0.94 & 0.98 & 1.00 & 0.98 & 0.98 \\
\hline
\end{tabular}

Tabela 5.6: Comparativo entre eficiência e robustez dos resultados apresentados no performance profile.

A Figura (5.2) mostra o gráfico de desempenho dos métodos em relação ao tempo gasto para a resolução dos problemas. Na Tabela 5.7 analisamos os dados do gráfico quanto a sua eficiência e robustez. A comparação de tempo mostra que, apesar do GRASP ter sido o método mais eficiente, a diferença de seu desempenho com os demais métodos caiu significativamente em relação a comparação anterior de número de iterações. Isto mostra que, apesar da Etapa de Construção do GRASP ter sido muito eficiente em encontrar pontos próximos a vizinhança de um minimizador global, utilizou para isto um tempo considerável de processamento. Em segundo lugar, em termos de desempenho, ficou o método Random Linkage tendo sido um dos métodos 

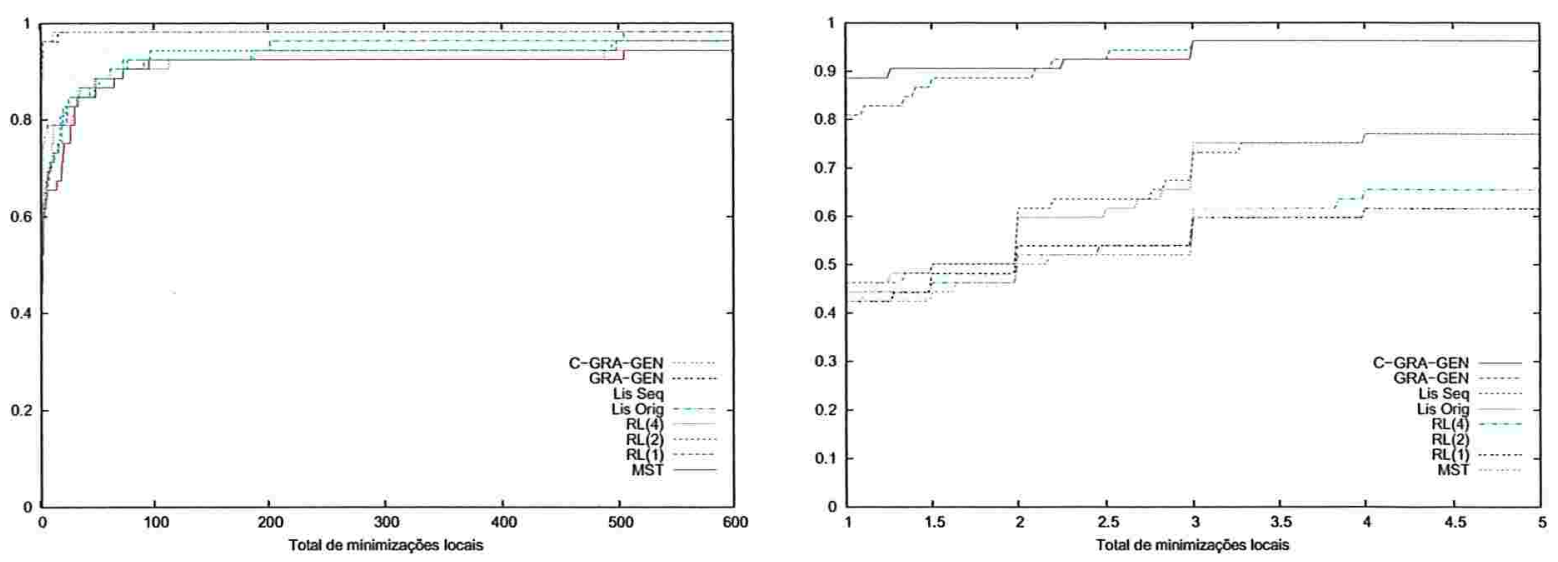

Figura 5.1: Gráfico de desempenho do número de minimizações locais realizadas pelos algoritmos para um conjunto de 52 problemas.

mais rápidos em $73 \%$ dos problemas analisados, para a sua versão com $\sigma=1$. Por último, apesar da sua robustez, o método de Tunneling mostrou ser o menos eficiente dos métodos analisados. De forma análoga ao GRASP, o método de Tunneling também apresentou uma sensível queda de desempenho entre entre os gráficos de performance de iteração e tempo, explicado pelo alto tempo consumido por sua Etapa de Construção.
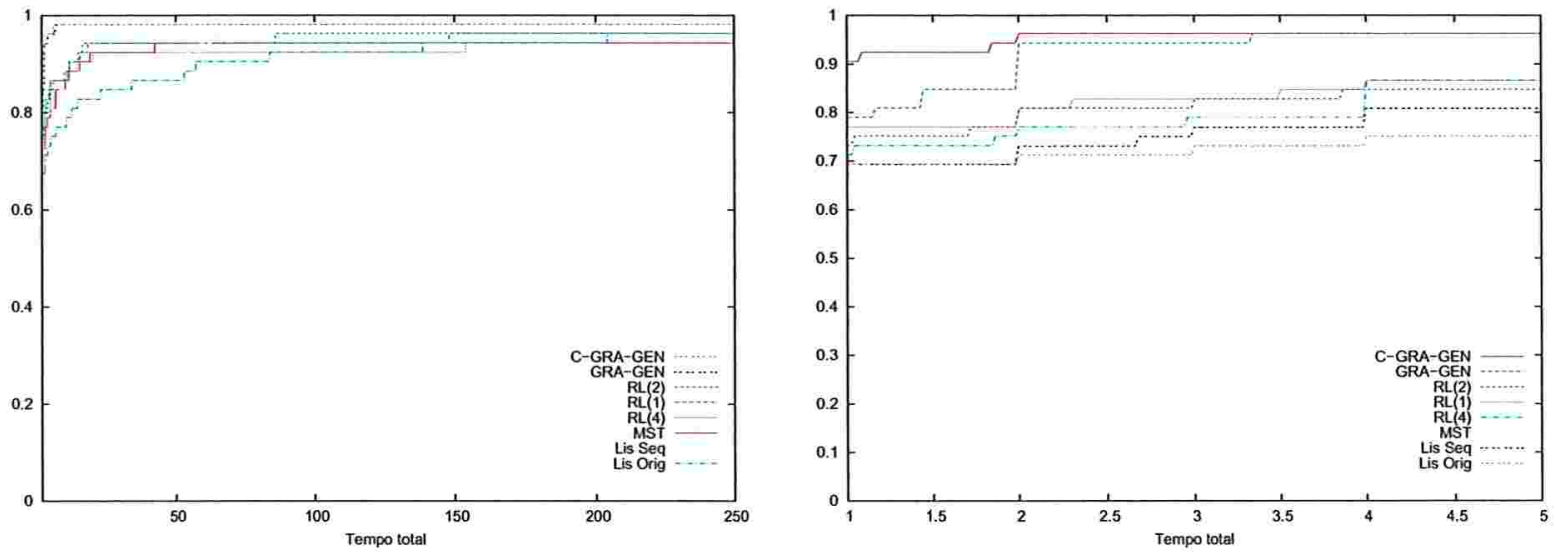

Figura 5.2: Gráfico de desempenho do tempo gasto pelos algoritmos para um conjunto de 52 problemas. 


\begin{tabular}{|l|r|r|r|r|r|r|r|r|}
\hline & MST & RL(1) & RL(2) & RL(4) & LIS Orig & LIS Seq & GRA-GEN & C-GRA-GEN \\
\hline \hline Eficiência & 0.67 & 0.76 & 0.73 & 0.71 & 0.69 & 0.69 & 0.78 & 0.90 \\
Robustez & 0.94 & 0.96 & 0.96 & 0.94 & 0.98 & 1.00 & 0.98 & 0.98 \\
\hline
\end{tabular}

Tabela 5.7: Comparativo entre eficiência e robustez dos resultados apresentados no performance profile.

Os resultados dos testes mostram que os algoritmos C-GRASP-GENCAN, GRASP-GENCAN, Random Linkage ( $\sigma \in\{1,2\}$ ) e Multistart foram os que apresentaram, na média, melhor desempenho para o conjunto de testes realizados. Visando avaliar o desempenho dos métodos relacionados a estes algoritmos com um número maior de variáveis, apresentamos no próximo capítulo o resultado de novos experimentos computacionais realizados em problemas de empacotamento. Dentro de cada método escolhemos o algoritmo que obteve melhor desempenho em comparação aos seus pares, em particular, utilizamos nos testes de empacotamento os algoritmos C-GRASP-GENCAN, Random Linkage $(\sigma=1)$ e Multistart. 


\section{Capítulo 6}

\section{Experimentos com problemas de empacotamento}

\subsection{Introdução}

Os resultados obtidos no Capítulo 5 mostraram um melhor desempenho dos métodos Multistart, Random Linkage e GRASP para um conjunto de 52 problemas selecionados a partir literatura clássica de programação não linear. No entanto, a grande maioria dos testes realizados consistiu de problemas de pequeno porte. Por este motivo, analisamos neste capítulo, o desempenho destes métodos para problemas de empacotamento, caracterizados por possuírem um grande número de mínimos locais e apresentarem um número de variáveis sensivelmente maior que os problemas analisados anteriormente. Desta forma, foi possível avaliar o desempenho de suas etapas de construção em dimensões maiores e diante de um número grande de minimizadores locais.

Para a realização dos testes, adaptamos a versões dos métodos Multistart, Random Linkage $(\sigma=2.0)$ e C-GRASP, utilizados no Capítulo 5, para a resolução de problemas de empacotamento. O critério de parada para cada um dos testes realizados foi a execução de cada algoritmo por 15 minutos. Os experimentos foram realizados em ambiente Linux de 32 bits (Ubuntu 6.06), em um computador AMD K8 $1.8 \mathrm{GHZ}$ com $1 \mathrm{~GB}$ de RAM, utilizando o compilador GNU Fortran (GCC) 3.4.6 com o parâmetros de compilação -04.

\subsection{Definição do problema}

Para a realização dos experimentos, foram escolhidos os 21 cenários de testes indicados em [6] que apresentaram o maior número de círculos a serem empacotados. Todos os testes realizados consistiram em uma instância do problema descrito a seguir:

Sejam $p^{c}$ círculos de raio $r$ e uma caixa de dimensões $\left[0, d_{1}\right] \times\left[0, d_{2}\right]$. Deseja-se saber se é possível empacotar estes círculos de forma que a sua interseç̧ão, para quaisquer par de círculos 
$p^{i}$ e $p^{j}$ onde $i \neq j$ seja de no máximo um ponto, ou seja, que não haja sobreposições de círculos. Para dados $p^{c}, r, d_{1}$ e $d_{2}$, deseja-se encontrar os pontos (centros de círculos) $p^{1}, \ldots, p^{c} \in$ $\left[r, d_{1}-r\right]\left[r, d_{2}-r\right]$ que resolvem o problema:

$$
\begin{array}{cl}
\text { Minimizar } & \sum_{i \neq j} \max \left(0,(2 r)^{2}-\left\|p^{i}-p^{j}\right\|_{2}^{2}\right)^{2} \\
\text { sujeita a } & \\
& r \leq p_{1}^{i} \leq d_{1}-r, \mathrm{e} \\
& r \leq p_{2}^{i} \leq d_{2}-r, \text { para } i=1, \ldots, c .
\end{array}
$$

Os pontos $p^{1}, \ldots, p^{c}$ são os centros dos círculos sendo que $p_{1}^{i}$ e $p_{2}^{i}$ representam as coordenadas $x$ e $y$ de $p^{i} \in \Re^{2}, \quad i=1, \ldots, c$. Se o valor da função objetivo é ZERO então foi encontrado um ponto que é minimizador global de (6.1).

Para cada um dos testes realizados foram indicadas as dimensões da caixa e o raio do círculo a ser empacotado. A partir destas informações, cada algoritmo procurou encontrar, dentro do prazo de 15 minutos, o número máximo de círculos que cabiam na caixa.

Os resultados dos testes foram divididos em seções de acordo com o método testado. Por último, comparamos, de forma sintética, o desempenho dos métodos testados. Os resultados foram apresentados em tabelas compostas pelas seguintes colunas:

Problema: problema testado;

Dimensão: dimensões da caixa onde serão empacotados os círculos;

Raio: raio dos círculos a serem empacotados;

Núm círculos: número de círculos empacotados;

$i t_{x^{*}}$ : número de iterações gastas até encontrar um empacotamento perfeito para o maior número de círculos avaliado;

$i t_{x}$ : número de iterações gastas até encontrar o maior número de círculos que cabem na caixa;

$t_{x^{*}}$ : tempo, em segundos, até encontrar $i t_{x^{*}}$;

$t_{*}$ : tempo, em segundos, até encontrar $i t_{x} ;$

$f_{\text {eval }}$ : total de avaliações da função objetivo até encontrar $x^{*}$;

$g_{\text {eval }}:$ total de avaliações do vetor gradiente até encontrar $x^{*}$;

Qtd $x_{0}$ : total de pontos iniciais gerados até encontrar $x^{*}$. 


\subsection{Multistart}

O desempenho do método Multistart foi semelhante ao resultado apresentado em [6], indicativo de que a implementação do algoritmo está correta. Dos 21 testes realizados, o método obteve sucesso em encontrar o maior número conhecido de círculos empacotados para 21 problemas. No problema 1.4, no entanto, o algoritmo conseguiu empacotar 85 círculos contra 86 de [6]. Esta diferença explica-se pelo tempo levado para empacotar 86 círculos, que em [6] foi de 37108.39 segundos.

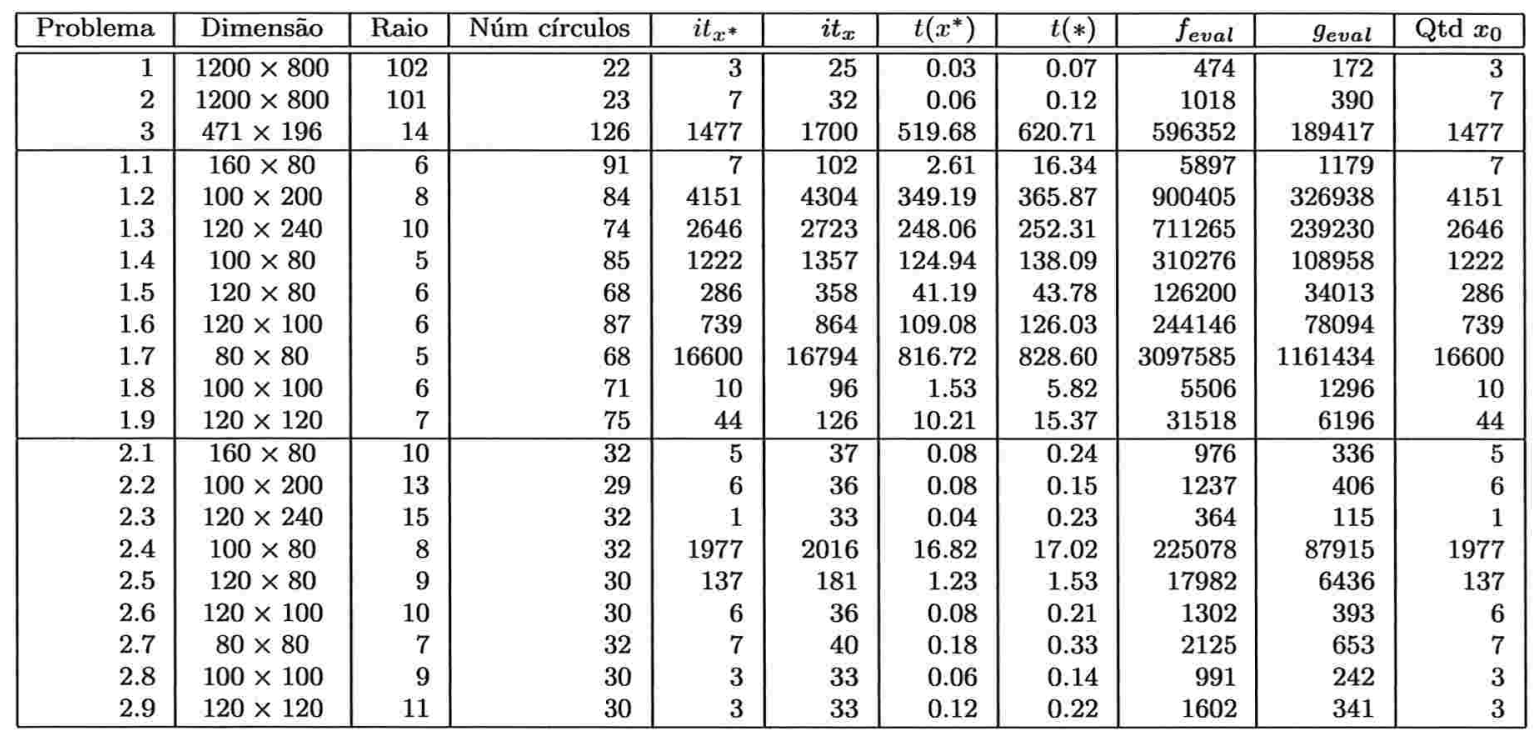

Tabela 6.1: Resultados obtidos a partir da versão implementada do Multistart, para o problema de empacotamento definido em (6.1).

\subsection{Random Linkage}

O método Random Linkage conseguiu empacotar a mesma quantidade de círculos que o $\mathrm{Mul}$ tistart em 20 problemas testados. Destes, o método conseguiu melhorar o desempenho do Multistart em 5 problemas. Como aspecto negativo, o método obteve desempenho muito inferior que o Multistart em 4 problemas. Isto ocorreu porque o método descartou pontos iniciais que resolveriam o problema de empacotamento.

\subsection{GRASP Contínuo}

Ao contrário do verificado no Capítulo 5, o C-GRASP obteve desempenho médio inferior aos demais métodos testados na resolução dos problemas de empacotamento. Em 9 dos problemas, 


\begin{tabular}{|c|c|c|c|c|c|c|c|c|c|c|}
\hline Problema & Dimensão & Raio & Núm círculos & $i t_{x^{*}}$ & $i t_{x}$ & $t\left(x^{*}\right)$ & $t(*)$ & $f_{\text {eval }}$ & $g_{\text {eval }}$ & Qtd $x_{0}$ \\
\hline 1 & $1200 \times 800$ & 102 & 22 & 3 & 25 & 0.03 & 0.08 & 477 & 172 & 3 \\
\hline 2 & $1200 \times 800$ & 101 & 23 & 7 & 32 & 0.07 & 0.12 & 1025 & 390 & 7 \\
\hline 3 & $471 \times 196$ & 14 & 126 & 1926 & 2217 & 721.40 & 888.14 & 954106 & 246903 & 193350 \\
\hline 1.1 & $160 \times 80$ & 6 & 91 & 22 & 115 & 9.20 & 17.93 & 20869 & 3761 & 1549 \\
\hline 1.2 & $100 \times 200$ & 8 & 84 & 1745 & 1883 & 177.01 & 190.26 & 555582 & 13762 & 175209 \\
\hline 1.3 & $120 \times 240$ & 10 & 74 & 4347 & 4422 & 429.45 & 433.28 & 1636167 & 396251 & 237105 \\
\hline 1.4 & $100 \times 80$ & 5 & 85 & 298 & 402 & 35.79 & 47.45 & 108280 & 26951 & 28970 \\
\hline 1.5 & $120 \times 80$ & 6 & 68 & 58 & 130 & 7.79 & 10.22 & 34307 & 7058 & 4987 \\
\hline 1.6 & $120 \times 100$ & 6 & 87 & 98 & 210 & 14.18 & 37.44 & 38709 & 9993 & 9134 \\
\hline 1.7 & $80 \times 80$ & 5 & 67 & 823 & 897 & 58.99 & 62.84 & 278193 & 67533 & 82424 \\
\hline 1.8 & $100 \times 100$ & 6 & 71 & 94 & 170 & 12.54 & 15.67 & 45832 & 10657 & 8851 \\
\hline 1.9 & $120 \times 120$ & 7 & 75 & 173 & 258 & 22.82 & 28.43 & 6728 & 1374 & 506 \\
\hline 2.1 & $160 \times 80$ & 10 & 32 & 5 & 37 & 0.08 & 0.24 & 981 & 336 & 5 \\
\hline 2.2 & $100 \times 200$ & 13 & 29 & 6 & 36 & 0.08 & 0.17 & 1243 & 406 & 6 \\
\hline 2.3 & $120 \times 240$ & 15 & 32 & 1 & 33 & 0.04 & 0.26 & 365 & 115 & 1 \\
\hline 2.4 & $100 \times 80$ & 8 & 32 & 1788 & 1827 & 17.42 & 17.69 & 206454 & 79596 & 1977 \\
\hline 2.5 & $120 \times 80$ & 9 & 30 & 128 & 172 & 1.21 & 1.51 & 17089 & 6020 & 137 \\
\hline 2.6 & $120 \times 100$ & 10 & 30 & 6 & 36 & 0.08 & 0.21 & 1308 & 393 & 6 \\
\hline 2.7 & $80 \times 80$ & 7 & 32 & 7 & 40 & 0.18 & 0.34 & 2132 & 653 & 7 \\
\hline 2.8 & $100 \times 100$ & 9 & 30 & 3 & 33 & 0.06 & 0.15 & 994 & 242 & 3 \\
\hline 2.9 & $120 \times 120$ & 11 & 30 & 3 & 33 & 0.12 & 0.22 & 1605 & 341 & 3 \\
\hline
\end{tabular}

Tabela 6.2: Resultados obtidos a partir da versão implementada do Random Linkage, com a constante $\sigma=1$, para o problema de empacotamento definido em (6.1).

não conseguiu empacotar a mesma quantidade que os demais métodos e, nos testes restantes, o C-GRASP conseguiu empacotar a quantidade esperada de círculos mais rapidamente apenas nos problemas 1.7 e 2.4. A perda de eficiência do algoritmo explica-se pela demora na execução de sua Etapa de Construção com o aumento do número de círculos a serem empacotados. No problema 1.1, por exemplo, foram gastos na Etapa de Construção, com passo $h=1.0,54.44$ segundos contra 0.17 da Etapa de Refinamento para empacotar 83 círculos. 


\begin{tabular}{|c|c|c|c|c|c|c|c|c|c|c|}
\hline Problema & Dimensão & Raio & Núm círculos & $i t_{x^{*}}$ & $i t_{x}$ & $t\left(x^{*}\right)$ & $t(*)$ & $f_{\text {eval }}$ & $g_{\text {eval }}$ & Qtd $x_{0}$ \\
\hline 1 & $1200 \times 800$ & 102 & 22 & 5 & 27 & 4.78 & 15.07 & 1001240 & 114 & 1 \\
\hline 2 & $1200 \times 800$ & 101 & 23 & 68 & 91 & 81.83 & 94.41 & 15693143 & 1197 & 12 \\
\hline 3 & $471 \times 196$ & 14 & 72 & 1 & 73 & 75.77 & 900.00 & 1756298 & 3 & 1 \\
\hline 1.1 & $160 \times 80$ & 6 & 83 & 1 & 84 & 51.16 & 914.66 & 918933 & 97 & 1 \\
\hline 1.2 & $100 \times 200$ & 8 & 78 & 8 & 86 & 127.39 & 876.46 & 2564407 & 523 & 2 \\
\hline 1.3 & $120 \times 240$ & 10 & 72 & 12 & 84 & 170.32 & 791.56 & 3979477 & 160 & 3 \\
\hline 1.4 & $100 \times 80$ & 5 & 83 & 13 & 102 & 157.72 & 852.75 & 2782226 & 550 & 3 \\
\hline 1.5 & $120 \times 80$ & 6 & 67 & 8 & 82 & 48.23 & 318.70 & 1268398 & 736 & 2 \\
\hline 1.6 & $120 \times 100$ & 6 & 84 & 1 & 85 & 49.96 & 916.41 & 870709 & 105 & 1 \\
\hline 1.7 & $80 \times 80$ & 5 & 68 & 28 & 104 & 127.39 & 373.97 & 3078903 & 530 & 5 \\
\hline 1.8 & $100 \times 100$ & 6 & 70 & 11 & 81 & 82.64 & 383.77 & 2046718 & 344 & 3 \\
\hline 1.9 & $120 \times 120$ & 7 & 74 & 13 & 87 & 144.49 & 651.28 & 2897795 & 712 & 3 \\
\hline 2.1 & $160 \times 80$ & 10 & 32 & 45 & 82 & 21.83 & 32.09 & 2188754 & 511 & 8 \\
\hline 2.2 & $100 \times 200$ & 13 & 29 & 10 & 43 & 4.33 & 11.04 & 551503 & 246 & 2 \\
\hline 2.3 & $120 \times 240$ & 15 & 32 & 19 & 57 & 12.86 & 27.34 & 1342143 & 265 & 4 \\
\hline 2.4 & $100 \times 80$ & 8 & 32 & 14 & 85 & 4.84 & 21.39 & 496848 & 224 & 3 \\
\hline 2.5 & $120 \times 80$ & 9 & 30 & 28 & 88 & 9.26 & 21.90 & 1059352 & 251 & 6 \\
\hline 2.6 & $120 \times 100$ & 10 & 30 & 153 & 183 & 47.36 & 52.44 & 5611915 & 1326 & 28 \\
\hline 2.7 & $80 \times 80$ & 7 & 32 & 2 & 34 & 1.03 & 6.18 & 103000 & 198 & 1 \\
\hline 2.8 & $100 \times 100$ & 9 & 30 & 1 & 31 & .81 & 5.74 & 92682 & 68 & 1 \\
\hline 2.9 & $120 \times 120$ & 11 & 30 & 2 & 38 & 1.41 & 9.06 & 167541 & 95 & 1 \\
\hline
\end{tabular}

Tabela 6.3: Resultados obtidos a partir da versão implementada do C-GRASP para o problema de empacotamento definido em (6.1). 


\subsection{Comparativo dos resultados}

Analisando o resultados dos testes, observamos que o Multitart foi o único método a empacotar o número de círculos esperado em todos os problemas testados. Na Tabela 6.4 verificamos que, dentre os 12 problemas nos quais o Multistart e o C-GRASP empacotaram o mesmo número de círculos, o Multistart encontrou a solução esperada em um número menor de iterações em 7 problemas contra 5 do C-GRASP. Neste critério, o desempenho do Random Linkage foi melhor em 6 problemas contra 5 do Multistart. Verificamos ainda, na Tabela 6.5, que o C-GRASP encontrou a solução esperada mais rapidamente apenas no teste 1.7. O motivo desta divergência entre o seu desempenho no número de iterações com o tempo de processamento deve-se ao tempo gasto pela Etapa de Construção do método em encontrar um boa aproximação do minimizador global. O Random Linkage, por sua vez, saiu-se melhor em 6 problemas contra 6 do Multistart. Nos demais problemas o desempenho de tempo dos dois métodos foi semelhante. Por último, apresentamos nas Figuras 6.1-6.21 a solução final devolvida por cada método para cada um dos problemas testados.

A comparação dos resultados mostra um desempenho ligeiramente superior do Multistart em relação ao Random Linkage. Em particular, o Random Linkage saiu-se melhor em problemas que o Multistart teve dificuldades em encontrar um empacotamento. Isto mostra que o Random Linkage obteve êxito, em vários dos problemas, em eliminar pontos iniciais desnecessários.

\begin{tabular}{|r|r|r|r|r|r|r|}
\hline & \multicolumn{3}{|c|}{$i t\left(x^{*}\right)$} & \multicolumn{3}{|c|}{$i t(*)$} \\
\hline Problema & Multistart & R.Linkage & C-GRASP & Multistart & R.Linkage & C-GRASP \\
\hline 1 & 3 & 3 & 5 & 25 & 25 & 27 \\
2 & 7 & 7 & 68 & 32 & 32 & 91 \\
3 & 1477 & 1926 & - & 1700 & 2217 & - \\
\hline 1.1 & 7 & 22 & - & 102 & 115 & - \\
1.2 & 4151 & 1745 & - & 4304 & 1883 & - \\
1.3 & 2646 & 4347 & - & 2723 & 4422 & - \\
1.4 & 1222 & 298 & - & 1357 & 402 & - \\
1.5 & 286 & 58 & - & 358 & 130 & - \\
1.6 & 739 & 98 & - & 864 & 210 & - \\
1.7 & 16600 & - & 28 & 16794 & - & 104 \\
1.8 & 10 & 94 & - & 96 & 170 & - \\
1.9 & 44 & 173 & - & 126 & 258 & - \\
\hline 2.1 & 5 & 5 & 45 & 37 & 37 & 82 \\
2.2 & 6 & 6 & 10 & 36 & 36 & 43 \\
2.3 & 1 & 1 & 19 & 33 & 33 & 57 \\
2.4 & 1977 & 1788 & 14 & 2016 & 1827 & 85 \\
2.5 & 137 & 128 & 28 & 181 & 172 & 88 \\
2.6 & 6 & 6 & 153 & 36 & 36 & 33 \\
2.7 & 7 & 7 & 2 & 40 & 40 & 33 \\
2.8 & 3 & 3 & 3 & 33 & 33 & 38 \\
2.9 & & & & & & 33 \\
\hline
\end{tabular}

Tabela 6.4: Comparativo do número de iterações realizadas por cada um dos métodos na resolução do problema de empacotamento definido em (6.1). 


\begin{tabular}{|r|r|r|r|r|r|r|}
\hline & \multicolumn{3}{|c|}{$t\left(x^{*}\right)$} & \multicolumn{3}{|c|}{$t(*)$} \\
\hline Problema & Multistart & R.Linkage & C-GRASP & Multistart & R.Linkage & C-GRASP \\
\hline 1 & 0.03 & 0.03 & 4.78 & 0.07 & 0.08 & 15.07 \\
3 & 0.06 & 0.07 & 81.83 & 0.12 & 0.12 & 94.41 \\
\hline 1.1 & 519.68 & 721.40 & - & 620.71 & 888.14 & - \\
1.2 & 349.19 & 177.01 & - & 16.34 & 17.93 & - \\
1.3 & 248.06 & 429.45 & - & 365.87 & 190.26 & - \\
1.4 & 124.94 & 35.79 & - & 252.31 & 433.28 & - \\
1.5 & 41.19 & 7.79 & - & 138.09 & 47.45 & - \\
1.6 & 109.08 & 14.18 & - & 43.78 & 10.22 & - \\
1.7 & 816.72 & - & 127.39 & 828.60 & 37.44 & - \\
1.8 & 1.53 & 12.54 & - & 5.82 & 15.67 & 373.97 \\
1.9 & 10.21 & 22.82 & - & 15.37 & 28.43 & - \\
2.1 & 0.08 & 0.08 & 21.83 & 0.24 & 0.24 & - \\
2.2 & 0.08 & 0.08 & 4.33 & 0.15 & 0.17 & 11.04 \\
2.3 & 0.04 & 0.04 & 12.86 & 0.23 & 0.26 & 27.34 \\
2.4 & 16.82 & 17.42 & 4.84 & 17.02 & 17.69 & 21.39 \\
2.5 & 1.23 & 1.21 & 9.26 & 1.53 & 1.51 & 21.90 \\
2.6 & 0.08 & 0.08 & 47.36 & 0.21 & 0.21 & 52.44 \\
2.7 & 0.18 & 0.18 & 1.03 & 0.33 & 0.34 & 6.18 \\
2.8 & 0.06 & 0.06 & 0.81 & 0.14 & 0.15 & 5.74 \\
2.9 & 0.12 & 0.12 & 1.41 & 0.22 & 0.22 & 9.06 \\
\hline
\end{tabular}

Tabela 6.5: Comparativo do tempo demorado por cada um dos métodos na resolução do problema de empacotamento definido em (6.1). 


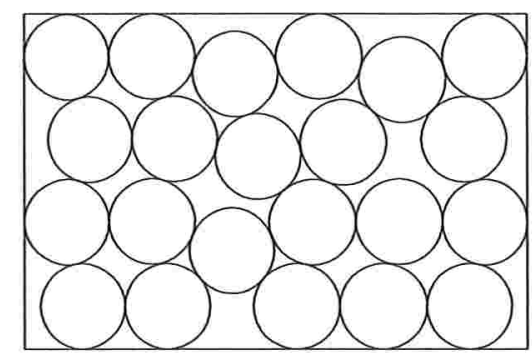

Multistart

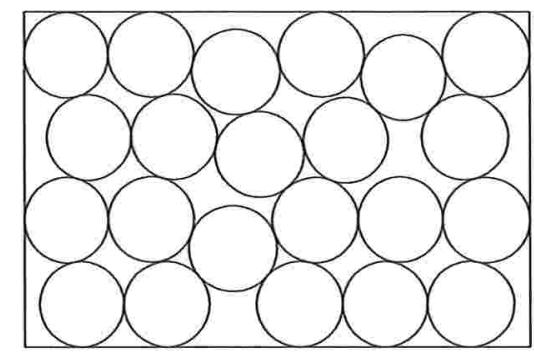

Random Linkage

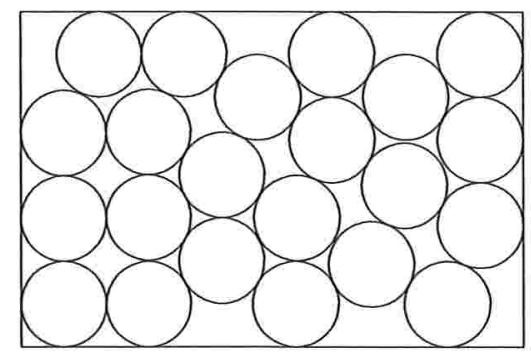

C-GRASP

Figura 6.1: Resultado final encontrado pelos métodos para o problema 1.

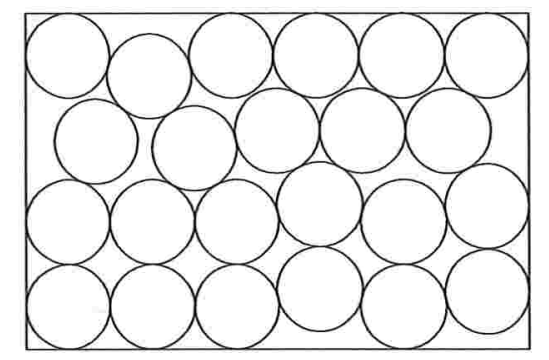

Multistart

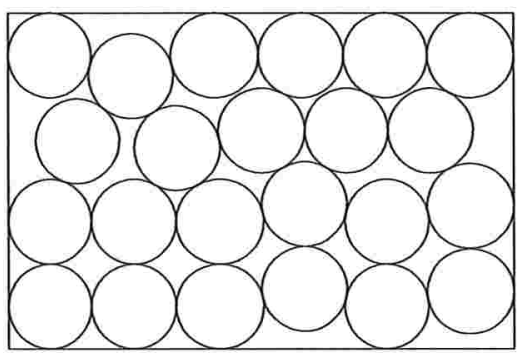

Random Linkage

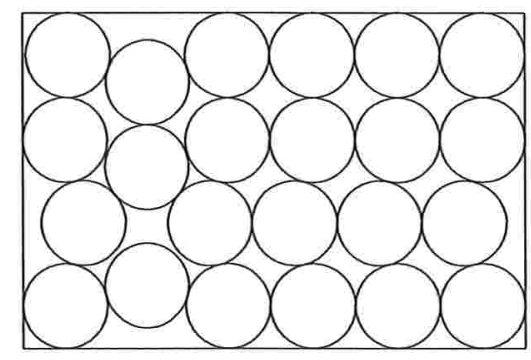

C-GRASP

Figura 6.2: Resultado final encontrado pelos métodos para o problema 2.

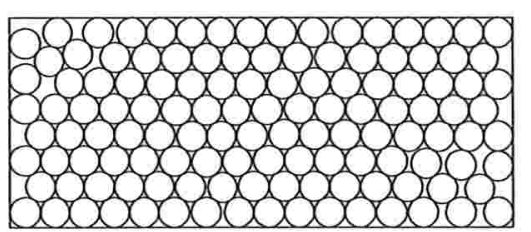

Multistart

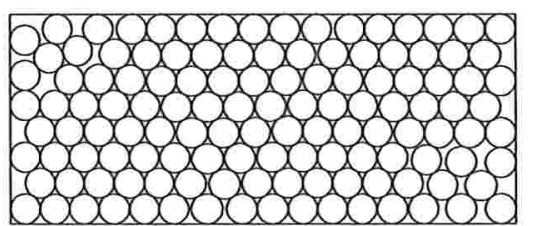

Random Linkage

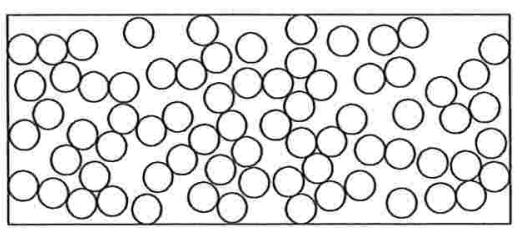

C-GRASP

Figura 6.3: Resultado final encontrado pelos métodos para o problema 3. 


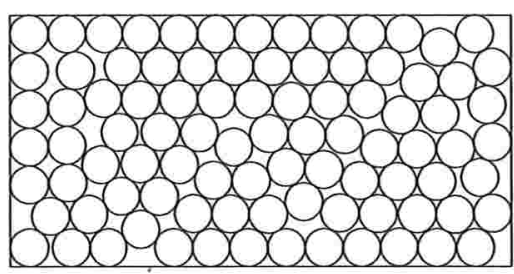

Multistart

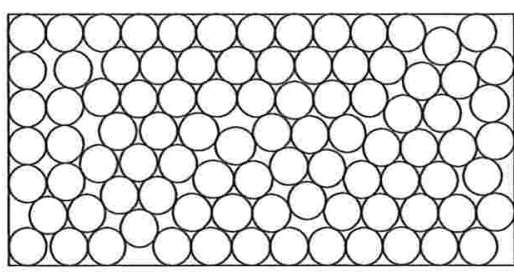

Random Linkage

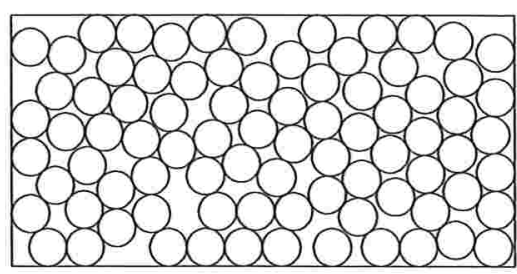

C-GRASP

Figura 6.4: Resultado final encontrado pelos métodos para o problema 1.1.

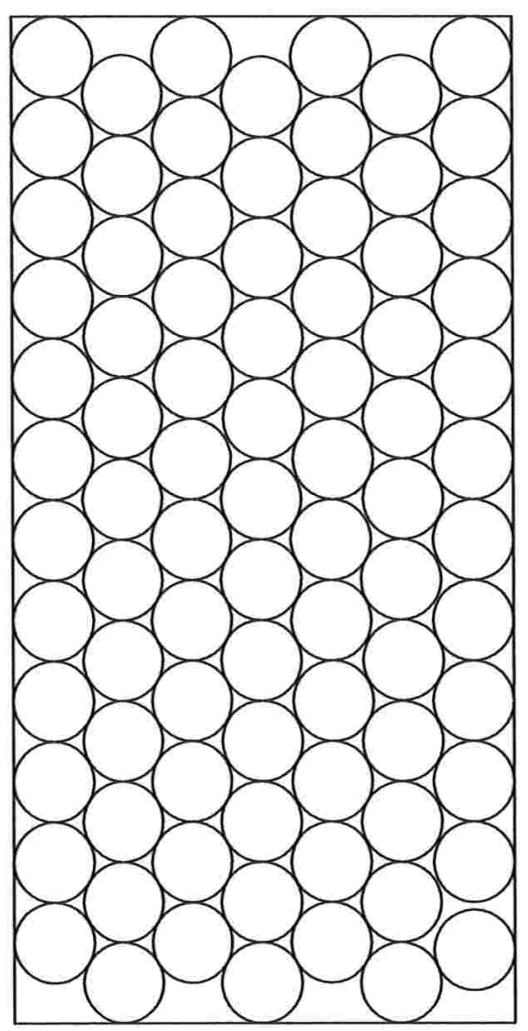

Multistart

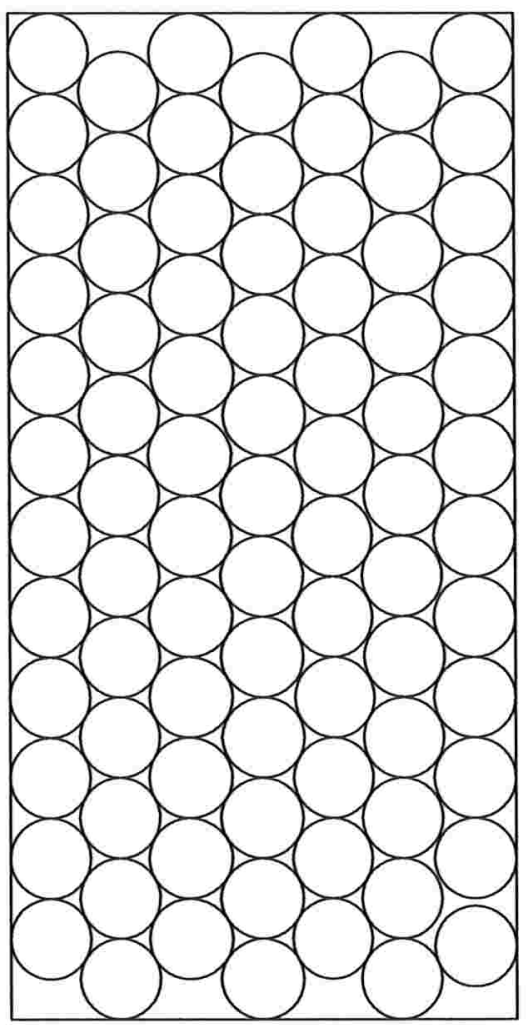

Random Linkage

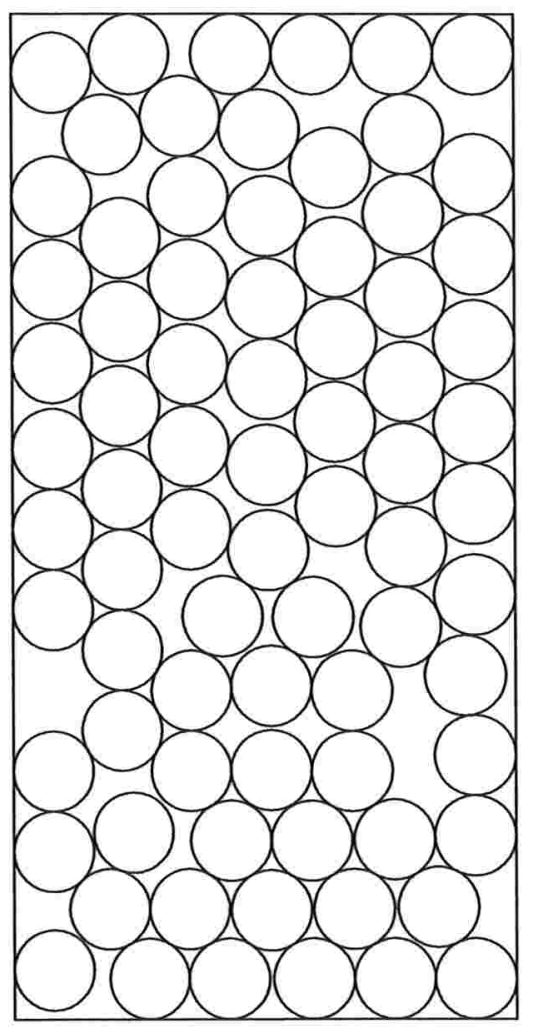

C-GRASP

Figura 6.5: Resultado final encontrado pelos métodos para o problema 1.2. 


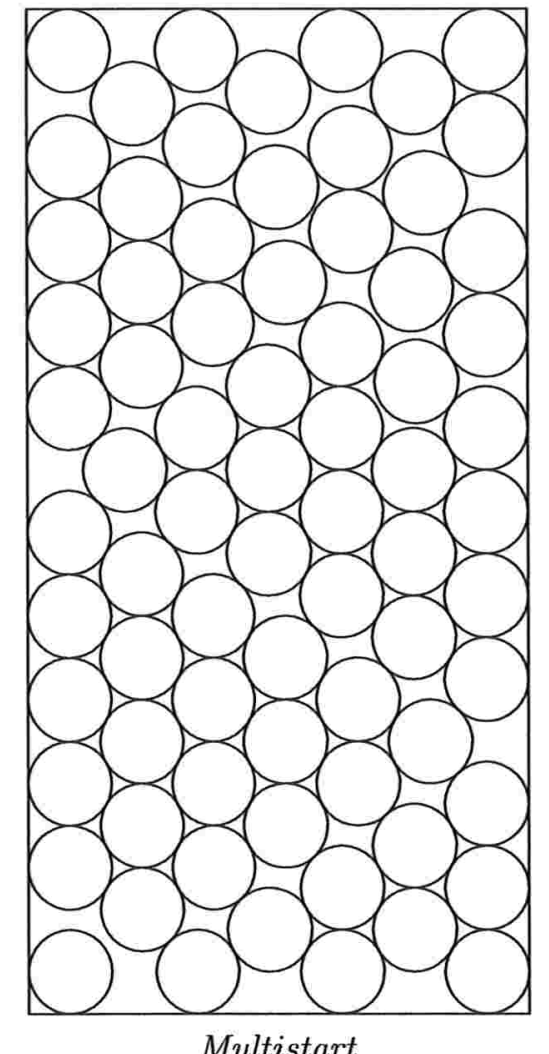

Multistart

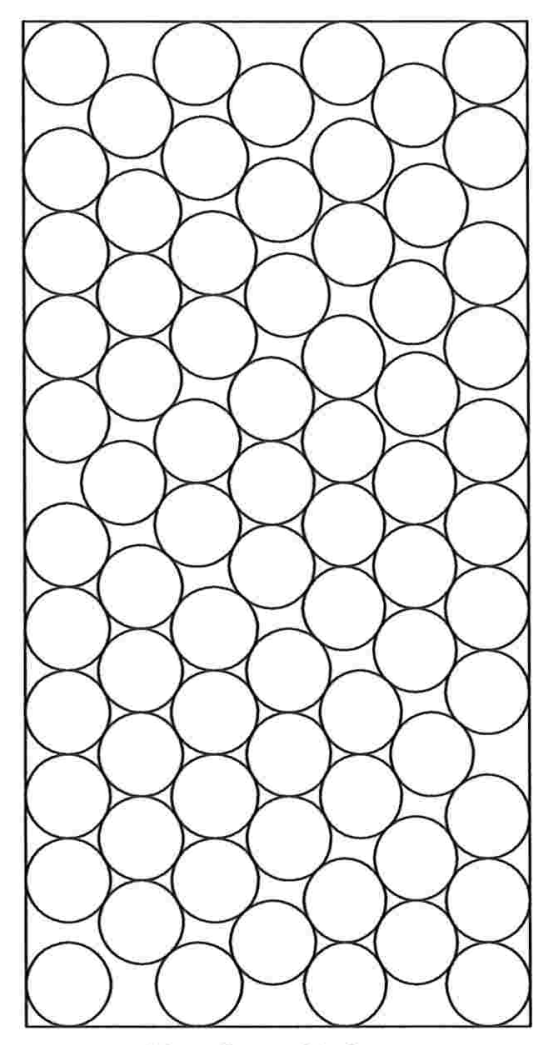

Random Linkage

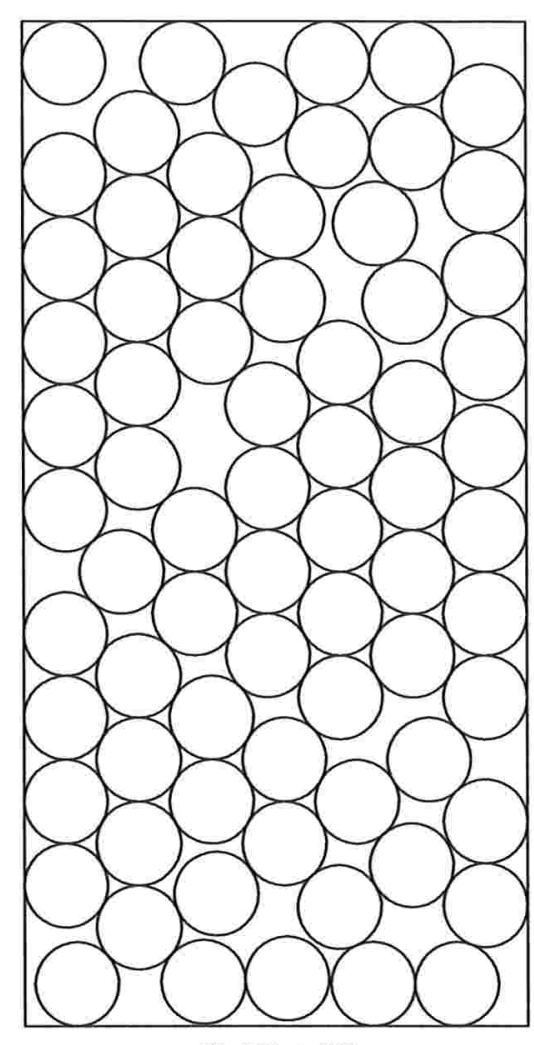

C-GRASP

Figura 6.6: Resultado final encontrado pelos métodos para o problema 1.3.

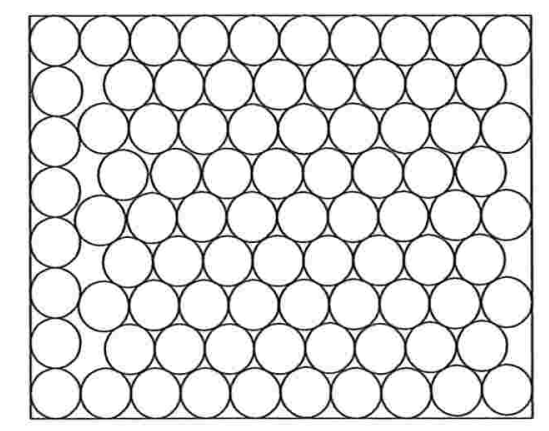

Multistart

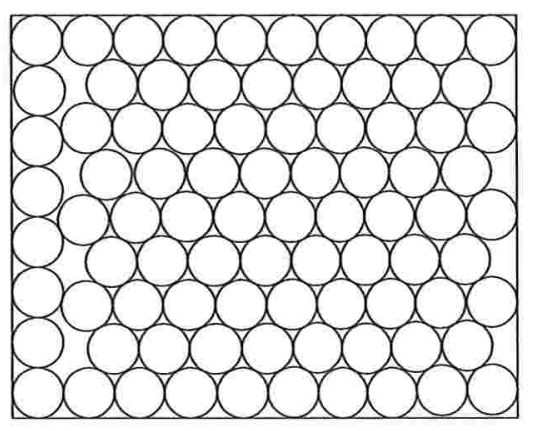

Random Linkage

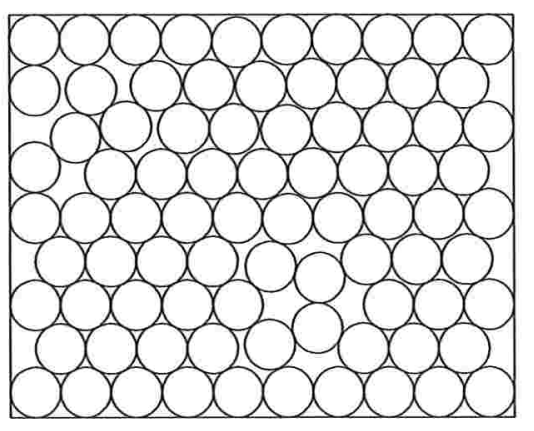

C-GRASP

Figura 6.7: Resultado final encontrado pelos métodos para o problema 1.4. 


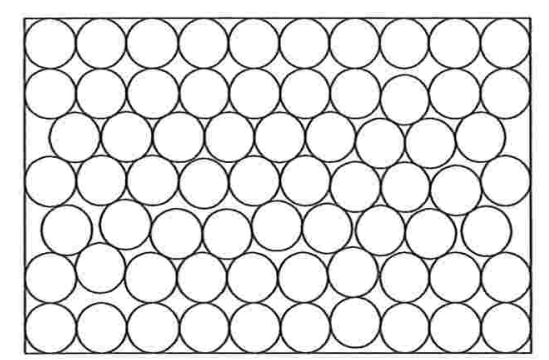

Multistart

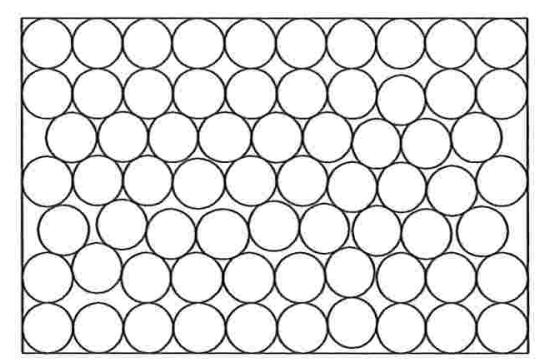

Random Linkage

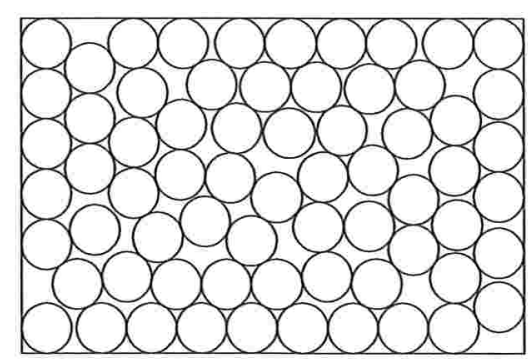

C-GRASP

Figura 6.8: Resultado final encontrado pelos métodos para o problema 1.5.

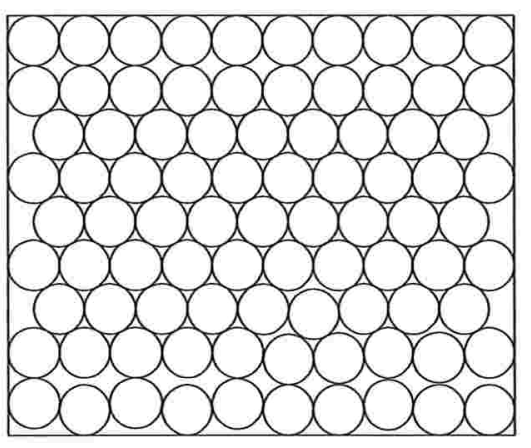

Multistart

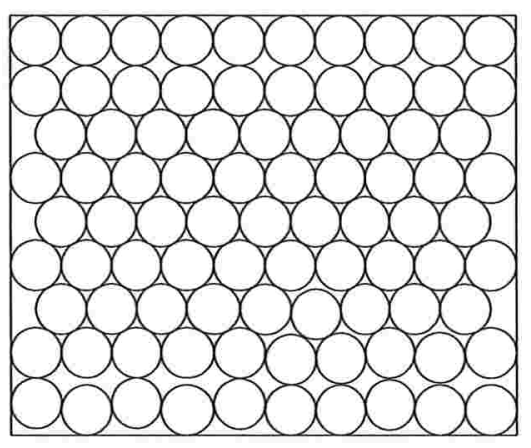

Random Linkage

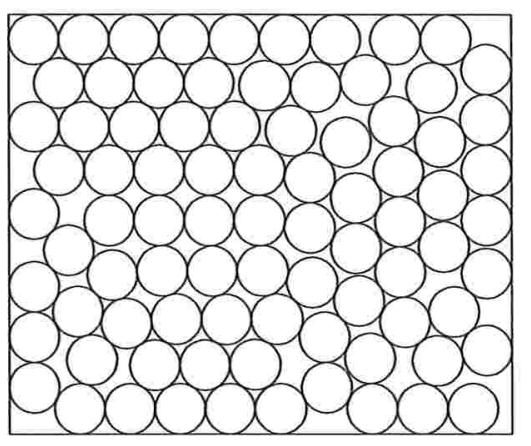

C-GRASP

Figura 6.9: Resultado final encontrado pelos métodos para o problema 1.6.

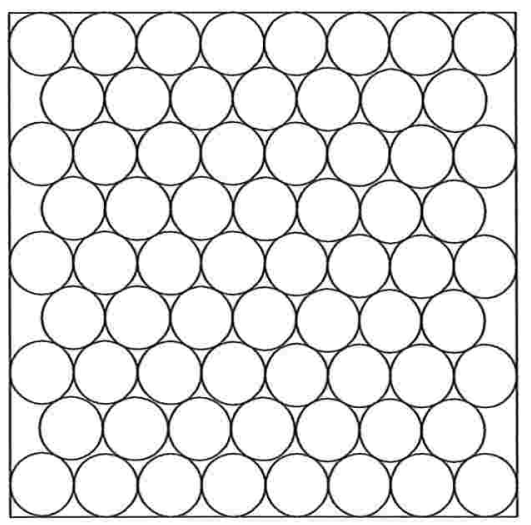

Multistart

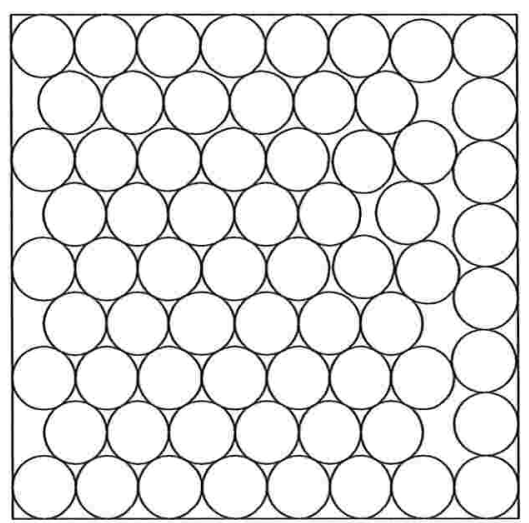

Random Linkage

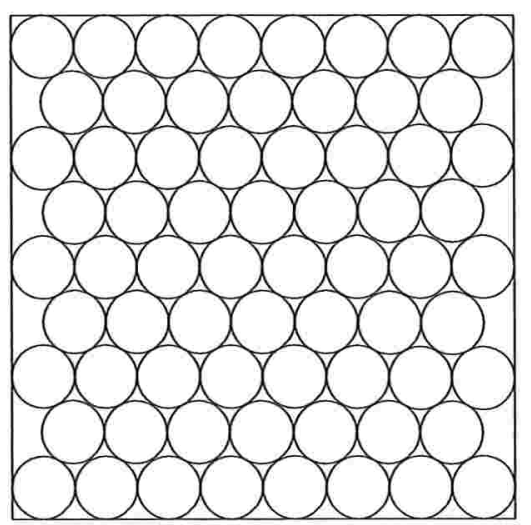

C-GRASP

Figura 6.10: Resultado final encontrado pelos métodos para o problema 1.7. 


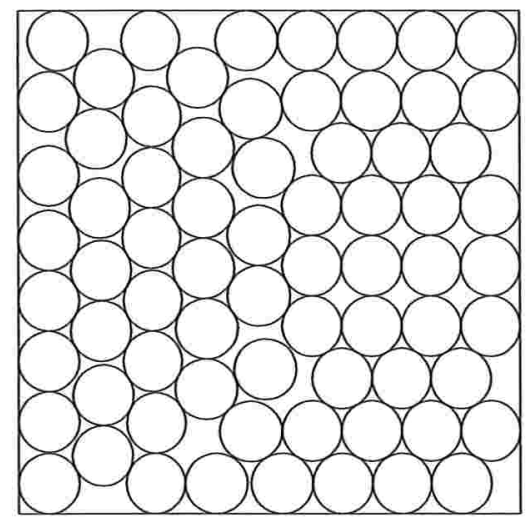

Multistart

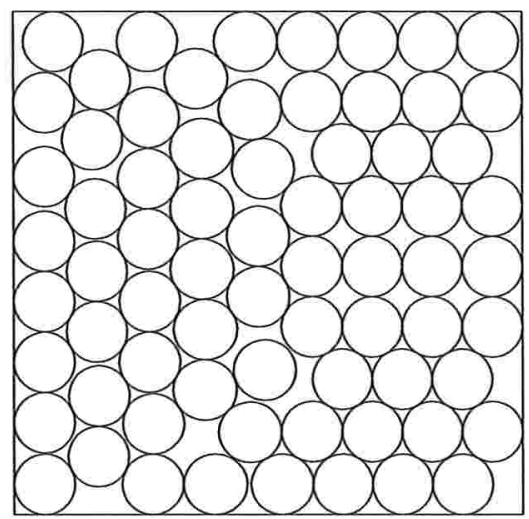

Random Linkage

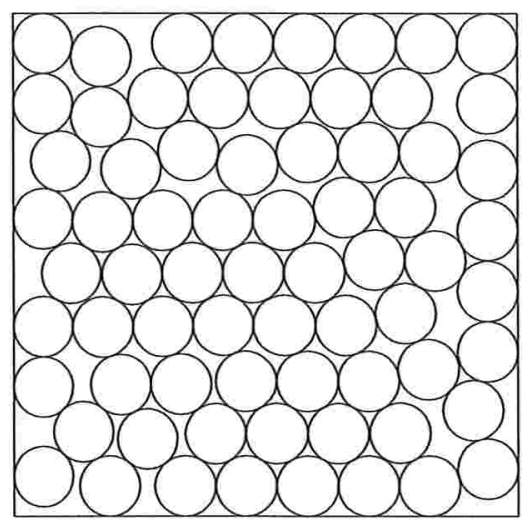

C-GRASP

Figura 6.11: Resultado final encontrado pelos métodos para o problema 1.8.

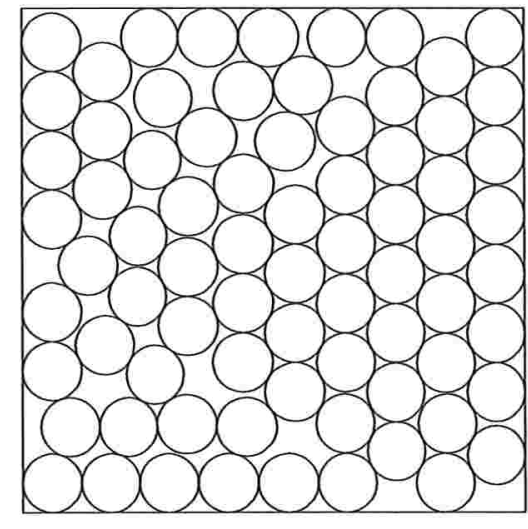

Multistart

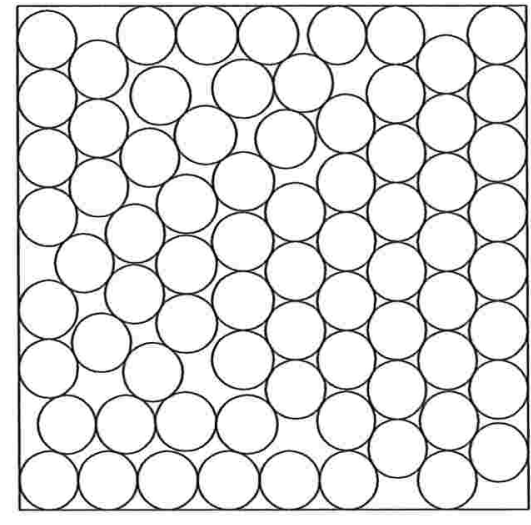

Random Linkage

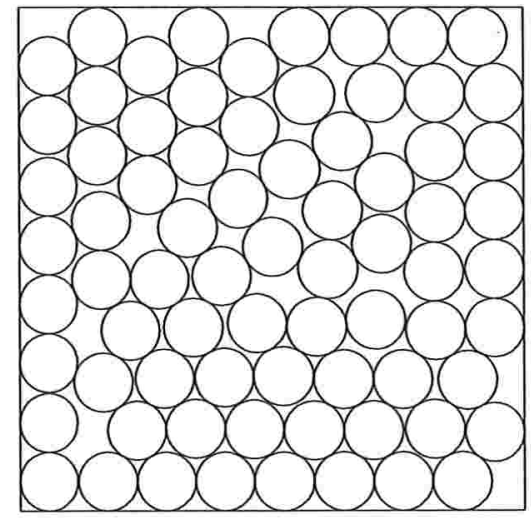

C-GRASP

Figura 6.12: Resultado final encontrado pelos métodos para o problema 1.9. 


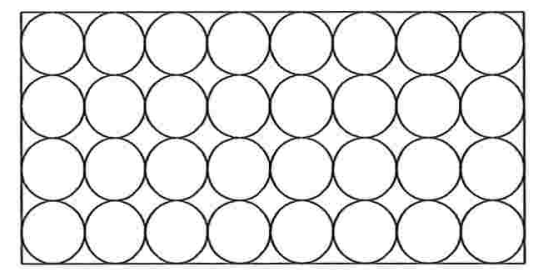

Multistart

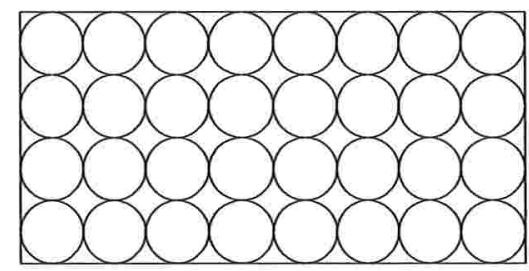

Random Linkage

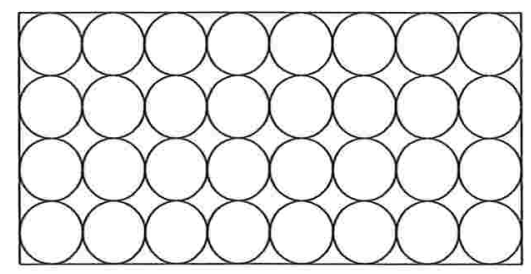

C-GRASP

Figura 6.13: Resultado final encontrado pelos métodos para o problema 2.1.

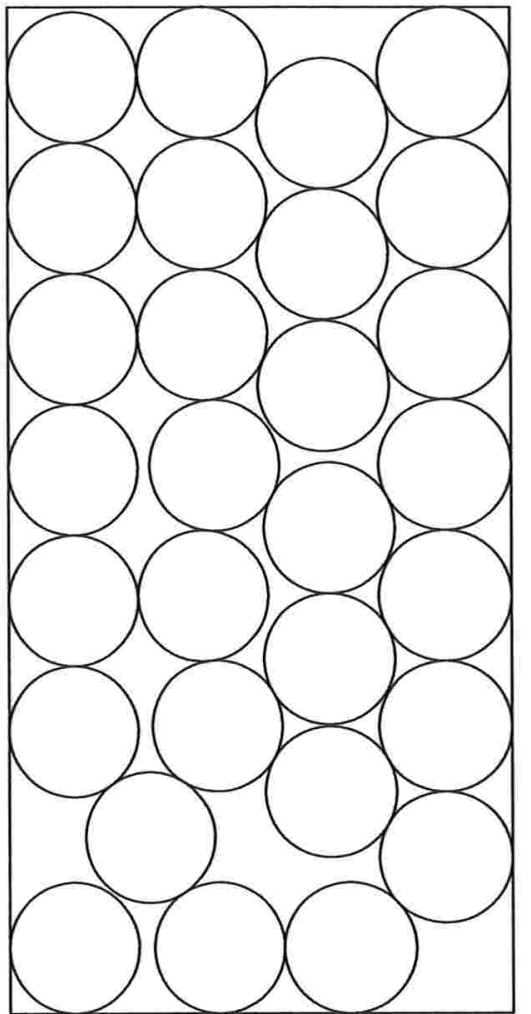

Multistart

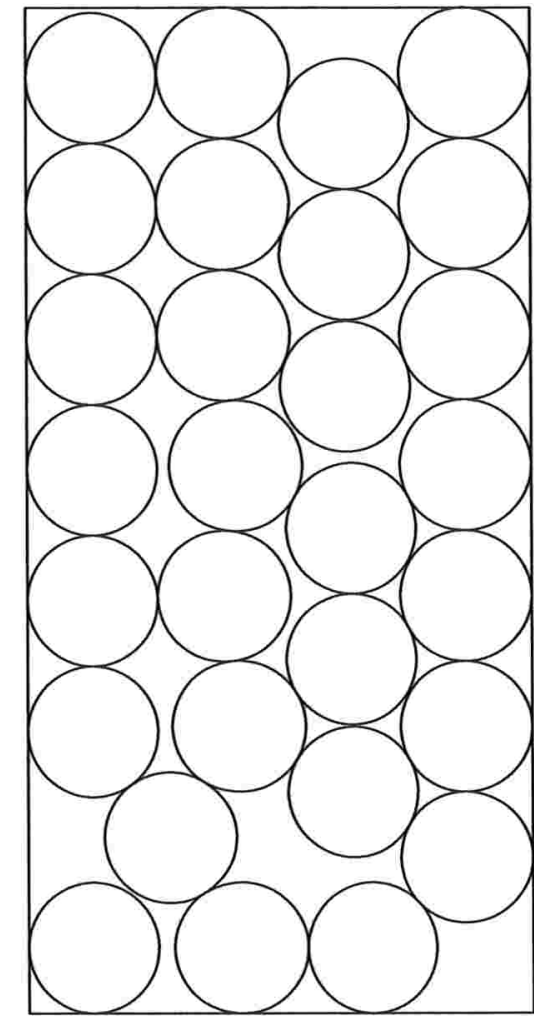

Random Linkage

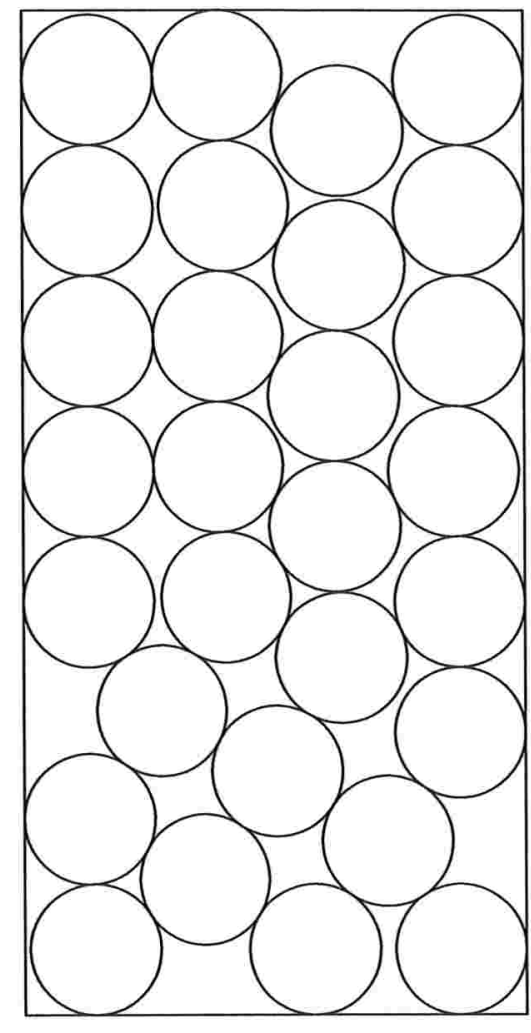

C-GRASP

Figura 6.14: Resultado final encontrado pelos métodos para o problema 2.2. 


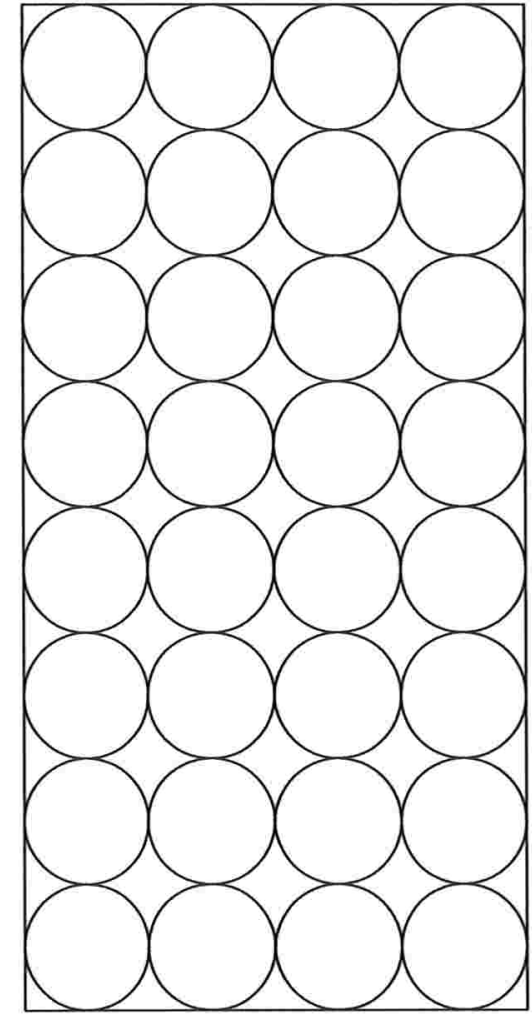

Multistart

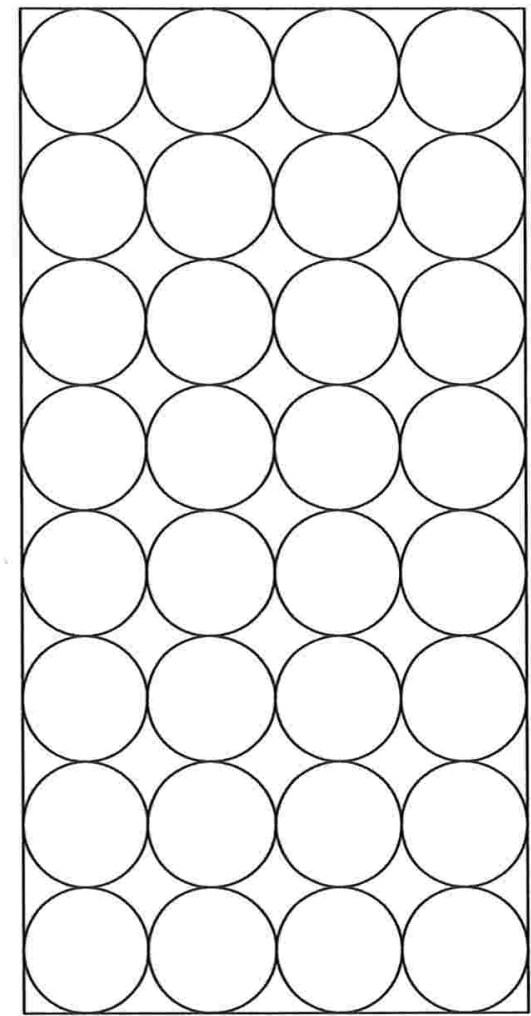

Random Linkage

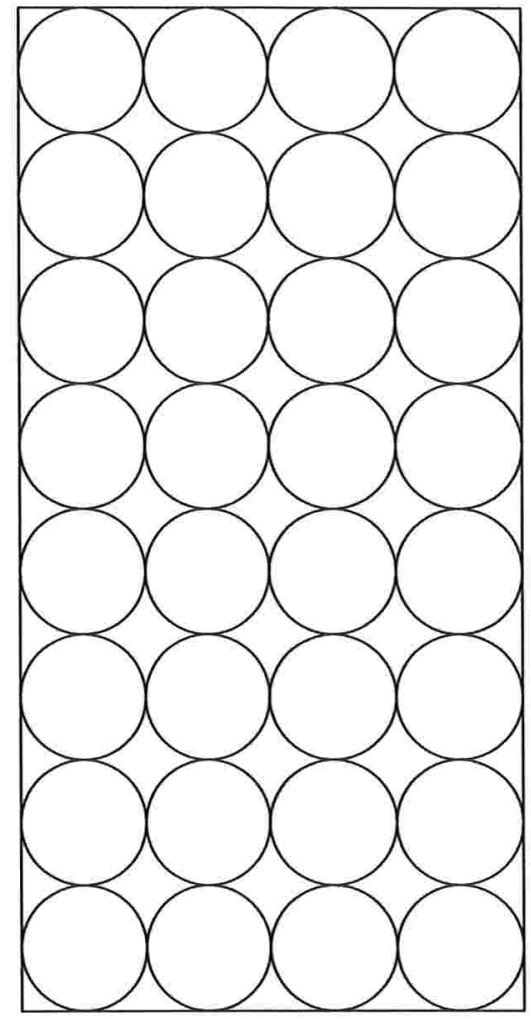

C-GRASP

Figura 6.15: Resultado final encontrado pelos métodos para o problema 2.3.

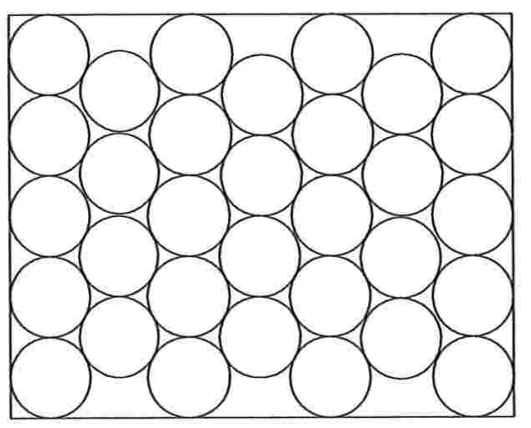

Multistart

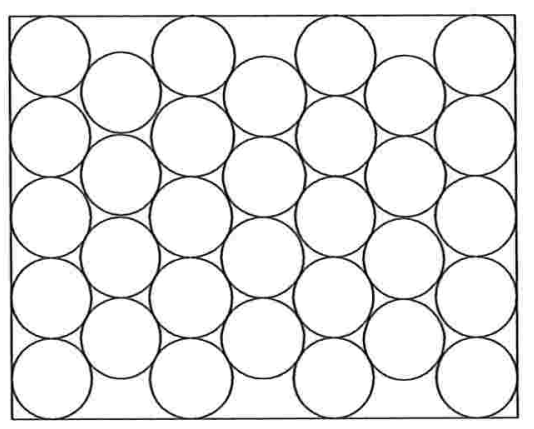

Random Linkage

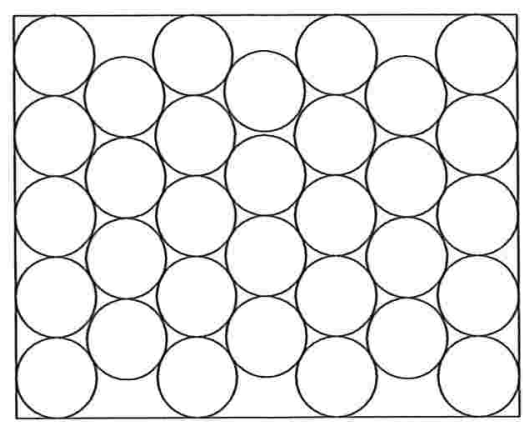

C-GRASP

Figura 6.16: Resultado final encontrado pelos métodos para o problema 2.4. 


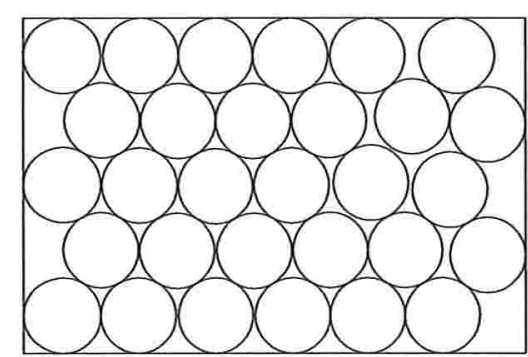

Multistart

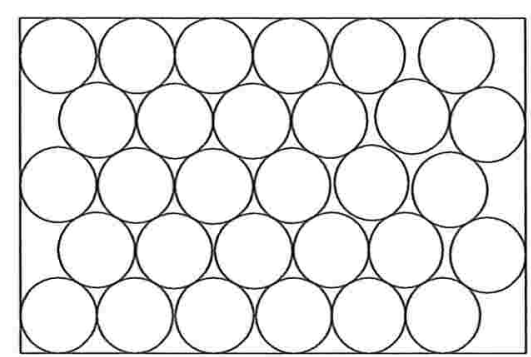

Random Linkage

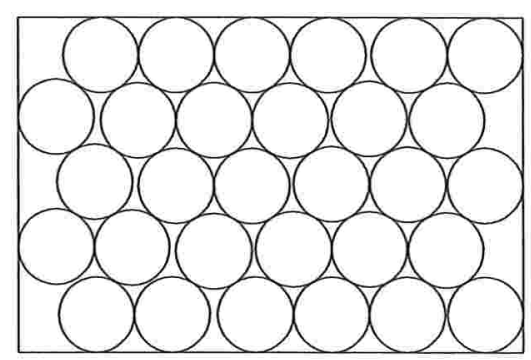

C-GRASP

Figura 6.17: Resultado final encontrado pelos métodos para o problema 2.5.

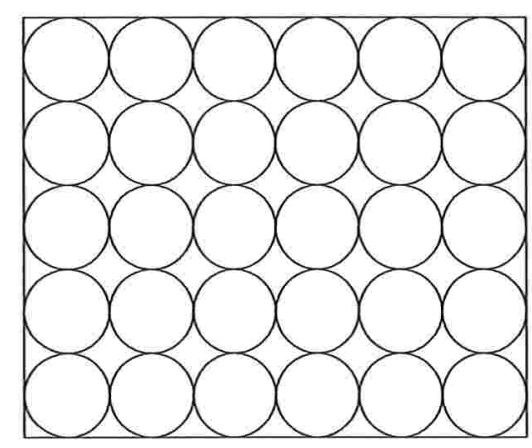

Multistart

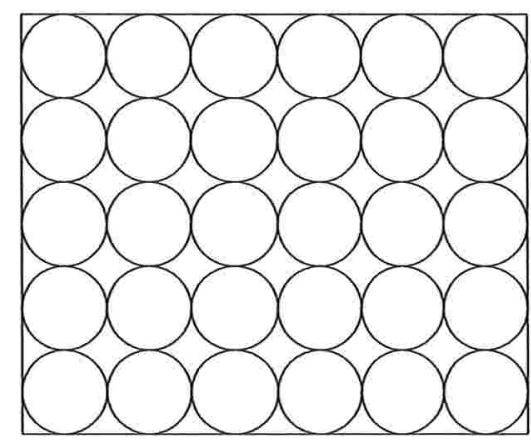

Random Linkage

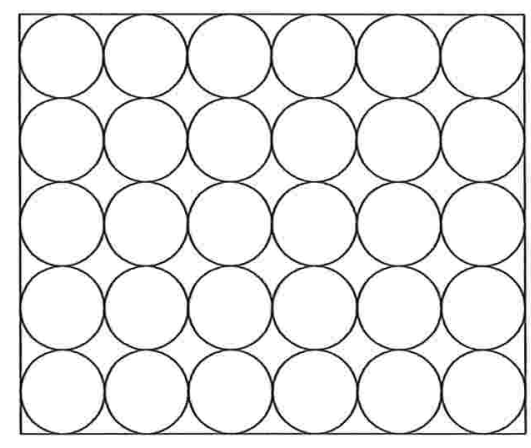

C-GRASP

Figura 6.18: Resultado final encontrado pelos métodos para o problema 2.6.

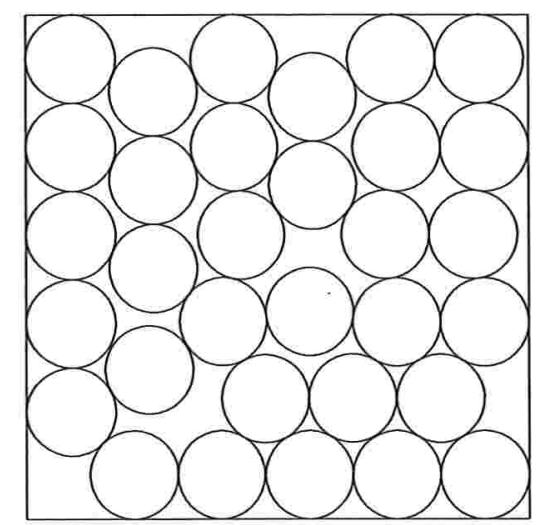

Multistart

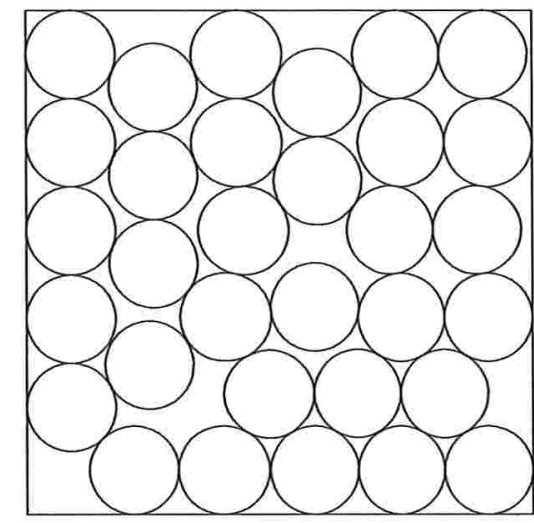

Random Linkage

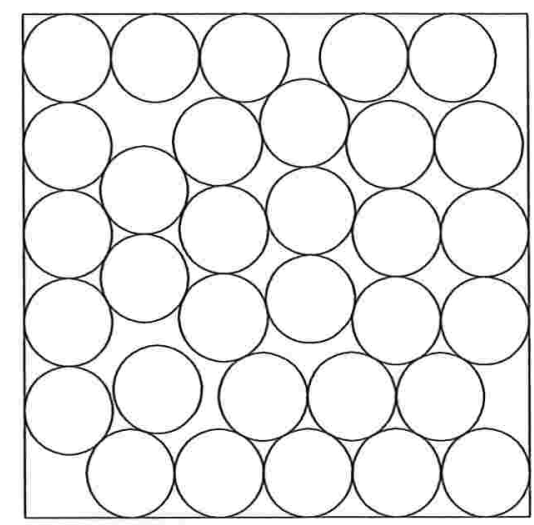

C-GRASP

Figura 6.19: Resultado final encontrado pelos métodos para o problema 2.7. 


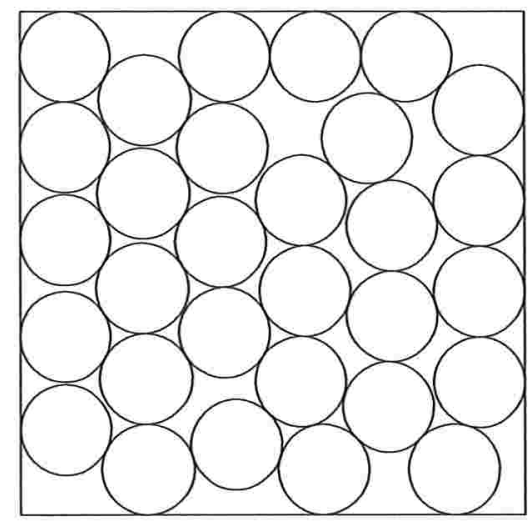

Multistart

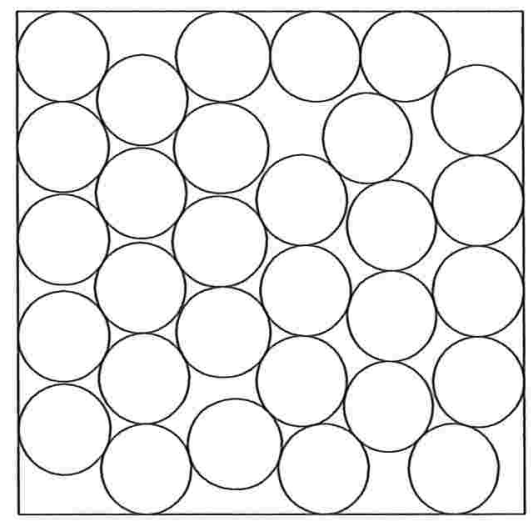

Random Linkage

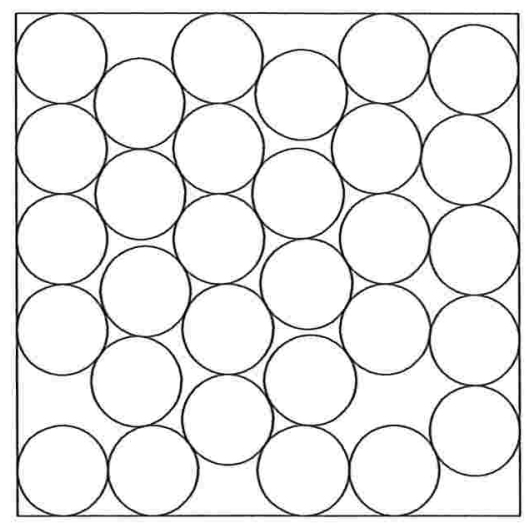

C-GRASP

Figura 6.20: Resultado final encontrado pelos métodos para o problema 2.8.

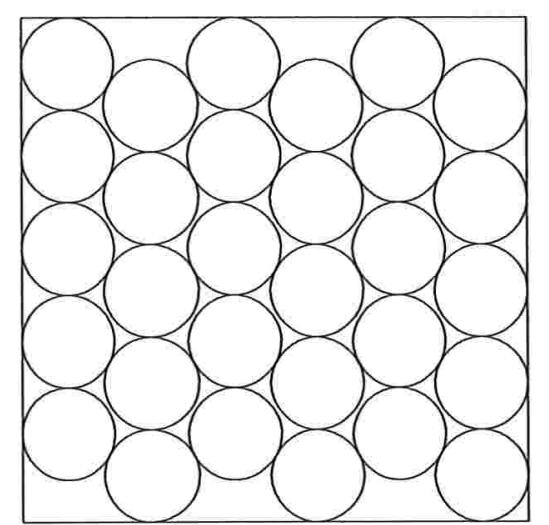

Multistart

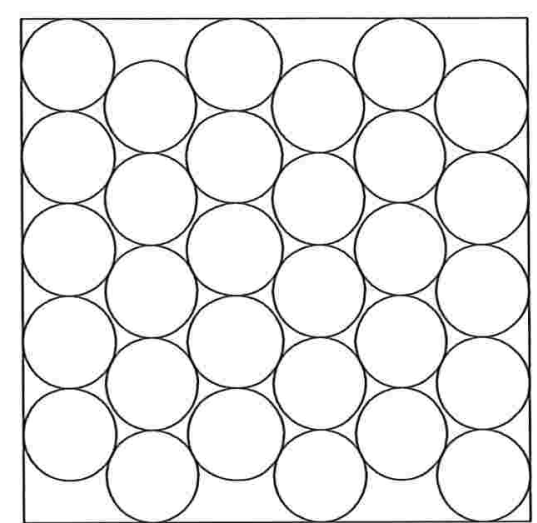

Random Linkage

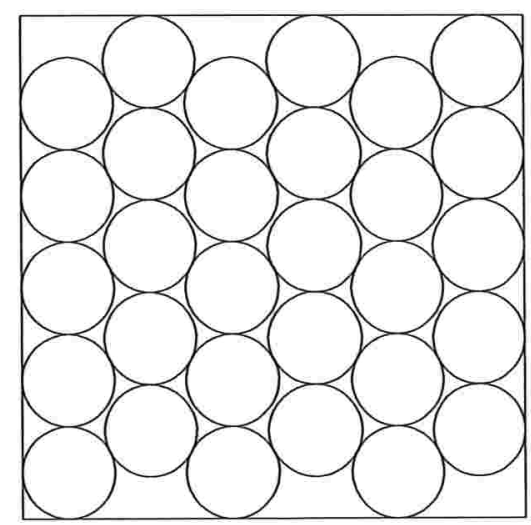

C-GRASP

Figura 6.21: Resultado final encontrado pelos métodos para o problema 2.9. 


\section{Capítulo 7}

\section{Conclusões}

Os experimentos realizados sobre os algoritmos estocásticos estudados nos permitiram avaliar em situações práticas diferenciadas os pontos fortes e fracos de cada um dos métodos. Em particular, a utilização comum do GENCAN na Etapa de Refinamento garantiu que o diferencial de cada método ocorresse na Etapa de Construção. Neste aspecto, utilizando o método Multistart como referencial destacamos os desempenho dos métodos para cada conjunto de problemas.

O método Random Linkage mostrou-se muito bom na resolução de problemas tanto de pequeno como de grande porte. Nos testes do Capítulo 5 foi o segundo método mais eficiente e nos testes do Capítulo 6 teve um desempenho muito bom, chegando a ser método mais eficiente para 6 problemas de empacotamento.

O método de Tunneling utilizando a curva de Lissajous mostrou-se eficiente para problemas com um número reduzido de variáveis aplicados a uma região factível pequena. Isto pode ser verificado pelo seu desempenho nos testes A.3, A.4, A.7, A.10, A.13, A.22, A.23, A.29, A.6, A.12. Por outro lado, analisando a função A.5 verificamos que o desempenho do método de Tunneling foi melhor que o Multistart apenas no teste com menor número de variáveis $(n=5)$ tendo o seu desempenho piorado com o aumento deste número $(n \in\{8,10,20,30\})$.

Por último, analisamos o desempenho do C-GRASP. Tendo sido o método mais eficiente nos testes realizados no Capítulo 5 não conseguiu repetir sua performance nos testes de empacotamento (Capítulo 6). Este resultado é explicado pelo tempo consumido pela sua Etapa de Construção que aumenta sensivelmente com o incremento do número de variáveis do problema.

A partir dos resultados apresentados pelos experimentos, não foi possível definir um método único na resolução dos problemas avaliados neste texto. Não obstante, destacamos a eficiência do C-GRASP na resolução dos problemas do Capítulo 5, sensivelmente superior aos demais métodos analisados e o bom desempenho do Random Linkage em ambos os conjuntos de testes realizados. Por último, ressaltamos o desempenho levemente superior do Multistart nos testes com problemas de empacotamento. 


\section{Apêndice A}

\section{Funções utilizadas nos experimentos}

As funções abaixo foram retiradas de [18]:

1. Função quartic

$$
f(x)=\frac{x_{1}^{4}}{4}-x_{1}^{2}+\frac{x_{1}}{10}+\frac{x_{2}^{2}}{10}
$$

onde $x \in \Omega=[-10,10]^{n}$ e $f\left(x^{*}\right)=-0.352386$.

2. Função six hump

$$
f(x)=\left(4-2.1 x_{1}^{2}+x_{1}^{3}\right) x_{1}^{2}+x_{1} x_{2}+\left(4 x_{2}^{2}+4\right) x_{2}^{2},
$$

onde $-3 \leq x_{1} \leq 3,-2 \leq x_{2} \leq 2$ e $f\left(x^{*}\right)=-1.0316285$.

3. Função de Shubert

$$
f(x)=\prod_{i=1}^{n} \sum_{j=1}^{5}\left(j \cos \left((j+1) x_{i}+j\right)\right),
$$

onde $n=2, x \in \Omega=[-10,10]^{n}$ e $f\left(x^{*}\right)=-186.73091$.

4. Função de Shubert Penalizada

$$
\begin{aligned}
f(x)= & \prod_{i=1}^{n} \sum_{j=1}^{5}\left(j \cos \left((j+1) x_{i}+j\right)\right)+ \\
& +\beta\left(\left(x_{1}+1.42513\right)^{2}+\left(x_{2}+0.80032\right)^{2}\right),
\end{aligned}
$$

onde $n=2, x \in \Omega=[-10,10]^{n}$ e $f\left(x^{*}\right)=-186.73091$. Para a realização dos testes foi considerado $\beta \in\{0.5,1.0\}$ referenciados como problemas A.4a e A.4b, respectivamente.

5. Função de Piccioni

$$
f(x)=10 \operatorname{sen}^{2}\left(\pi x_{1}\right)-\sum_{i=1}^{n-1}\left[\left(x_{i}-1\right)^{2}\left(1+10 \operatorname{sen}^{2}\left(\pi x_{i+1}\right)\right)\right]-\left(x_{n}-1\right)^{2},
$$

onde $x \in \Omega=[-10,10]^{n}$ e $f\left(x^{*}\right)=0.0$. Foram realizados testes para $n \in\{5,8,10,20,30\}$. 
As funções abaixo foram retiradas de [1]:

\section{Função de Levy}

$$
\begin{aligned}
f(x)= & \operatorname{sen}^{2}\left(3 \pi x_{1}\right)+\sum_{i=1}^{n-1}\left[\left(x_{i}-1\right)^{2}\left(1+\operatorname{sen}^{2}\left(3 \pi x_{i+1}\right)\right)\right]+ \\
& +\left(x_{n}-1\right)\left(1+\operatorname{sen}^{2}\left(2 \pi x_{n}\right)\right),
\end{aligned}
$$

onde $n=4$ e $x \in \Omega=[-10,10]^{n}$. A função contém 7100 mínimos locais e $f\left(x^{*}\right)=$ -11.504403 .

\section{Função de Shubert}

$$
f(x)=-\sum_{i=1}^{n} \sum_{j=1}^{5} j \operatorname{sen}\left((j+1) x_{i}+j\right),
$$

onde $n=2$ e $x \in \Omega=[-10,10]^{n}$. A função contém 400 mínimos locais e $f\left(x^{*}\right)=$ -24.062499 .

3.

$$
f(x)=\frac{1}{2} \sum_{i=1}^{n}\left(x_{i}^{4}-16 x_{i}^{2}+5 x_{i}\right),
$$

onde $n=7$ e $x \in \Omega=[-5,2]^{n}$. A função contém $2^{n}$ mínimos locais e $f\left(x^{*}\right)=-274.16316$.

\section{Função de Hansen}

$$
f(x)=\sum_{i=1}^{5}\left(i \operatorname { c o s } ( ( i - 1 ) x _ { 1 } + i ) \sum _ { j = 1 } ^ { 5 } \left(j \cos \left((j+1) x_{2}+j\right),\right.\right.
$$

onde $n=2$ e $x \in \Omega=[-10,10]^{n}$. A função contém 760 mínimos locais e $f\left(x^{*}\right)=$ -176.541793 .

5.

$$
\begin{aligned}
f(x)= & e^{\operatorname{sen}\left(50 x_{1}\right)}+\operatorname{sen}\left(60 e^{x_{2}}\right)+\operatorname{sen}\left(70 \operatorname{sen}\left(x_{1}\right)\right)+\operatorname{sen}\left(\operatorname{sen}\left(80 x_{2}\right)\right)- \\
& -\operatorname{sen}\left(10\left(x_{1}+x_{2}\right)\right)+\frac{\left(x_{1}^{2}+x_{2}^{2}\right)}{4}
\end{aligned}
$$

onde $x_{1}, x_{2} \in \Omega=[-1,1]^{n}$. A função contém 2400 mínimos locais e $f\left(x^{*}\right)=-3.306868647475$.

\section{Função de Hartman}

$$
f(x)=-\sum_{i=1}^{4} c_{i} e^{-\sum_{j=1}^{n} A_{i j}\left(x_{j}-P_{i j}\right)^{2}}
$$


onde $x \in \Omega=[0,1]^{n}$. Para $n=3 \mathrm{e}$, dados os seguintes valores

$$
A=\left(\begin{array}{ccc}
3 & 10 & 30 \\
0.1 & 10 & 35 \\
3 & 10 & 30 \\
0.1 & 10 & 35
\end{array}\right), \quad P=\left(\begin{array}{ccc}
0.3689 & 0.1170 & 0.2673 \\
0.4699 & 0.4378 & 0.7470 \\
0.1091 & 0.8732 & 0.5547 \\
0.03815 & 0.5743 & 0.8828
\end{array}\right) \text { e } \quad c=\left(\begin{array}{l}
0.1 \\
0.2 \\
0.2 \\
0.4
\end{array}\right)
$$

o valor do mínimo global corresponde a $f\left(x^{*}\right)=-3.861305797098$ [1].

\section{Função de Griewank}

$$
f(x)=\sum_{i=1}^{n} \frac{x_{i}^{2}}{4000}-\prod_{i=1}^{n} \cos \left(\frac{x_{i}}{\sqrt{i}}\right)+1
$$

onde $n=2$ e $x \in \Omega=[-500,700]^{n}$. A função contém mais de 500 mínimos locais e $f\left(x^{*}\right)=0.0$.

8.

$$
f(x)=\sum_{i=1}^{m}\left[2+2 i-\left(e^{i x_{1}}+e^{i x_{2}}\right)\right]^{2},
$$

onde $x_{1}, x_{2} \in \Omega=[-1000,5]^{n}$. Para $m=10, f\left(x^{*}\right)=124.3621823719$.

9 .

$$
f(x)=\sum_{i=1}^{11}\left[y_{i}-\frac{\left.x_{1} u_{i}\left(u_{i}+x_{2}\right)\right)}{\left(u_{i}\left(u_{i}+x_{3}\right)+x_{4}\right)}\right]^{2},
$$

onde $x \in \Omega=[-1000,1000]^{n}$. Para $n=4$ e $u_{i}=\frac{1}{b_{i}}, b=(0.25,0.5,1,2,4,6,8,10,12,14,16)^{T}$ e $y=(0.1957,0.1947,0.1735,0.1600,0.0844,0.0627,0.0456,0.0342 d 0,0.0323,0.0235,0.0246)^{T}$, o mínimo global esperado é de $f\left(x^{*}\right)=0.000307486$.

10.

$$
f(x)=\sum_{i=1}^{m}\left[\left(x_{1}+t_{i} x_{2}-e^{t_{i}}\right)^{2}+\left(x_{3}+x_{4} \operatorname{sen}\left(t_{i}\right)-\cos \left(t_{i}\right)\right)^{2}\right]^{2},
$$

onde $x \in \Omega=[-100,100]^{n}$. Para $m=20$ e $t_{i}=\frac{i}{5}$, o mínimo global esperado é de $f\left(x^{*}\right)=$ 85822.20171974 .

11.

$$
f(x)=\frac{1}{400} \sum_{i=1}^{n} x_{i}^{2}-\prod_{i=1}^{n} \cos \left(\frac{x_{i}}{\sqrt{i}}\right)+1,
$$

onde $n=5$ e $x \in \Omega=[-10,10]^{n}$ e $f\left(x^{*}\right)=0.0$.

As funções abaixo foram retiradas de [20]: 
1.

$$
f(x)=\left(x_{1}^{2}+x^{2}-11\right)^{2}+\left(x_{1}+x_{2}^{2}-7\right)^{2}
$$

onde $x \in \Omega=[-6,6]^{n}$ e $f\left(x^{*}\right)=0.0$.

2.

$$
f(x)=100\left(x_{2}-x_{1}^{2}\right)^{2}+\left(1-x_{1}\right)^{2}
$$

onde $x \in \Omega=[-2,2]^{n}$ e $f\left(x^{*}\right)=0.0$.

3.

$$
f(x)=-\sum_{i=1}^{m}\left[\left(x-a_{i}\right)^{T}\left(x-a_{i}\right)+c_{i}\right]^{-1}
$$

onde $n=4$ e $x \in \Omega=[0,10]^{n}$. Para $m=5$ o valor da função objetivo no mínimo global

\begin{tabular}{|c|c|c|c|c|c|c|c|c|c|c|c|}
\hline$i$ & \multicolumn{4}{|c|}{$a_{i}$} & $c_{i}$ & $i$ & \multicolumn{4}{|c|}{$a_{i}$} & $c_{i}$ \\
\hline 1 & 4.0 & 4.0 & 4.0 & 4.0 & 0.1 & 6 & 2.0 & 9.0 & 2.0 & 9.0 & 0.6 \\
\hline 2 & 1.0 & 1.0 & 1.0 & 1.0 & 0.2 & 7 & 5.0 & 5.0 & 3.0 & 3.0 & 0.3 \\
\hline 3 & 8.0 & 8.0 & 8.0 & 8.0 & 0.2 & 8 & 8.0 & 1.0 & 8.0 & 1.0 & 0.7 \\
\hline 4 & 6.0 & 6.0 & 6.0 & 6.0 & 0.4 & 9 & 6.0 & 2.0 & 6.0 & 2.0 & 0.5 \\
\hline 5 & 3.0 & 7.0 & 3.0 & 7.0 & 0.4 & 10 & 7.0 & 3.6 & 7.0 & 3.6 & 0.5 \\
\hline
\end{tabular}
é $f\left(x^{*}\right)=-10.1531957$ (A.19a); para $m=10, f\left(x^{*}\right)=-10.5362836$ (A.19b). Para tanto, foram utilizados os seguintes valores para os vetores $a_{i}$ e as constantes $c_{i}$ :

4.

$$
f(x)=\left(x_{1}+10 x_{2}\right)^{2}+5\left(x_{3}-x_{4}\right)^{2}+\left(x_{2}-2 x_{3}\right)^{4}+10\left(x_{1}-x_{4}\right)^{4},
$$

onde $x \in \Omega=[-3,3]^{n}$ e $f\left(x^{*}\right)=0.0$.

5.

$$
f(x)=\left(4-2.1 x_{1}^{2}+\frac{x_{1}^{4}}{3}\right) x_{1}^{2}+x_{1} x_{2}+\left(-4+4 x_{2}^{2}\right) x_{2}^{2},
$$

onde $-3 \leq x_{1} \leq 3,-2 \leq x_{2} \leq 2$ e $f\left(x^{*}\right)=-1.031628$.

6.

$$
f(x)=\left[\sum_{i=1}^{5} i \cos \left((i+1) x_{1}+i\right)\right]\left[\sum_{i=1}^{5} i \cos \left((i+1) x_{2}+i\right)\right],
$$

onde $x \in \Omega=[-10,10]^{n}$ e $f\left(x^{*}\right)=-186.7309$.

7.

$$
f(x)=\frac{1}{2} \sum_{i=1}^{n}\left(x_{i}^{4}-16 x_{i}^{2}+5 x_{i}\right)
$$

onde $x \in \Omega=[-20,20]^{n}$. Para $n=2, f\left(x^{*}\right)=-78.332331 ; n=3, f\left(x^{*}\right)=-117.4984$; $n=4, f\left(x^{*}\right)=-156.66466$. 
8.

$$
f(x)=0.5 x_{1}^{2}+0.5\left(1-\cos \left(2 x_{1}\right)\right)+x_{2}^{2},
$$

onde $x \in \Omega=[-5,5]^{n}$ e $f\left(x^{*}\right)=0.0$.

9.

$$
f(x)=10 x_{1}^{2}+x_{2}^{2}-\left(x_{1}^{2}+x_{2}^{2}\right)^{2}+10^{-1}\left(x_{1}^{2}+x_{2}^{2}\right)^{4},
$$

onde $x \in \Omega=[-5,5]^{n}$ e $f\left(x^{*}\right)=-0.407461$.

10.

$$
f(x)=10^{2} x_{1}^{2}+x_{2}^{2}-\left(x_{1}^{2}+x_{2}^{2}\right)^{2}+10^{-2}\left(x_{1}^{2}+x_{2}^{2}\right)^{4},
$$

onde $x \in \Omega=[-5,5]^{n}$ e $f\left(x^{*}\right)=-18.058697$.

11.

$$
f(x)=10^{3} x_{1}^{2}+x_{2}^{2}-\left(x_{1}^{2}+x_{2}^{2}\right)^{2}+10^{-3}\left(x_{1}^{2}+x_{2}^{2}\right)^{4},
$$

onde $x \in \Omega=[-20,20]^{n}$ e $f\left(x^{*}\right)=-227.765747$.

12.

$$
f(x)=10^{4} x_{1}^{2}+x_{2}^{2}-\left(x_{1}^{2}+x_{2}^{2}\right)^{2}+10^{-4}\left(x_{1}^{2}+x_{2}^{2}\right)^{4},
$$

onde $x \in \Omega=[-20,20]^{n}$ e $f\left(x^{*}\right)=-2429.414749$.

13.

$$
f(x)=x_{1}^{2}+x_{2}^{2}-\cos \left(18 x_{1}\right)-\cos \left(18 x_{2}\right),
$$

onde $x \in \Omega=[-5,5]^{n}$ e $f\left(x^{*}\right)=-2.0$.

14.

$$
f(x)=\left(x_{2}-\frac{5.1 x_{1}^{2}}{4 \pi^{2}}+\frac{5 x_{1}}{\pi}-6\right)^{2}+10\left(1-\frac{1}{8 \pi}\right) \cos \left(x_{1}\right)+10,
$$

onde $x \in \Omega=[-20,20]^{n}$ e $f\left(x^{*}\right)=0.397887$.

15.

$$
f(x)=-\left[\sum_{i=1}^{5} \operatorname{sen}\left((i+1) x_{1}+i\right],\right.
$$

onde $x \in \Omega=[-20,20]^{n}$ e $f\left(x^{*}\right)=-3.372897$.

16.

$$
f(x)=e^{\left(0.5\left(x_{1}^{2}+x_{2}^{2}-25\right)\right)^{2}}+\operatorname{sen}\left(4 x_{1}-3 x_{2}\right)^{4}+0.5\left(2 x_{1}+x_{2}-10\right)^{2},
$$

onde $x \in \Omega=[0,6]^{n}$ e $f\left(x^{*}\right)=1.0$.

17.

$$
f(x)=x_{1}^{6}-15 x_{1}^{4}+27 x_{1}^{2}+250,
$$

onde $x \in \Omega=[-5,5]^{n}$ e $f\left(x^{*}\right)=7.0$. 
18.

$$
\begin{aligned}
f(x)= & 100\left(x_{2}-x_{1}^{2}\right)^{2}+\left(1-x_{1}\right)^{2}+90\left(x_{4}-x_{3}^{2}\right)^{2}+\left(1-x_{3}\right)^{2}+ \\
& +10.1\left(\left(x_{2}-1\right)^{2}+\left(x_{4}-1\right)^{2}\right)+19.8\left(x_{2}-1\right)\left(x_{4}-1\right),
\end{aligned}
$$

onde $x \in \Omega=[-3,3]^{n}$ e $f\left(x^{*}\right)=0.0$.

19.

$$
f(x)=\left(1.5-x_{1}\left(1-x_{2}\right)\right)^{2}+\left(2.25-x_{1}\left(1-x_{2}^{2}\right)\right)^{2}+\left(2.625-x_{1}\left(1-x_{2}^{3}\right)\right)^{2},
$$

onde $x \in \Omega=[0,5]^{n}$ e $f\left(x^{*}\right)=0.0$.

20.

$$
f(x)=\sum_{i=1}^{10}\left(e^{-0.2 i}+2 e^{-0.4 i}-x_{1} e^{-0.2 x_{2} i}-x_{3} e^{-0.2 x_{4} i}\right)^{2},
$$

onde $x \in \Omega=[-20,7]^{n}$ e $f\left(x^{*}\right)=0.0$.

21.

$$
f(x)=\left[\sum_{i=1}^{n} \frac{x_{i}^{2}}{2^{i-1}}\right]+\left[\sum_{i=2}^{n} \frac{x_{i} x_{i-1}}{2^{i}}\right],
$$

onde $x \in \Omega=[-20,7]^{n}$ e $f\left(x^{*}\right)=0.0$. Foram realizados testes para $n \in\{10,20,30,40\}$.

22.

$$
f(x)=\sum_{i=1}^{10} x_{i}^{2}
$$

onde $x \in \Omega=[-10,7]^{n}$ e $f\left(x^{*}\right)=0.0$.

23.

$$
f(x)=\sum_{i=1}^{10}\left\lfloor x_{i}^{2}+0.5\right\rfloor^{2},
$$

onde $x \in \Omega=[-10,7]^{n}$ e $f\left(x^{*}\right)=2.5$.

24.

$$
f(x)=-20 e^{-0.2 \sqrt{0.1 \sum_{i=1}^{10} x_{i}^{2}}}-e^{0.1 \sum_{i=1}^{10} \cos \left(2 \pi x_{i}\right)}+20+e
$$

onde $x \in \Omega=[-10,20]^{n}$ e $f\left(x^{*}\right)=0.0$.

25.

$$
f(x)=\operatorname{sen}\left(x_{1}\right)+\operatorname{sen}\left(\frac{10 x_{1}}{3}\right)+\log _{10}\left(x_{1}\right)-0.84 x_{1}
$$

onde $x \in \Omega=[0.1,6]^{n}$ e $f\left(x^{*}\right)=-5.534$. 


\section{Apêndice B}

\section{Fluxogramas dos algoritmos implementados}

\section{B.1 Multistart}

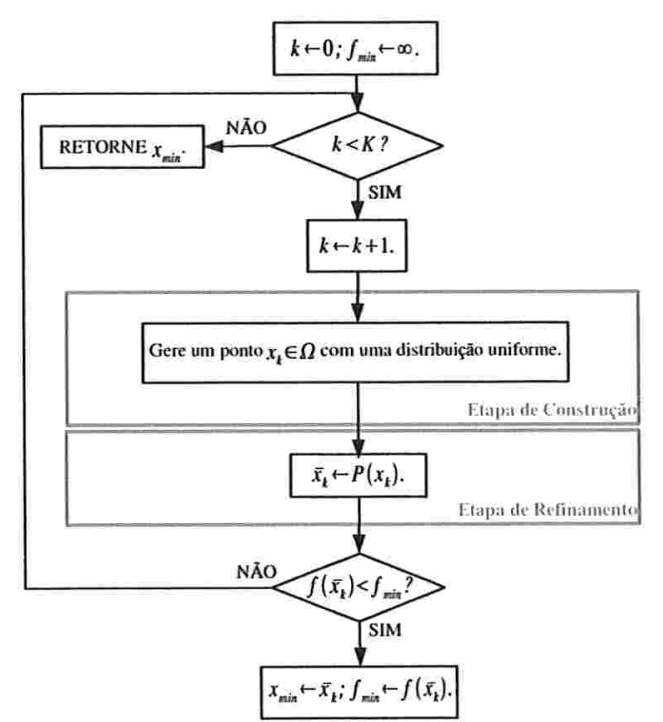

Figura B.1: Fluxograma do algoritmo Multistart. 


\section{B.2 Random Linkage}

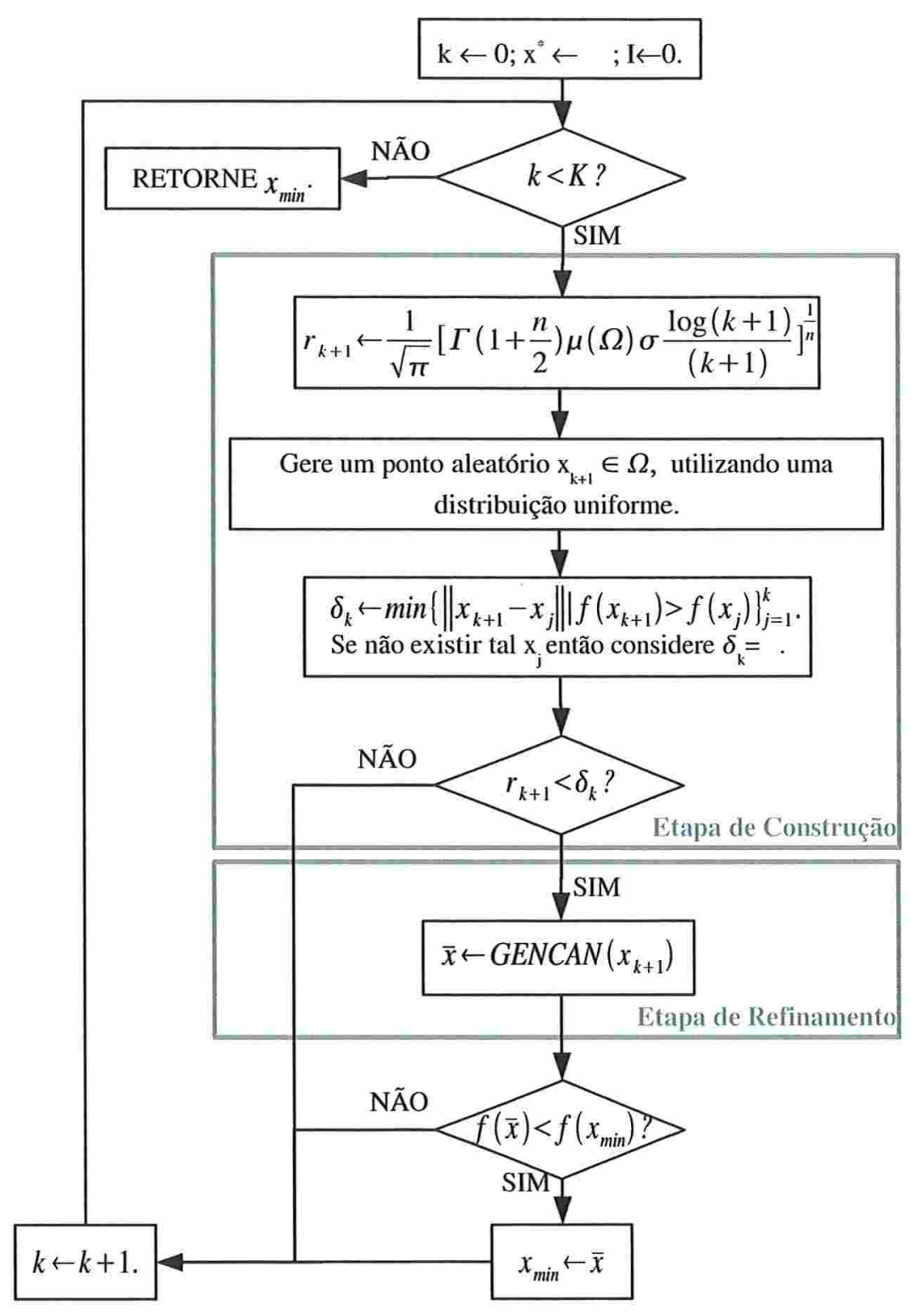

Figura B.2: Fluxograma do algoritmo Random Linkage. 


\section{B.3 Tunneling}

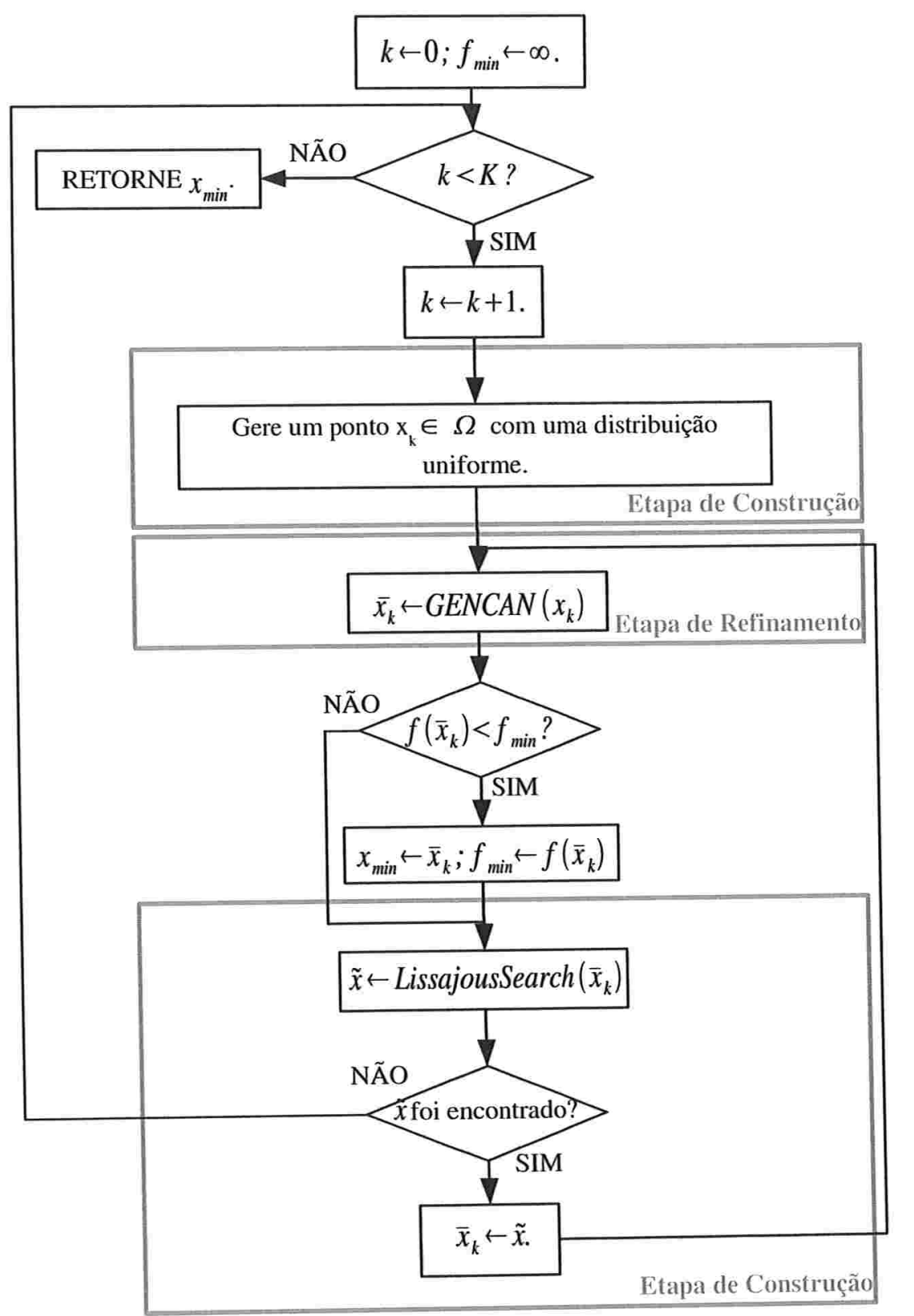

Figura B.3: Fluxograma geral do algoritmo de Tunneling utilizando a curva de Lissajous. 


\section{Procedimento LissajousSearch:}

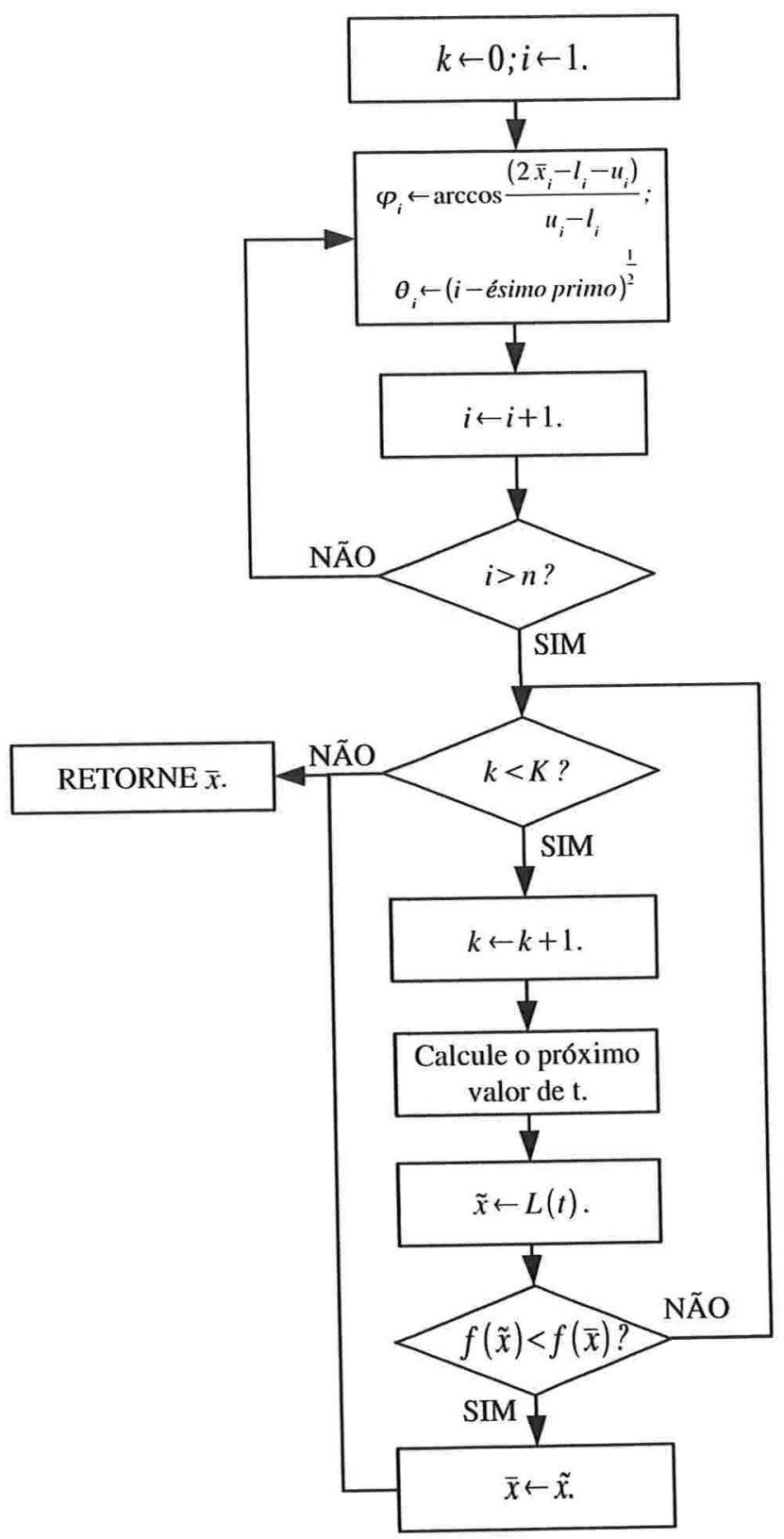

Figura B.4: Detalhe da Fase de Tunneling utilizando a curva de Lissajous. 


\section{B.4 GRASP}

\section{B.4.1 GRASP}

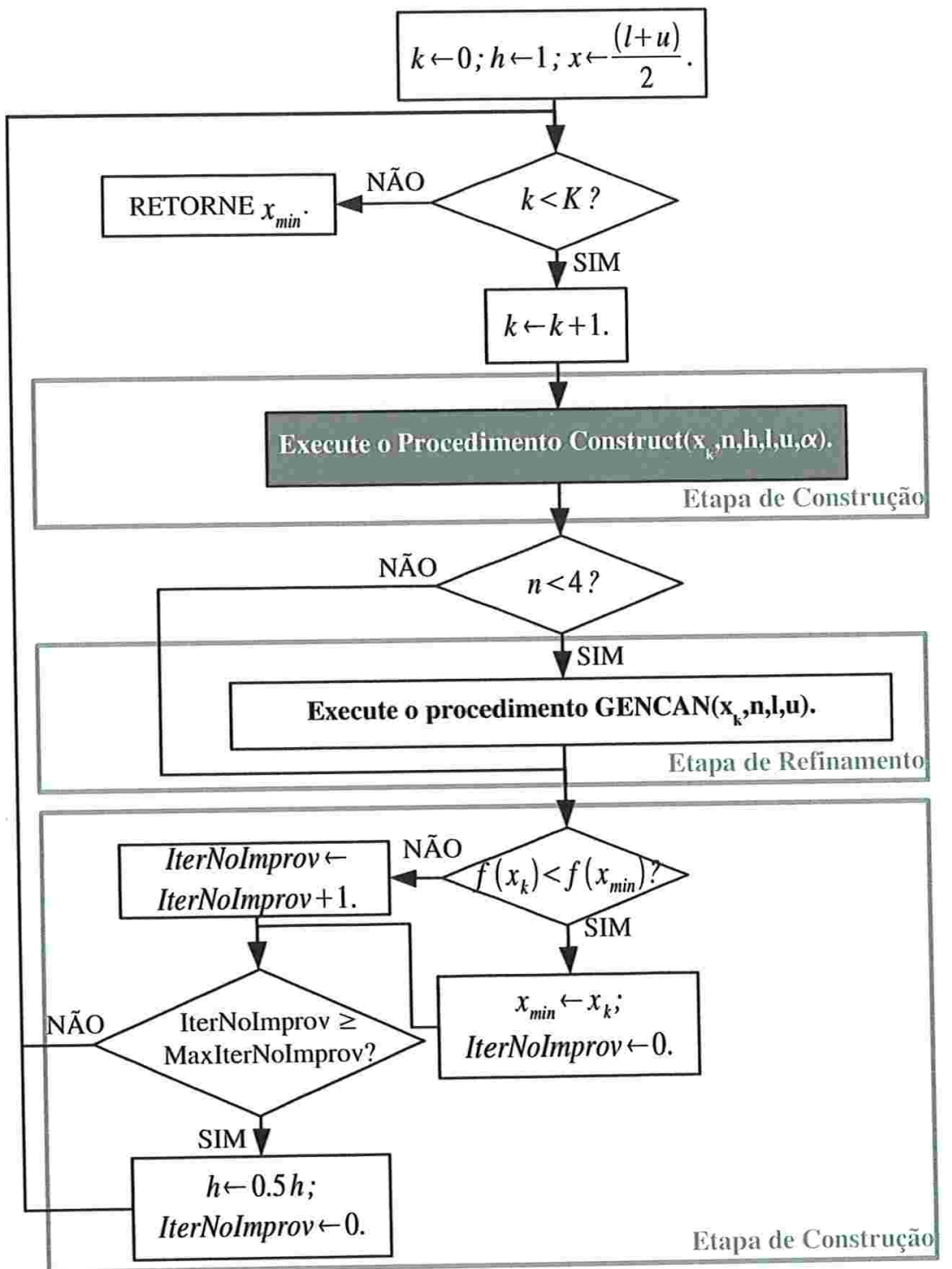

Figura B.5: Fluxograma geral do algoritmo GRASP. 


\section{Procedimento Construct:}

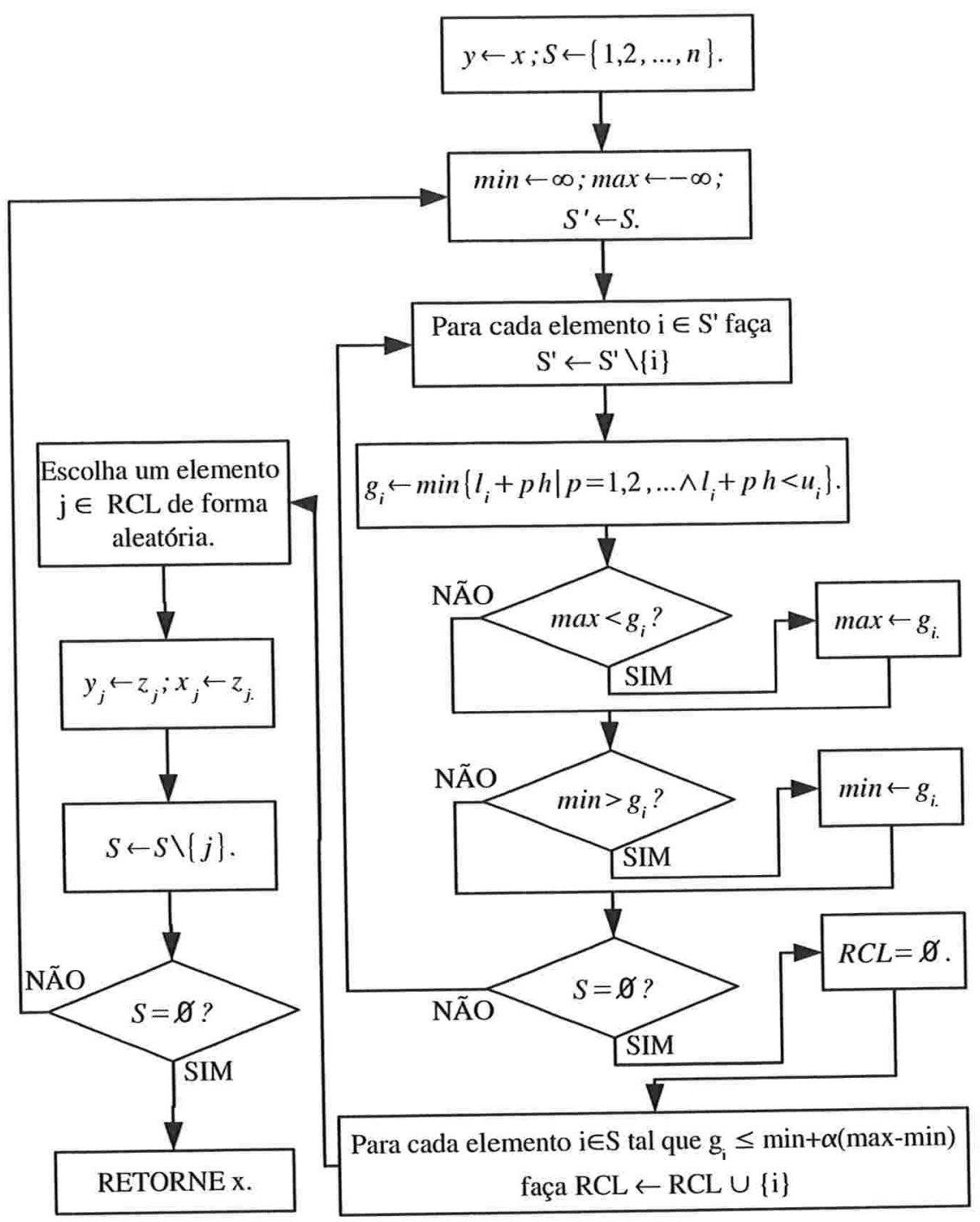

Figura B.6: Fluxograma da Etapa de Construção do GRASP. 


\section{B.4.2 GRASP contínuo}

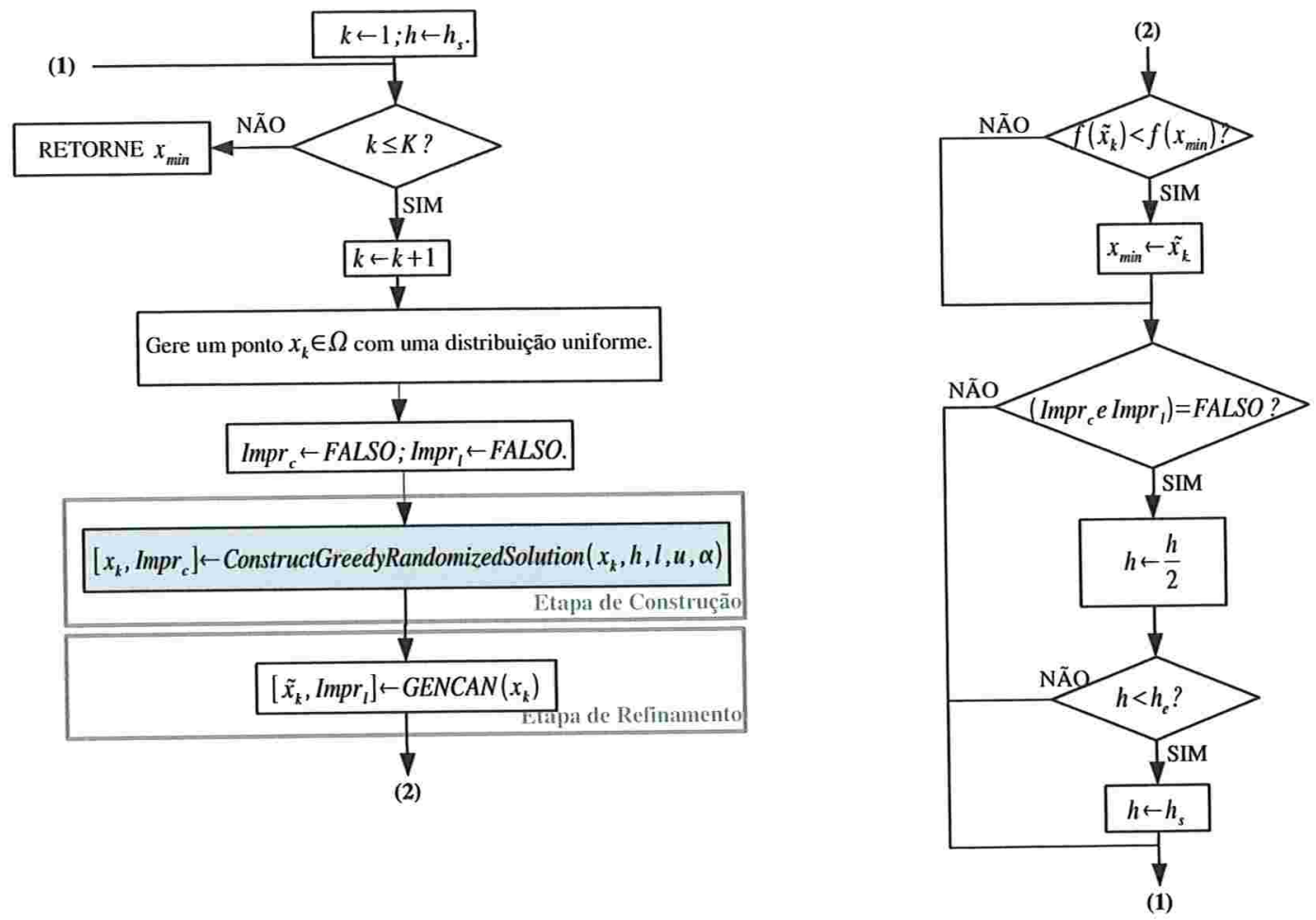

Figura B.7: Fluxograma geral do algoritmo GRASP contínuo. 
Procedimento ConstructGreedyRandomizedSolution:
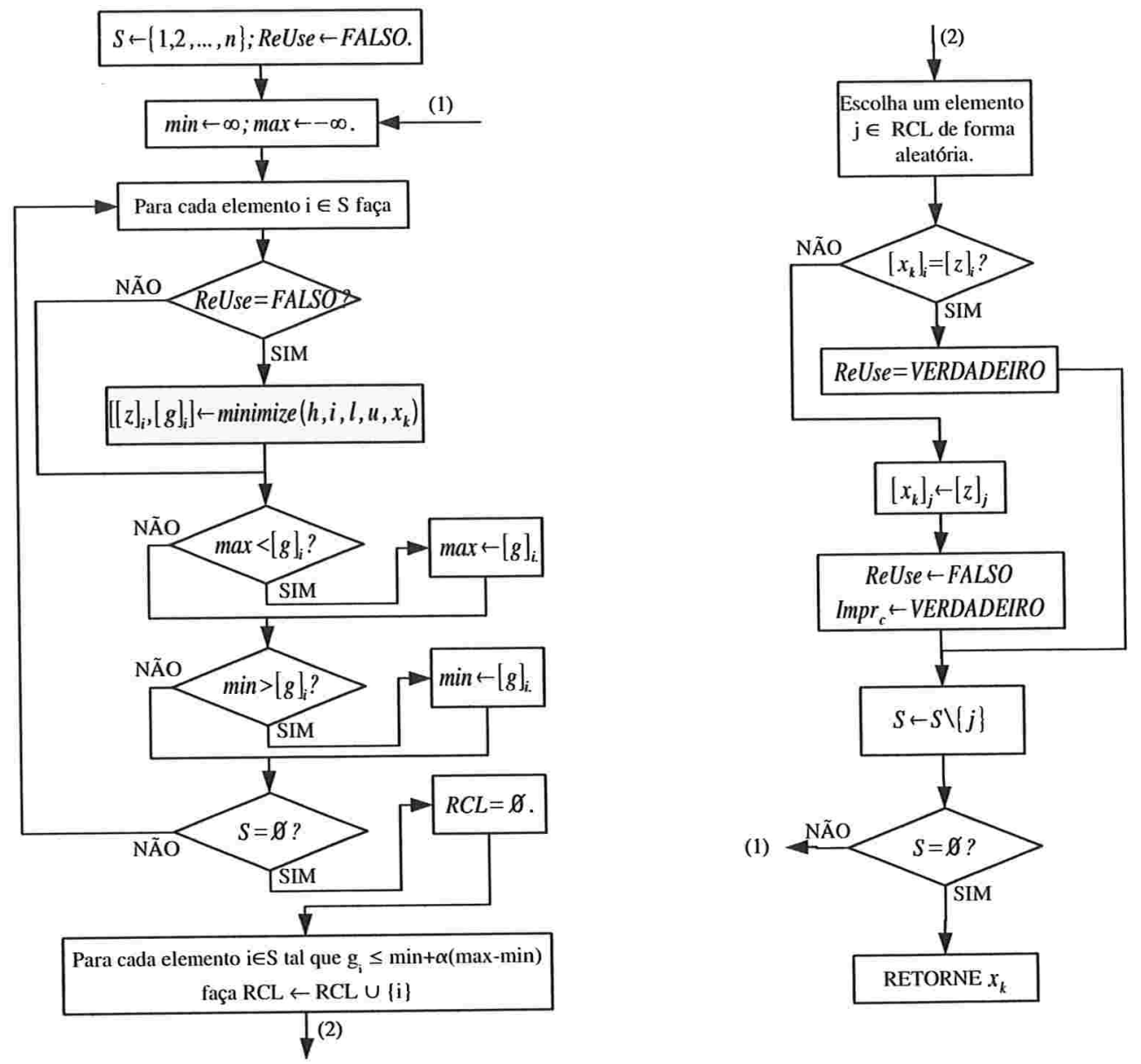

Figura B.8: Detalhes da Etapa de Construção do GRASP contínuo. 
Procedimento Minimize:

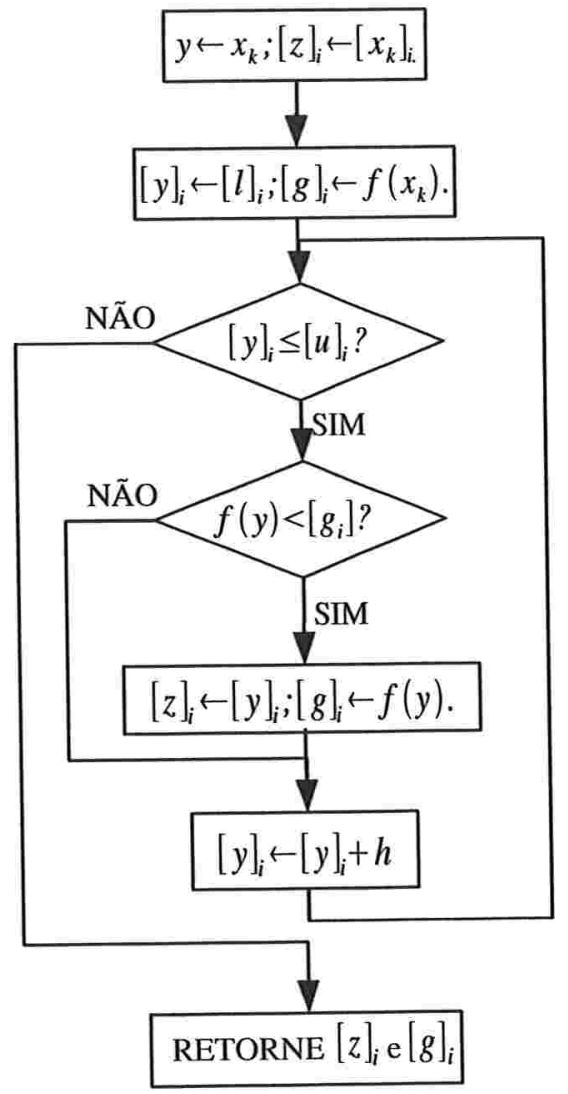

Figura B.9: Detalhes do procedimento Minimize. 


\section{Apêndice $\mathrm{C}$}

\section{Comparação alternativa dos testes realizados no Capítulo 5}

\section{C.1 Comparativo dos resultados}

De um modo geral, as soluções encontradas pelos métodos foram muito próximas. Dos 52 problemas avaliados, os métodos encontraram soluções equivalentes em 47 problemas.

Na Tabela C.1 comparamos o número de iterações utilizadas pelos métodos na resolução de cada um dos problemas. Os dados mostrados na tabela indicam a relação do número de iterações atingido por cada método com o melhor resultado encontrado para aquele problema. Desta forma, os resultados com valor igual a 1.00 refletem os métodos que encontraram a solução esperada no menor número de iterações. Os resultados da tabela mostram um desempenho muito superior das duas versões do GRASP em comparação aos demais métodos. Verificamos ainda que todos os métodos analisados tiveram desempenho médio superior ao Multistart.

Na Tabela C.2 fizemos comparação semelhante com o tempo gasto pelos métodos para encontrar a solução desejada. Visando evitar divisão por zero, consideramos os tempos encontrados pelos métodos da seguinte forma $\max \left\{0.01, t_{x^{*}}\right\}$. Os resultados mostram um desempenho superior do C-GRASP-GENCAN seguido pelo GRASP-GENCAN.

Associando os resultados das duas tabelas apresentadas identificamos, por exemplo, que o Multistart precisou, em média, de 15.6 vezes mais iterações para encontrar a mesma solução que o C-GRASP-GENCAN, mas realizou este cálculo, em média, em apenas 1.8 vezes a mais de tempo que o C-GRASP-GENCAN. Estes dados indicam que, apesar da eficiência da Etapa de Contrução do GRASP em encontrar pontos mais próximos a vizinhança de um mínimizador global, este processo consome um tempo considerável para ser realizado.

Os resultados dos testes, também apresentados de forma sintética na Tabela C.3, mostram que os algoritmos C-GRASP-GENCAN, GRASP-GENCAN, Random Linkage ( $\sigma \in\{1,2\}$ ) e Multistart foram os que apresentaram, na média, melhor desempenho para o conjunto de testes realizados. 


\begin{tabular}{|c|c|c|c|c|c|c|c|c|c|}
\hline Problema & $n$ & MST & $\mathrm{RL}(1)$ & RL(2) & RL(4) & LIS Orig & LIS Seq & GRA-GEN & C-GRA-GEN \\
\hline A.1 & 2 & 1.00 & 1.00 & 1.00 & 1.00 & 1.00 & 1.00 & 1.00 & 1.00 \\
\hline A.2 & 2 & 1.00 & 1.00 & 1.00 & 1.00 & 1.00 & 1.00 & 1.00 & 1.00 \\
\hline A.3 & 2 & 31.00 & 19.00 & 17.00 & 11.00 & 2.00 & 2.00 & 1.00 & 1.00 \\
\hline A.4a & 2 & 14.30 & 25.20 & 22.70 & 10.10 & 2.50 & 2.20 & 2.20 & 1.00 \\
\hline A.4b & 2 & 20.83 & 6.67 & 4.83 & 3.83 & 1.17 & 1.33 & 1.33 & 1.00 \\
\hline A.5 & 5 & 27.00 & 44.00 & 53.00 & 36.00 & 3.00 & 14.00 & 1.00 & 1.00 \\
\hline A.5 & 8 & 27.00 & 24.00 & 33.00 & 31.00 & 33.00 & 36.00 & 1.00 & 1.00 \\
\hline A.5 & 10 & 4.00 & 4.00 & 4.00 & 4.00 & 4.00 & 4.00 & 1.00 & 1.00 \\
\hline A.5 & 20 & 50.00 & 50.00 & 49.00 & 48.00 & 50.00 & 50.00 & 1.00 & 1.00 \\
\hline A. 5 & 30 & 6.00 & 6.00 & 5.00 & 5.00 & 6.00 & 6.00 & 1.00 & 1.00 \\
\hline A.6 & 4 & - & - & - & - & - & - & - & - \\
\hline A.7 & 2 & 18.50 & 9.25 & 7.50 & 5.75 & 1.25 & 1.00 & 1.50 & 1.25 \\
\hline A. 8 & 7 & 3.00 & 3.00 & 3.00 & 3.00 & 3.00 & 3.00 & 1.00 & 1.00 \\
\hline A.9 & 2 & 1.00 & 1.00 & 1.00 & 1.00 & 1.00 & 1.00 & 1.00 & 1.00 \\
\hline A.10 & 2 & 33.16 & 11.95 & 7.72 & 34.98 & 1.00 & 2.74 & 2.51 & 10.79 \\
\hline A.11 & 3 & 1.00 & 1.00 & 1.00 & 1.00 & 1.00 & 1.00 & 16.00 & 3.00 \\
\hline A.12 & 2 & $=$ & - & - & - & - & - & - & - \\
\hline A.13 & 2 & - & - & - & - & - & $=$ & - & - \\
\hline A.14 & 4 & - & - & - & - & - & - & - & - \\
\hline A.15 & 4 & 1.00 & 1.00 & 1.00 & 1.00 & 1.00 & 1.00 & 1.00 & 1.00 \\
\hline A.16 & 5 & 2.17 & 1.50 & 1.17 & 1.08 & 2.67 & 2.83 & 1.00 & 2.25 \\
\hline A.17 & 2 & 1.00 & 1.00 & 1.00 & 1.00 & 1.00 & 1.00 & 1.00 & 1.00 \\
\hline A. 18 & 2 & 1.00 & 1.00 & 1.00 & 1.00 & 1.00 & 1.00 & 1.00 & 1.00 \\
\hline A.19a & 4 & 3.00 & 3.00 & 3.00 & 3.00 & 3.00 & 3.00 & 1.00 & 1.00 \\
\hline A.19b & 4 & 3.00 & 1.00 & 3.00 & 3.00 & 3.00 & 3.00 & 1.00 & 1.00 \\
\hline A. 20 & 4 & 1.00 & 2.00 & 1.00 & 1.00 & 1.00 & 1.00 & 1.00 & 1.00 \\
\hline A.21 & 2 & 2.00 & 2.00 & 2.00 & 2.00 & 2.00 & 2.00 & 1.00 & 1.00 \\
\hline A. 22 & 2 & 31.00 & 19.00 & 17.00 & 11.00 & 2.00 & 2.00 & 1.00 & 1.00 \\
\hline A.23 & 2 & 1.50 & 1.50 & 1.50 & 1.50 & 1.50 & 1.00 & 1.00 & 1.00 \\
\hline A.23 & 3 & 3.00 & 3.00 & 3.00 & 3.00 & 3.00 & 2.00 & 1.00 & 1.00 \\
\hline A.23 & 4 & 20.00 & 16.00 & 17.50 & 28.50 & 20.00 & 1.50 & 1.00 & 1.00 \\
\hline A.24 & 2 & 1.00 & 1.00 & 1.00 & 1.00 & 1.00 & 1.00 & 1.00 & 1.00 \\
\hline A.25 & 2 & 1.00 & 1.00 & 1.00 & 1.00 & 1.00 & 1.00 & 1.00 & 1.00 \\
\hline A. 26 & 2 & 1.00 & 1.00 & 1.00 & 1.00 & 1.00 & 1.00 & 1.00 & 1.00 \\
\hline A.27 & 2 & 1.00 & 1.00 & 1.00 & 1.00 & 1.00 & 1.00 & 1.00 & 1.00 \\
\hline A.28 & 2 & 1.00 & 1.00 & 1.00 & 1.00 & 1.00 & 1.00 & 1.00 & 1.00 \\
\hline A.29 & 2 & 66.50 & 23.50 & 15.50 & 10.00 & 201.50 & 5.50 & 1.00 & 1.00 \\
\hline A.30 & 2 & 1.00 & 1.00 & 1.00 & 1.00 & 1.00 & 1.00 & 1.00 & 1.00 \\
\hline A.31 & 1 & 5.00 & 5.00 & 3.00 & 2.00 & 2.00 & 2.00 & 1.00 & 1.00 \\
\hline A.32 & 2 & 1.64 & 1.27 & 1.09 & 2.45 & 2.82 & 3.27 & 2.09 & 1.00 \\
\hline A.33 & 1 & 1.00 & 1.00 & 1.00 & 1.00 & 1.00 & 1.00 & 1.00 & 1.00 \\
\hline A.34 & 4 & 1.00 & 1.00 & 1.00 & 1.00 & 1.00 & 1.00 & 1.00 & 1.00 \\
\hline A.35 & 2 & 2.00 & 2.00 & 2.00 & 2.00 & 2.00 & 2.00 & 1.00 & 1.00 \\
\hline A.36 & 4 & 1.00 & 1.00 & 1.00 & 1.00 & 1.00 & 1.00 & 1.00 & 1.00 \\
\hline A.37 & 10 & 1.00 & 1.00 & 1.00 & 1.00 & 1.00 & 1.00 & 1.00 & 1.00 \\
\hline A.37 & 20 & 1.00 & 1.00 & 1.00 & 1.00 & 1.00 & 1.00 & 3.00 & 3.00 \\
\hline A.37 & 30 & 504.50 & 498.00 & 494.00 & 487.50 & 504.50 & 504.50 & 1.00 & 1.00 \\
\hline A.37 & 40 & 74.00 & 74.00 & 74.00 & 74.00 & 74.00 & 74.00 & 1.00 & 1.00 \\
\hline A.38 & 10 & 1.00 & 1.00 & 1.00 & 1.00 & 1.00 & 1.00 & 1.00 & 1.00 \\
\hline A.39 & 10 & 1.00 & 1.00 & 1.00 & 1.00 & 1.00 & 1.00 & 1.00 & 1.00 \\
\hline A.40 & 10 & - & - & - & - & - & - & - & - \\
\hline A.41 & 1 & 1.00 & 1.00 & 1.00 & 1.00 & 1.00 & 1.00 & 1.00 & 1.00 \\
\hline Média & & 20.77 & 18.66 & 18.44 & 17.99 & 20.27 & 16.02 & 1.46 & 1.33 \\
\hline
\end{tabular}

Tabela C.1: Comparativo do número de iterações realizadas por cada método para um conjunto de problemas propostos em $[18,1]$. Os números acima indicam o número de vezes de iterações que um método precisou para encontrar uma boa aproximação do minimizador global, em comparação ao método mais eficiente. 


\begin{tabular}{|c|c|c|c|c|c|c|c|c|c|}
\hline Problema & $n$ & MST & $\mathrm{RL}(1)$ & $\mathrm{RL}(2)$ & $\mathrm{RL}(4)$ & LIS Orig & LIS Seq & GRA-GEN & C-GRA-GEN \\
\hline$\overline{A \text { A.1 }}$ & 2 & 1.00 & 1.00 & 1.00 & 1.00 & 1.00 & 1.00 & 1.00 & 1.00 \\
\hline A.2 & 2 & 1.00 & 1.00 & 1.00 & 1.00 & 1.00 & 1.00 & 1.00 & 1.00 \\
\hline A.3 & 2 & 1.00 & 1.00 & 1.00 & 1.00 & 1.00 & 1.00 & 1.00 & 1.00 \\
\hline A.4a & 2 & 6.00 & 10.00 & 9.00 & 4.00 & 2.00 & 2.00 & 2.00 & 1.00 \\
\hline A. $4 \mathrm{~b}$ & 2 & 6.00 & 1.00 & 1.00 & 1.00 & 1.00 & 1.00 & 1.00 & 1.00 \\
\hline A. 5 & 5 & 2.00 & 4.00 & 5.00 & 4.00 & 1.00 & 4.00 & 1.00 & 1.00 \\
\hline A. 5 & 8 & 3.00 & 2.00 & 3.00 & 4.00 & 23.00 & 25.00 & 1.00 & 1.00 \\
\hline A. 5 & 10 & 1.00 & 1.00 & 1.00 & 1.00 & 3.00 & 3.00 & 1.00 & 1.00 \\
\hline A.5 & 20 & 15.00 & 15.00 & 14.50 & 14.00 & 53.00 & 53.00 & 2.00 & 1.00 \\
\hline A. 5 & 30 & 2.29 & 2.29 & 1.71 & 1.86 & 4.00 & 4.00 & 1.43 & 1.00 \\
\hline A.6 & 4 & - & - & - & - & - & $=$ & - & - \\
\hline A.7 & 2 & 2.00 & 1.00 & 1.00 & 1.00 & 1.00 & 1.00 & 1.00 & 1.00 \\
\hline A.8 & 7 & 1.00 & 1.00 & 1.00 & 1.00 & 1.00 & 1.00 & 1.00 & 1.00 \\
\hline A.9 & 2 & 1.00 & 1.00 & 1.00 & 1.00 & 1.00 & 1.00 & 1.00 & 1.00 \\
\hline A.10 & 2 & 9.50 & 3.50 & 2.00 & 20.17 & 1.00 & 2.67 & 1.00 & 1.83 \\
\hline A.11 & 3 & 1.00 & 1.00 & 1.00 & 1.00 & 1.00 & 1.00 & 2.00 & 1.00 \\
\hline A.12 & 2 & - & - & - & - & - & - & - & - \\
\hline A.13 & 2 & - & - & - & $=$ & - & - & $=$ & - \\
\hline A.14 & 4 & - & - & - & - & - & - & - & - \\
\hline A.15 & 4 & 1.00 & 1.00 & 1.00 & 1.00 & 1.00 & 1.00 & 2.00 & 2.00 \\
\hline A.16 & 5 & 1.00 & 1.00 & 1.00 & 1.00 & 10.00 & 11.00 & 2.00 & 1.00 \\
\hline A.17 & 2 & 1.00 & 1.00 & 1.00 & 1.00 & 1.00 & 1.00 & 1.00 & 1.00 \\
\hline A.18 & 2 & 1.00 & 1.00 & 1.00 & 1.00 & 1.00 & 1.00 & 1.00 & 1.00 \\
\hline A.19a & 4 & 1.00 & 1.00 & 1.00 & 1.00 & 1.00 & 1.00 & 1.00 & 1.00 \\
\hline A.19b & 4 & 1.00 & 1.00 & 1.00 & 1.00 & 1.00 & 1.00 & 1.00 & 1.00 \\
\hline A.20 & 4 & 1.00 & 1.00 & 1.00 & 1.00 & 1.00 & 1.00 & 1.00 & 1.00 \\
\hline A.21 & 2 & 1.00 & 1.00 & 1.00 & 1.00 & 1.00 & 1.00 & 1.00 & 1.00 \\
\hline A.22 & 2 & 1.00 & 1.00 & 1.00 & 1.00 & 1.00 & 1.00 & 1.00 & 1.00 \\
\hline A.23 & 2 & 1.00 & 1.00 & 1.00 & 1.00 & 1.00 & 1.00 & 1.00 & 1.00 \\
\hline A.23 & 3 & 1.00 & 1.00 & 1.00 & 1.00 & 1.00 & 1.00 & 1.00 & 1.00 \\
\hline A.23 & 4 & 2.00 & 2.00 & 2.00 & 4.00 & 12.00 & 1.00 & 1.00 & 1.00 \\
\hline A.24 & 2 & 1.00 & 1.00 & 1.00 & 1.00 & 1.00 & 1.00 & 1.00 & 1.00 \\
\hline A.25 & 2 & 1.00 & 1.00 & 1.00 & 1.00 & 1.00 & 1.00 & 1.00 & 1.00 \\
\hline A. 26 & 2 & 1.00 & 1.00 & 1.00 & 1.00 & 1.00 & 1.00 & 1.00 & 1.00 \\
\hline A.27 & 2 & 1.00 & 1.00 & 1.00 & 1.00 & 1.00 & 1.00 & 1.00 & 1.00 \\
\hline A.28 & 2 & 1.00 & 1.00 & 1.00 & 1.00 & 1.00 & 1.00 & 1.00 & 1.00 \\
\hline A.29 & 2 & 4.00 & 1.00 & 1.00 & 1.00 & 34.00 & 1.00 & 1.00 & 1.00 \\
\hline A.30 & 2 & 1.00 & 1.00 & 1.00 & 1.00 & 1.00 & 1.00 & 1.00 & 1.00 \\
\hline A.31 & 1 & 1.00 & 1.00 & 1.00 & 1.00 & 1.00 & 1.00 & 1.00 & 1.00 \\
\hline A.32 & 2 & 2.00 & 1.00 & 1.00 & 2.00 & 6.00 & 7.00 & 1.00 & 1.00 \\
\hline A.33 & 1 & 1.00 & 1.00 & 1.00 & 1.00 & 1.00 & 1.00 & 1.00 & 1.00 \\
\hline A. 34 & 4 & 1.00 & 1.00 & 1.00 & 1.00 & 1.00 & 1.00 & 1.00 & 1.00 \\
\hline A.35 & 2 & 1.00 & 1.00 & 1.00 & 1.00 & 1.00 & 1.00 & 1.00 & 1.00 \\
\hline A.36 & 4 & 1.00 & 1.00 & 1.00 & 1.00 & 1.00 & 1.00 & 1.00 & 1.00 \\
\hline A.37 & 10 & 1.00 & 1.00 & 1.00 & 1.00 & 1.00 & 1.00 & 1.00 & 1.00 \\
\hline A.37 & 20 & 1.00 & 1.00 & 1.00 & 1.00 & 1.00 & 1.00 & 6.00 & 5.00 \\
\hline A.37 & 30 & 10.92 & 11.23 & 11.00 & 11.23 & 138.62 & 138.62 & 1.15 & 1.00 \\
\hline A. 37 & 40 & 1.00 & 1.00 & 1.04 & 1.04 & 14.15 & 14.15 & 1.42 & 1.08 \\
\hline A.38 & 10 & 1.00 & 1.00 & 1.00 & 1.00 & 1.00 & 1.00 & 1.00 & 1.00 \\
\hline A.39 & 10 & 1.00 & 1.00 & 1.00 & 1.00 & 1.00 & 1.00 & 1.00 & 1.00 \\
\hline A.40 & 10 & - & - & - & - & - & - & - & - \\
\hline A.41 & 1 & 1.00 & 1.00 & 1.00 & 1.00 & 1.00 & 1.00 & 1.00 & 1.00 \\
\hline Média & & 2.12 & 1.89 & 1.86 & 2.20 & 7.14 & 6.39 & 1.23 & 1.13 \\
\hline
\end{tabular}

Tabela C.2: Comparativo do tempo gasto por cada método para um conjunto de problemas propostos em $[18,1]$. Os números acima indicam quantas vezes um método demorou, em comparação aos demais, para encontrar uma boa aproximação do minimizador global.

\begin{tabular}{|l|r|r|r|r|r|r|r|r|r|}
\hline Problema & $n$ & MST & RL(1) & RL(2) & RL(4) & LIS Orig & LIS Seq & GRA-GEN & C-GRA-GEN \\
\hline \hline Iterações relativas & & 20.77 & 18.66 & 18.44 & 17.99 & 20.27 & 16.02 & 1.46 & 1.33 \\
\hline Tempos relativos & & 2.12 & 1.89 & 1.86 & 2.20 & 7.14 & 6.39 & 1.23 & 1.13 \\
\hline
\end{tabular}

Tabela C.3: Sintético com as médias apresentadas nas Tabelas C.1 e C.2. 


\section{Referências Bibliográficas}

[1] I. G. Akrotirianakis, C. A. Floudas. Computational Experience with a new class of convex underestimators: box-constrained NLP problems, Journal of Global Optimization 29, pp. 249-264, 2004.

[2] R. Andreani, E. G. Birgin, J. M. Martínez, M. L. Schuverdt. Augmented Lagrangian methods under the Constant Positive Linear Dependence constraint qualification, por aparecer em Mathematical Programming, 2006. (DOI: 10.1007/s10107-006-0077-1)

[3] R. Andreani, E. G. Birgin, J. M. Martínez, M. L. Schuverdt. On Augmented Lagrangian methods with general lower-level constraints, Technical Report MCDO-050304, Department of Applied Mathematics, UNICAMP, Brazil, 2005.

[4] G. de Barra. Introduction to Measure Theory, Van Nostrand Reinhold Company Ltd, Great Britain, 1974.

[5] E. G. Birgin, J. M. Martínez. Large-scale active-set box-constrained optimization method with spectral projected gradients, Computational Optimization and Applications 23, pp. 101-125, 2002.

[6] E. G. Birgin, J. M. Martínez, D. P. Ronconi, Optimizing the packing of cylinders into a rectangular container: a nonlinear approach, European Journal of Operational Research 160 , pp. 19-33, 2005.

[7] C. G. E. Boender, H. E. Romeijn. Stochastic methods em Handbook of Global Optimization, pp. 829-869, Kluwer Academic Publishers, Holanda, 1995.

[8] V. Chvátal. A greedy heuristic for the set convering problem, Mathematics of Operations Research 4, pp. 233-235, 1979.

[9] L. C. W. Dixon, E. Spedicato, G. P. Szegö. Nonlinear optimisation, theory and algorithms, Birkhauser Boston, 1980.

[10] E. D. Dolan, J. J. Moré. Benchmarking optimization software with performance profiles, Mathematical Programming 91, pp. 201-213, 2002.

[11] T. A. Feo, M. G. C. Resende. A probabilistic heuristic for a computationally difficult set covering problem, Operations Research Letters 8, pp. 67-71, 1989. 
[12] T. A. Feo, M. G. C. Resende. Greedy randomized adaptive search procedures, Journal of Global Optimization 6, pp.109-133, 1995.

[13] H. L. Guidorizzi. Um curso de Cálculo - Volume 2 (5a. edição), LTC Editora, Brasil, 2004.

[14] M. J. Hirsch, C. N. Meneses, P. M. Pardalos, M. G. C. Resende. Global optimization by continuous GRASP, Technical Report TD-6MPUV9. AT\&T Labs Research, 2006.

[15] M. J. Hirsch, P. M. Pardalos, M. G. C. Resende. Speeding up continuous GRASP, Technical Report TD-6U2P2H, AT\&T Shannon Laboratory, 2006.

[16] A. V. Levy, S. Gomez. The Tunneling method applied to global optimization, em Numerical Optimization 1984 (Ed. P. T. Bogus), Philadelphia, SIAM, pp. 213-244, 1985.

[17] A. V. Levy, A. Montalvo. The Tunneling algorithm for the global minimization of functions, SIAM Journal on Scientific Computing 6, pp. 15-29, 1985.

[18] M. Locatelli, F. Schoen. Numerical experience with Random Linkage algorithms for global optimisation, Technical Report 15-98, Dip. di Sistemi e Informatica, Università di Firenze, Italy, 1998.

[19] M. Locatelli, F. Schoen. Random Linkage: A family of acceptance/rejection algorithms for global optimization, Mathematical Programming 85, pp. 379-396, 1999.

[20] C. N. Meneses, P. M. Pardalos, M. G. C. Resende. GRASP for nonlinear optimization, Technical Report TD-6DUTRG, ATET Labs Research, 2005.

[21] P. M. Pardalos, Fabio Schoen. Recent advances and ternds in global optimization: Deterministic and stochastic methods, Proceedings of the Sixth International Conference on Foundations of Computer-Aided Process Design, Austin, TX, pp. 119-131, 2004.

[22] M. G. C. Resende, C. C. Ribeiro. Greedy randomized adaptive search procedures em Handbook of Metaheuristics (eds. F. Glover e G. Kochenberger), Kluwer, pp. 219-250, 2002.

[23] M. Salvatierra. Otimização global usando trajetórias densas e aplicações, tese de Doutorado, Departamento de Matemática Aplicada, IMECC, UNICAMP, 2005.

[24] A. H. G. Rinnooy-Kan, G. Timmer. Stochastic global optimization methods. part I: Clustering methods, Mathematical Programming 39, pp. 27-56, 1987.

[25] A. H. G. Rinnooy-Kan, G. Timmer. Stochastic global optimization methods. part II: Multi level methods, Mathematical Programming 39, pp. 57-78, 1987. 